\title{
Mapping Neutralizing and Immunodominant Sites on the SARS-CoV-2 Spike Receptor-Binding Domain by Structure-Guided High-Resolution Serology
}

\section{Graphical Abstract}

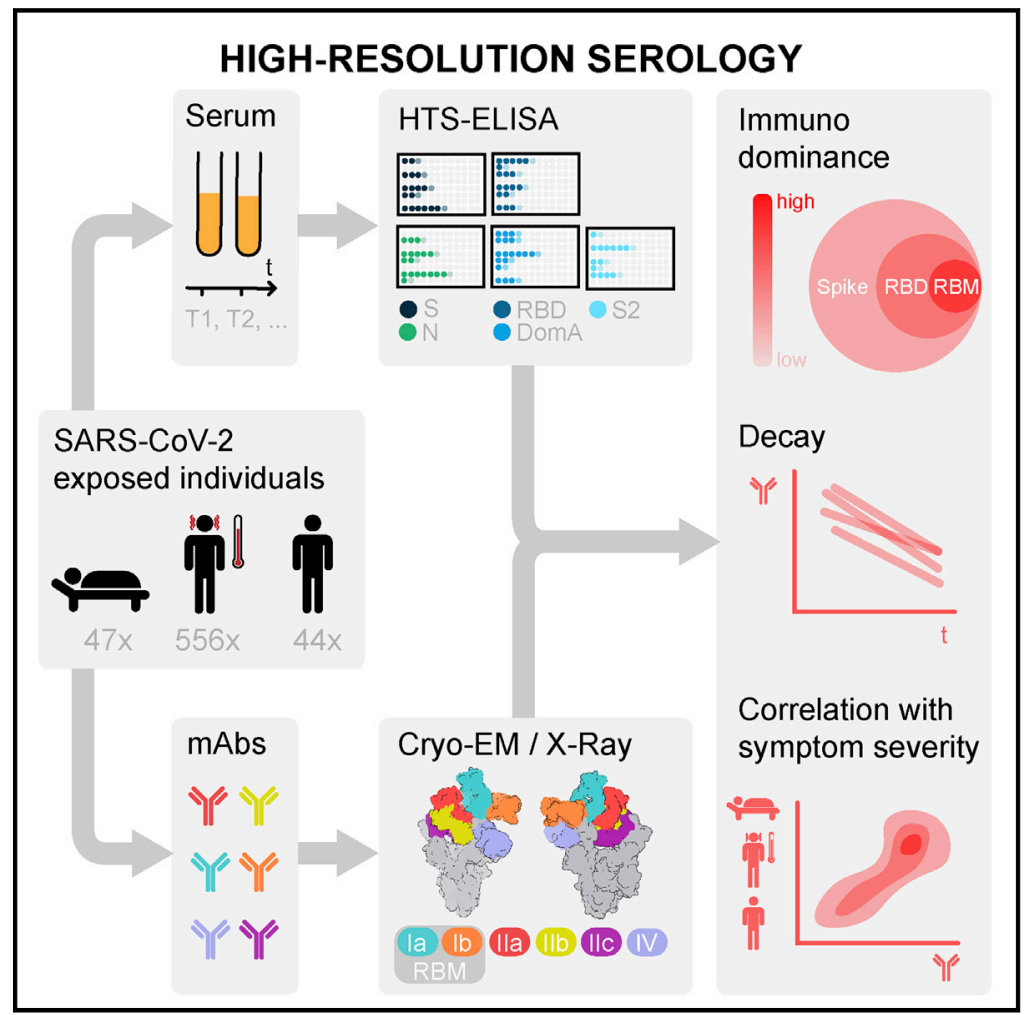

\section{Highlights}

- SARS-CoV-2 RBD is immunodominant and accounts for $90 \%$ of serum neutralizing activity

- RBD antibodies decline with a half-life of $\sim 50$ days, but their avidity increases

- Structural definition of a SARS-CoV-2 RBD antigenic map using monoclonal antibodies

- ACE2-binding site dominates SARS-CoV-2 polyclonal neutralizing antibody responses

\section{Authors}

Luca Piccoli, Young-Jun Park, M. Alejandra Tortorici, ..., Antonio Lanzavecchia, Davide Corti, David Veesler

\section{Correspondence}

dcorti@vir.bio (D.C.), dveesler@uw.edu (D.V.)

\section{In Brief}

Serological analyses of $\sim 650$ SARS-CoV2 -exposed individuals show that $90 \%$ of the serum or plasma neutralizing activity targets the virus receptor-binding domain, with structural insights revealing how distinct types of neutralizing antibodies targeting the ACE2-binding site dominate the immune response against SARS-CoV-2 spike. 


\title{
Mapping Neutralizing and Immunodominant Sites on the SARS-CoV-2 Spike Receptor-Binding Domain by Structure-Guided High-Resolution Serology
}

\author{
Luca Piccoli,, 19 Young-Jun Park, ${ }^{2,19}$ M. Alejandra Tortorici, $2,3,19$ Nadine Czudnochowski, ${ }^{4,19}$ Alexandra C. Walls, ${ }^{2}$ \\ Martina Beltramello, ${ }^{1}$ Chiara Silacci-Fregni, ${ }^{1}$ Dora Pinto, ${ }^{1}$ Laura E. Rosen, ${ }^{4}$ John E. Bowen, ${ }^{2}$ Oliver J. Acton, ${ }^{2}$ \\ Stefano Jaconi, ${ }^{1}$ Barbara Guarino, ${ }^{1}$ Andrea Minola, ${ }^{1}$ Fabrizia Zatta, ${ }^{1}$ Nicole Sprugasci, ${ }^{1}$ Jessica Bassi, ${ }^{1}$ Alessia Peter, ${ }^{1}$ \\ Anna De Marco, ${ }^{1}$ Jay C. Nix, ${ }^{5}$ Federico Mele, ${ }^{6}$ Sandra Jovic, ${ }^{6}$ Blanca Fernandez Rodriguez, ${ }^{6}$ Sneha V. Gupta, ${ }^{4}$ Feng Jin, ${ }^{4}$ \\ Giovanni Piumatti, ${ }^{7,8}$ Giorgia Lo Presti, ${ }^{9}$ Alessandra Franzetti Pellanda, ${ }^{9}$ Maira Biggiogero, ${ }^{9}$ Maciej Tarkowski, ${ }^{10}$ \\ Matteo S. Pizzuto, ${ }^{1}$ Elisabetta Cameroni, ${ }^{1}$ Colin Havenar-Daughton, ${ }^{4}$ Megan Smithey, ${ }^{4}$ David Hong, ${ }^{4}$ Valentino Lepori, ${ }^{11}$ \\ Emiliano Albanese, ${ }^{12}$ Alessandro Ceschi, $8,13,14$ Enos Bernasconi, ${ }^{15}$ Luigia Elzi, ${ }^{16}$ Paolo Ferrari, ${ }^{17,}{ }^{18}$ Christian Garzoni, ${ }^{9}$ \\ Agostino Riva, ${ }^{10}$ Gyorgy Snell, ${ }^{4}$ Federica Sallusto, ${ }^{6}$ Katja Fink, ${ }^{1}$ Herbert W. Virgin, ${ }^{4}$ Antonio Lanzavecchia, ${ }^{1}$ \\ Davide Corti, ${ }^{1, *}$ and David Veesler $2,20, *$ \\ ${ }^{1}$ Humabs BioMed SA, Vir Biotechnology, 6500 Bellinzona, Switzerland \\ 2Department of Biochemistry, University of Washington, Seattle, WA 98195, USA \\ ${ }^{3}$ Institut Pasteur and CNRS UMR 3569, Unité de Virologie Structurale, 75015 Paris, France \\ ${ }^{4}$ Vir Biotechnology, San Francisco, CA 94158, USA \\ ${ }^{5}$ Molecular Biology Consortium, Advanced Light Source, Lawrence Berkeley National Laboratory, Berkeley, CA 94720, USA \\ 6Institute for Research in Biomedicine, Università della Svizzera italiana, 6500 Bellinzona, Switzerland \\ 7Division of Primary Care, Geneva University Hospitals, 1205 Geneva, Switzerland \\ ${ }^{8}$ Faculty of Biomedical Sciences, Università della Svizzera italiana, 6900 Lugano, Switzerland \\ ${ }^{9} \mathrm{Clinic}$ of Internal Medicine and Infectious Diseases, Clinica Luganese Moncucco, 6900 Lugano, Switzerland \\ 10III Division of Infectious Diseases, ASST Fatebenefratelli Sacco, Luigi Sacco Hospital, 20157 Milan, Italy \\ ${ }^{11}$ Independent Physician, 6500 Bellinzona, Switzerland \\ ${ }^{12}$ Institute of Public Health, Università della Svizzera italiana, 6900 Lugano, Switzerland \\ ${ }^{13}$ Division of Clinical Pharmacology and Toxicology, Institute of Pharmacological Sciences of Southern Switzerland, Ente Ospedaliero \\ Cantonale, 6900 Lugano, Switzerland \\ ${ }^{14}$ Department of Clinical Pharmacology and Toxicology, University Hospital Zurich, 8091 Zurich, Switzerland \\ ${ }^{15}$ Division of Infectious Diseases, Ente Ospedaliero Cantonale, Ospedale Civico and Ospedale Italiano, 6900 Lugano, Switzerland \\ ${ }^{16}$ Division of Infectious Diseases, Ente Ospedaliero Cantonale, Ospedale Regionale Bellinzona e Valli and Ospedale Regionale, 6600 Locarno, \\ Switzerland \\ 17Department of Nephrology, Ospedale Civico Lugano, Ente Ospedaliero Cantonale, 6900 Lugano, Switzerland \\ ${ }^{18}$ Prince of Wales Hospital Clinical School, University of New South Wales, Sydney, NSW 2052, Australia \\ ${ }^{19}$ These authors contributed equally \\ 20 Lead Contact \\ *Correspondence: dcorti@vir.bio (D.C.), dveesler@uw.edu (D.V.) \\ https://doi.org/10.1016/j.cell.2020.09.037
}

\section{SUMMARY}

Analysis of the specificity and kinetics of neutralizing antibodies (nAbs) elicited by SARS-CoV-2 infection is crucial for understanding immune protection and identifying targets for vaccine design. In a cohort of 647 SARS-CoV-2-infected subjects, we found that both the magnitude of Ab responses to SARS-CoV-2 spike (S) and nucleoprotein and nAb titers correlate with clinical scores. The receptor-binding domain (RBD) is immunodominant and the target of $90 \%$ of the neutralizing activity present in SARS-CoV-2 immune sera. Whereas overall RBD-specific serum IgG titers waned with a half-life of 49 days, nAb titers and avidity increased over time for some individuals, consistent with affinity maturation. We structurally defined an RBD antigenic map and serologically quantified serum Abs specific for distinct RBD epitopes leading to the identification of two major receptor-binding motif antigenic sites. Our results explain the immunodominance of the receptor-binding motif and will guide the design of COVID-19 vaccines and therapeutics.

\section{INTRODUCTION}

Coronavirus disease 2019 (COVID-19) is caused by infection with the severe acute respiratory syndrome coronavirus 2
(SARS-CoV-2), which emerged at the end of 2019 in Wuhan, China. SARS-CoV-2 has rapidly spread worldwide and caused the ongoing COVID-19 pandemic with more than 23 million infections and over 800,000 fatalities. SARS-CoV-2 is related to 
SARS-CoV (sarbecovirus subgenus) and is more genetically distinct from the other two milder endemic human HKU-1 and OC43 viruses (embecovirus subgenus), which belong to the same $\beta$-coronavirus genus.

The ORF1a/b region of the $30 \mathrm{~kb}$ viral RNA genome encodes for most of the non-structural proteins, whereas the rest of the genome encodes for accessory proteins and four essential structural proteins, including the spike (S), envelope (E), membrane $(\mathrm{M})$, and nucleocapsid $(\mathrm{N})$ proteins. The $\mathrm{N}$ protein is the most abundant protein in virions, and its primary role is to package the viral RNA genome into a ribonucleoprotein complex. SARS-CoV-2 $\mathrm{N}$ shares limited amino acid sequence identity with OC43 and HKU-1 ( 35\%). Although coronavirus infections induce a strong antibody $(\mathrm{Ab})$ response against $\mathrm{N}$, these Abs are not neutralizing.

Similar to other coronaviruses, SARS-CoV-2 entry into host cells is mediated by the transmembrane spike (S) glycoprotein, which forms prominent homotrimers protruding from the viral surface (Ke et al., 2020; Tortorici and Veesler, 2019; Turoňová et al., 2020; Walls et al., 2016a; 2017). S comprises (1) an S $_{1}$ subunit, which recognizes host cell receptors (and is divided into $A$, $B, C$, and D domains), and (2) an $S_{2}$ subunit that promotes fusion of the viral and cellular membranes to initiate infection (Walls et al., 2020; Wrapp et al., 2020). In addition to the canonical $\mathrm{S}_{2}{ }^{\prime}$ cleavage site, SARS-CoV-2 S harbors a polybasic furin cleavage site at the $S_{1} / S_{2}$ boundary between the two $S$ functional subunits, which is unique within the sarbecovirus subgenus and key for infectivity and virulence (Hoffmann et al., 2020a; 2020b; Lau et al., 2020; Millet and Whittaker, 2015; Walls et al., 2020). The SARS-CoV-2 S domain B (so-called receptor-binding domain or RBD) binds to angiotensin-converting enzyme 2 (ACE2), which serves as an entry receptor (Hoffmann et al., 2020b; Letko et al., 2020; Walls et al., 2020; Wrapp et al., 2020; Zhou et al., 2020a), and the molecular details of attachment have been recently unveiled (Lan et al., 2020; Shang et al., 2020; Wang et al., 2020b; Yan et al., 2020).

As the coronavirus $\mathrm{S}$ glycoprotein mediates entry into host cells and is prominently exposed at the viral surface, it is the main target of neutralizing Abs (nAbs) and has been a focus of therapeutic and vaccine design efforts (Tortorici and Veesler, 2019). The protective role of Abs against coronaviruses was demonstrated 30 years ago in humans challenged with the $\alpha$-coronavirus 229E; this protection was transient when subjects were re-challenged a year later (Callow, 1985; Callow et al., 1990). Patients with severe Middle East respiratory syndrome (MERS)-associated pneumonia had persistent Ab responses detected for $>18$ months after infection, whereas asymptomatic patients or patients with mild disease had no detectable MERS-CoV-specific Ab response (Alshukairi et al., 2016; Drosten et al., 2014). Although SARS-CoV-specific immunoglobulin Gs (IgGs) have been shown to persist for at least 12 years in healthcare workers (Guo et al., 2020), several studies of SARSCoV- or MERS-CoV-infected individuals documented rapid waning of and/or the presence of limited neutralizing Ab titers over time (Cao et al., 2007; Drosten et al., 2014). Similarly, a recent serological study monitoring healthy subjects for 35 years found that reinfections by one of the four seasonal human coronaviruses (OC43, HKU-1, 229E, and NL63) occurred frequently
12 months after a primary infection, and substantial reductions in $A b$ titers were observed as soon as 6 months post-infection (Edridge et al., 2020). Several recent studies have documented the development of $\lg \mathrm{G}, \lg \mathrm{A}$, and $\lg \mathrm{M}$ against $\mathrm{S}$ and $\mathrm{N}$ within 2 weeks after onset of symptoms in SARS-CoV-2-infected individuals and analyzed the kinetics in the early convalescent phase (Long et al., 2020; Luchsinger et al., 2020; Prévost et al., 2020; Robbiani et al., 2020). Another recent study analyzed the kinetics of serum nAbs in SARS-CoV-2-infected individuals in the UK and demonstrated a constant decline of this response in the majority of individuals (Seow et al., 2020).

Although deployment of a vaccine will be the most cost-effective approach to curtail the COVID-19 pandemic and prevent the re-emergence of SARS-CoV-2, immediate solutions are needed. Recent small-scale studies suggest that treatment of COVID-19 patients with convalescent plasma improved clinical outcomes and decreased viral loads (Duan et al., 2020; Li et al., 2020a; Liu et al., 2020b; Shen et al., 2020), highlighting the importance of nAbs against SARS-CoV-2. Furthermore, passive transfer of potently neutralizing human monoclonal Abs (mAbs) protected animals challenged with SARS-CoV-2 (Alsoussi et al., 2020; Wu et al., 2020), various SARS-CoV isolates (Menachery et al., 2016; Rockx et al., 2008; Traggiai et al., 2004), and MERS-CoV (Corti et al., 2015; de Wit et al., 2019; Johnson et al., 2016). Prophylactic or therapeutic use of neutralizing mAbs could thus help control SARS-CoV-2 transmission by providing immediate protection. We recently described the mAb S309, which was isolated from the memory $B$ cells of a SARS survivor (10 years after infection) and neutralized both SARS-CoV and SARS-CoV-2 by recognizing a conserved RBD epitope (Pinto et al., 2020). Furthermore, recent reports have described the isolation of mAbs from SARS-CoV-2-infected individuals, and a few of them were characterized structurally (Barnes et al., 2020; Baum et al., 2020; Brouwer et al., 2020; Chi et al., 2020; Rogers et al., 2020; Seydoux et al., 2020; Wang et al., 2020a; Wu et al., 2020). However, the kinetics of elicitation and waning of SARSCoV-2 Abs and a comprehensive understanding of the epitopes targeted by neutralizing mAbs upon natural SARS-CoV-2 infection remain largely elusive.

We describe here the specificity and kinetics of $A b$ responses to the SARS-CoV-2 N and S proteins based on analysis of 647 plasma and serum samples from hospitalized, symptomatic, and asymptomatic COVID-19 patients. Hospitalized individuals developed higher titers of serum IgG and detectable levels of IgA compared to non-hospitalized or asymptomatic subjects in which we observed a highly heterogeneous response. We found that more than $90 \%$ of the SARS-CoV-2 neutralizing humoral responses are accounted for by RBD-directed Abs and obtained a map of the major RBD antigenic sites through structural studies of $m A b s$ derived from SARS-CoV-2 and SARS-CoV immune donors. Development of an RBD blockade-of-binding assay enabled quantitative evaluation of $A b$ responses against each RBD antigenic site in SARS-CoV-2-exposed individuals and identified two sites targeted by most serum Abs. These results provide a structural framework to understand the humoral immune response against SARS-CoV-2 and will guide future serology studies as well as vaccine and therapeutic design strategies. 

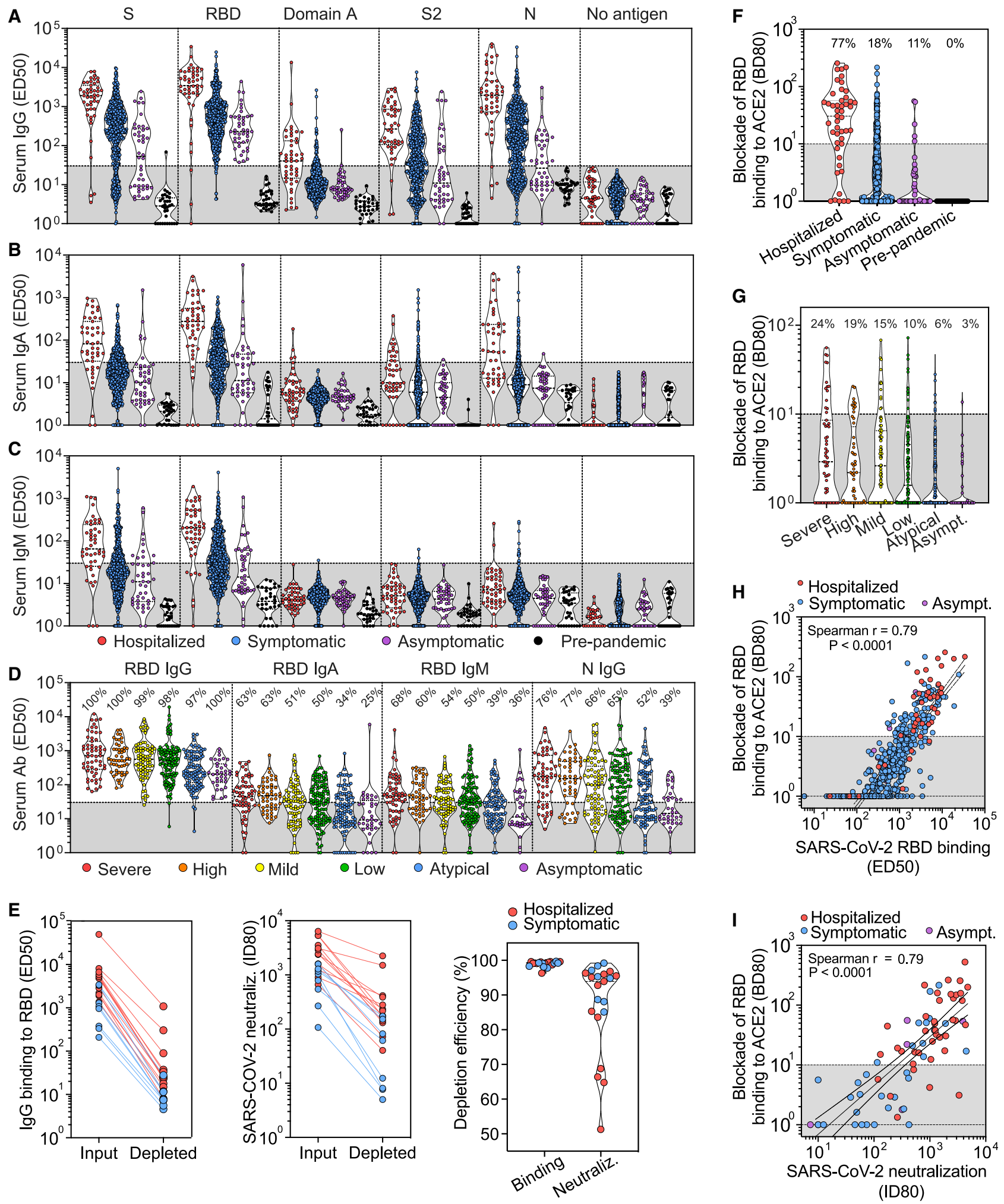


\section{RESULTS}

The IgG, IgA, and IgM Responses to SARS-CoV-2 Infection

We analyzed plasma or serum samples collected between March and July 2020 from 647 SARS-CoV-2-infected individuals, as determined by PCR $(n=271)$ or by diagnosis based on signs and symptoms ( $n=376$ ). A total of 1,078 samples, including multiple time points, were collected from five different cohorts in Italy, Switzerland, and the United States, which comprised 47 hospitalized, 556 symptomatic, and 44 asymptomatic individuals, as well as 32 pre-pandemic healthy donors (Figures S1A-S1C). For each sample, we evaluated IgG, IgA, and IgM binding titers to the SARS-CoV-2 prefusion-stabilized S ectodomain trimer (Walls et al., 2020), the RBD, domain A (residues 14-302), the $S_{2}$ subunit (residues 685-1211), and the $N$ protein by ELISA.

The IgG responses were on average 1-2 orders of magnitude higher in hospitalized relative to non-hospitalized individuals and varied across SARS-CoV-2 antigens and among subjects (Figures $1 \mathrm{~A}$ and S2A; Data S1). Males had higher Ab titers than females, although no correlation with age was observed (Data S2). Levels of SARS-CoV-2 S- and N-specific IgG correlated within each individual ( $p$ value $<0.0001$ ) (Figure S2D). SARSCoV-2 RBD-specific Abs dominated IgG responses whereas much lower titers were observed to the $S_{2}$ subunit or domain $A$ (Figure 1A). These findings might be related to the more extensive $\mathrm{N}$-glycan shielding of domain $\mathrm{A}$ and the $\mathrm{S}_{2}$ subunit, which respectively harbor 8 and 9 oligosaccharides, relative to the RBD that only possesses $2 \mathrm{~N}$-linked glycans (Walls et al., 2020; Watanabe et al., 2020). We also found that the majority of samples contained IgG cross-reactive to the SARS-CoV prefusion-stabilized S ectodomain (Walls et al., 2019) and RBD with 3-fold and 15-fold lower binding titers than those for the corresponding SARS-CoV-2 antigens, respectively (Figures $\mathrm{S} 2 \mathrm{E}-\mathrm{S} 2 \mathrm{G})$. The observed Ab cross-reactivity between these two viruses are consistent with the $76 \%$ sequence identity shared between the two $S$ glycoprotein ectodomains and recent findings (Barnes et al., 2020).

IgA responses to SARS-CoV-2 $S$ and $N$ were detected almost exclusively in hospitalized patients (Figures 1B and S2B; Data S1 and $\mathrm{S} 2$ ), whereas IgM responses were limited to $S$ and undetectable for N (Figures 1C and S2C; Data S1 and S2). In addition, we observed that SARS-CoV-2 S- and RBD-specific IgM were detectable up to 60 days after symptom onset, suggesting that detection of IgM antibodies is not associated with an ongoing or recent infection (Figure S2C). Finally, in the 459 individuals of the Ticino healthcare workers cohort, for which a symptom score was available, we observed that SARS-CoV-2 RBD-specific IgG, IgA, and IgM and SARS-CoV-2 N-specific IgG binding titers were proportional to the severity of symptoms (Figure 1D; Data S2). This serological analysis indicates that $A b$ responses varied among different individuals and among groups, with binding titers proportional to the severity of symptoms, possibly due to a prolonged exposure to large amounts of viral antigens during the course of viral disease.

\section{Function of Abs in Blocking S Interactions with ACE2}

We next determined if the RBD is the primary target of neutralizing Abs in COVID-19 convalescent plasma by measuring neutralizing titers before and after $A b$ depletion using RBDcoated beads. We found that an almost complete depletion of RBD-specific Abs from 21 plasma samples reduced SARSCoV-2 neutralizing titers by $\sim 90 \%$ on average (Figure 1E; Data S2). We then evaluated whether RBD-specific Abs in patient serum or plasma samples inhibit binding of the SARS-CoV-2 RBD to ACE2. Although $77 \%$ of hospitalized individuals had $A b$ titers that blocked SARS-CoV-2 RBD binding to ACE2 efficiently (BD80 > 10), only $18 \%$ and $11 \%$ of non-hospitalized symptomatic and asymptomatic individuals had Abs strongly interfering with ACE2 binding, respectively (Figure 1F). The proportion of non-hospitalized individuals with ACE2-blocking Abs correlated with the anti-RBD Ab binding titers, which parallel symptom severity (Figures 1D and 1G; Data S2). These results suggest that although all SARS-CoV-2-infected individuals can produce RBD-specific Abs, they may not be endowed with enough avidity or are not present at a sufficiently high concentration to block RBD binding to ACE2 effectively. This is illustrated by the large fraction of samples with RBD binding titers ranging between $10^{2}$ to $10^{3}$ and which did not block RBD binding to ACE2 efficiently (Figure $1 \mathrm{H}$ ). We found a positive correlation between the titers of Abs inhibiting RBD binding to ACE2 and neutralizing serum Ab titers (ID80) (Figure 1I), suggesting that the blockadeof-binding approach could be implemented as a highthroughput, alternative method to measuring serum nAb titers, as recently suggested (Tan et al., 2020).

Figure 1. Analysis of the Specificity of IgG, IgA, and IgM Serum/Plasma Abs from a Panel of 647 Hospitalized, Symptomatic, and Asymptomatic SARS-CoV-2-Infected Individuals

(A-C) Binding titers (ED50) of antigen-specific IgG (A), IgA (B), or IgM (C) were measured in plasma or sera from convalescent SARS-CoV-2 patients (47 hospitalized, 556 symptomatic, and 44 asymptomatic) and from pre-pandemic healthy donors $(n=32)$. A cut-off of 30 was determined based on signal of prepandemic samples and binding to uncoated ELISA plates.

(D) Binding titers (ED50) of S- and N-specific IgGs measured in sera from symptomatic and asymptomatic SARS-CoV-2-infected individuals from the Ticino healthcare workers cohort $(n=459)$ categorized according to symptoms severity, as described in the methods.

(E) IgG binding titers to SARS-CoV-2 RBD (left) and SARS-CoV-2 S pseudovirus neutralizing titers (ID80, center) before and after depletion of RBD-specific Abs from 21 SARS-CoV-2 immune plasma samples. The percentage of depletion of binding and neutralizing Abs (right) for each sample tested is shown on the right. (F) Ab-mediated inhibition of SARS-CoV-2 RBD binding to solid phase ACE2, as determined by ELISA. Shown is the reciprocal plasma or serum dilution that blocks $80 \%$ binding (BD80) of RBD to human ACE2.

(G) Ab-mediated inhibition of SARS-CoV-2 RBD binding to solid phase ACE2 in the Ticino healthcare workers cohort determined as in (F). A cut-off of 10 was used to separate neutralizing from non-neutralizing titers.

(H) Correlation analysis between levels of plasma/serum RBD-specific IgG (ED50) and the titers of Abs blocking RBD attachment to ACE2 (BD80).

(I) Correlation analysis between plasma/serum neutralizing Ab titers (ID80) and the titers of Abs blocking RBD attachment to ACE2 (BD80). 
A

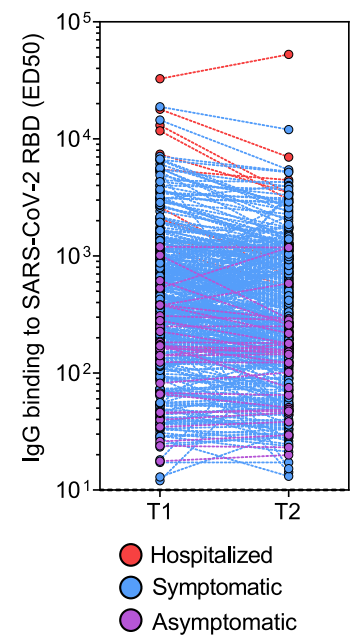

E

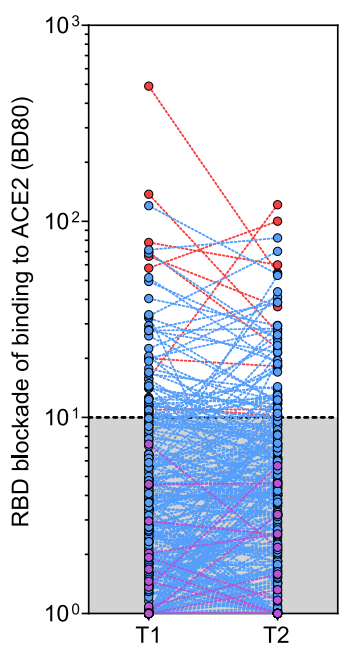

B

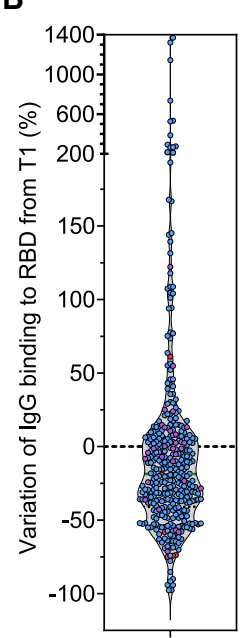

F

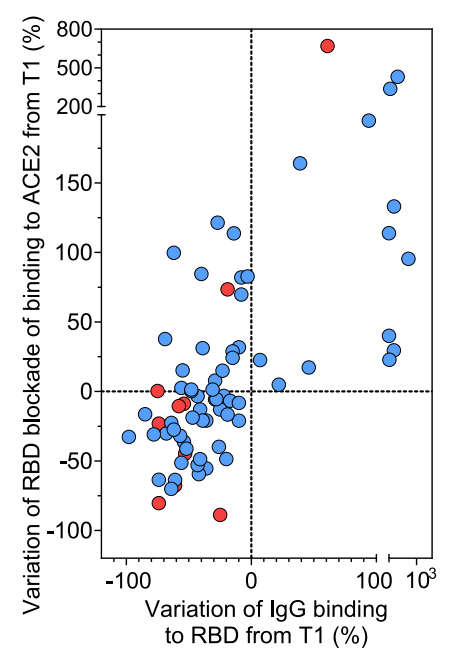

C
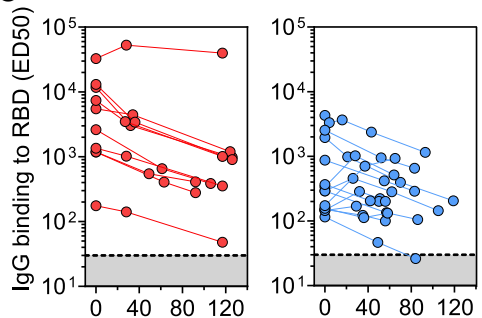

$\begin{array}{lllll}0 & 40 & 80 & 120\end{array}$

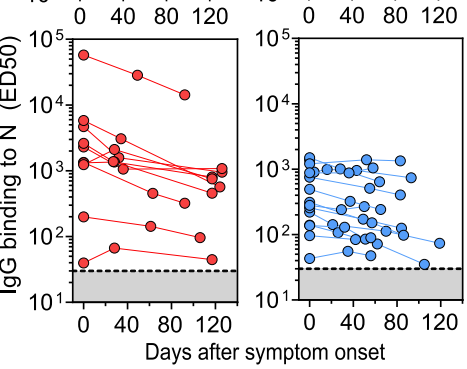

G
D
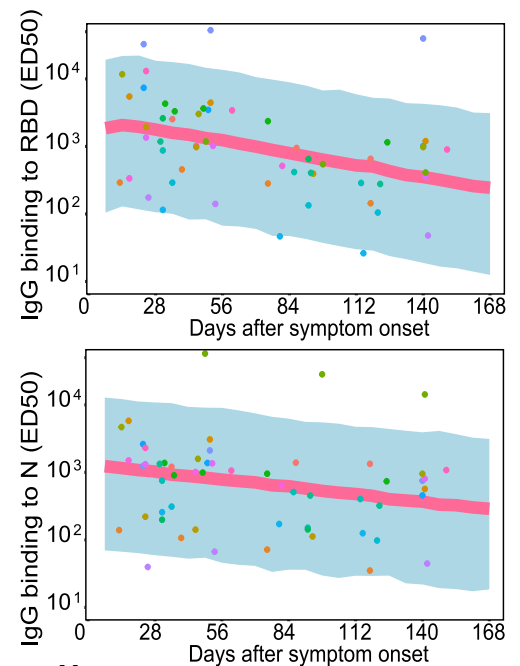

H

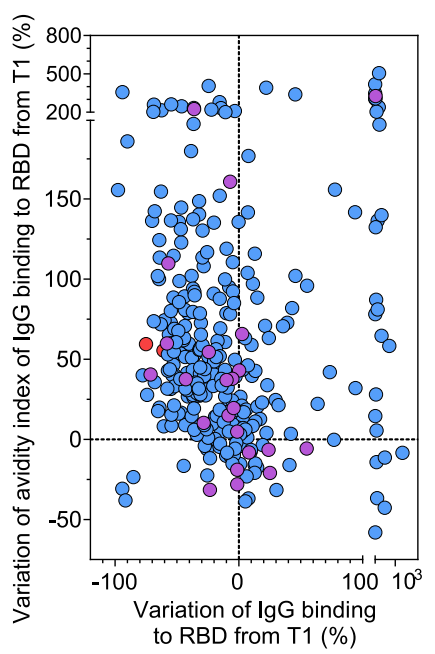

Figure 2. Kinetics of IgG Responses Specific for the SARS-CoV-2 RBD and Blocking RBD Attachment to ACE2

(A) Binding titers (ED50) of serum or plasma IgG to the SARS-CoV-2 RBD measured at two time points separated by an average time of 44 days in 368 subjects. $\mathrm{T} 1$, time of first blood draw; T2, time of second blood draw.

(B) Variation of RBD-specific IgG binding titers from T1 to T2.

(C) Kinetics of RBD- and N-specific IgG responses in serum or plasma from 24 convalescent individuals (red, hospitalized; blue, symptomatic non-hospitalized). The starting time point corresponds to the date of collection of the first sample.

(D) Model predicted longitudinal decline of RBD- and N-specific IgG binding titers from 18 convalescent individuals with respect to the onset of symptoms from infection. Symbols, observations; shaded region, 90\% prediction interval; line, median prediction.

(E) Serum or plasma titers of Abs blocking RBD attachment to ACE2 (BD80) measured at T1 and T2.

(F) Variation of RBD-specific IgG binding titers and titers of Abs blocking RBD attachment to ACE2 (BD80) from T1 to T2.

(G) Avidity index of serum IgG binding to RBD (\%) measured at T1 and T2.

(H) Variation of avidity index of IgG binding to RBD (\%) from T1 to T2.

Collectively, these findings indicate that the $A b$ responses to SARS-CoV-2 S are dominated by anti-RBD Abs and that the majority of the neutralizing activity against SARS-CoV-2 is mediated by RBD-specific Abs interfering with binding to ACE2.

\section{Kinetics of Ab Responses upon Natural SARS-CoV-2 Infection}

To characterize persistence of potentially protective Abs, we carried out a longitudinal analysis of IgG titers specific for
SARS-CoV-2 antigens at two time points (average of 44 days between samples) in 368 individuals tested within 3 months of infection. RBD-specific IgG titers declined by $35 \%$ on average between the two time points tested (Figures 2A and 2B), with a monthly average decay of approximately $25 \%$, and this trend was independent of the magnitude of the initial binding titers. We also followed the kinetics of RBD- and N-specific serum IgG over a period of up to 126 days from the collection of the first sample (approximately 150 days after onset of symptoms) for a 
A
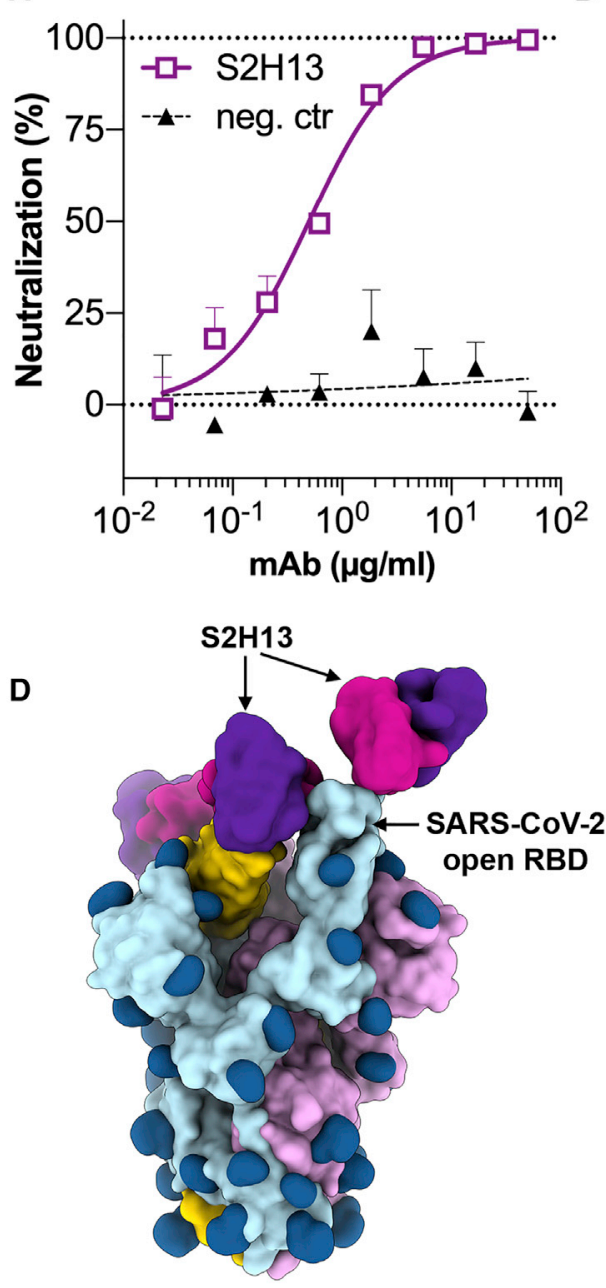

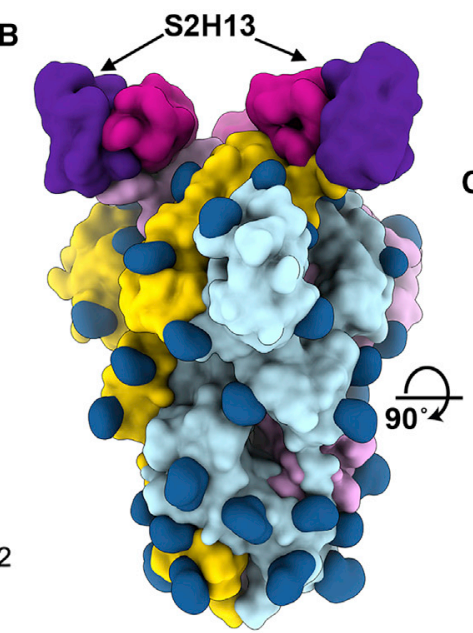

= Viral membrane

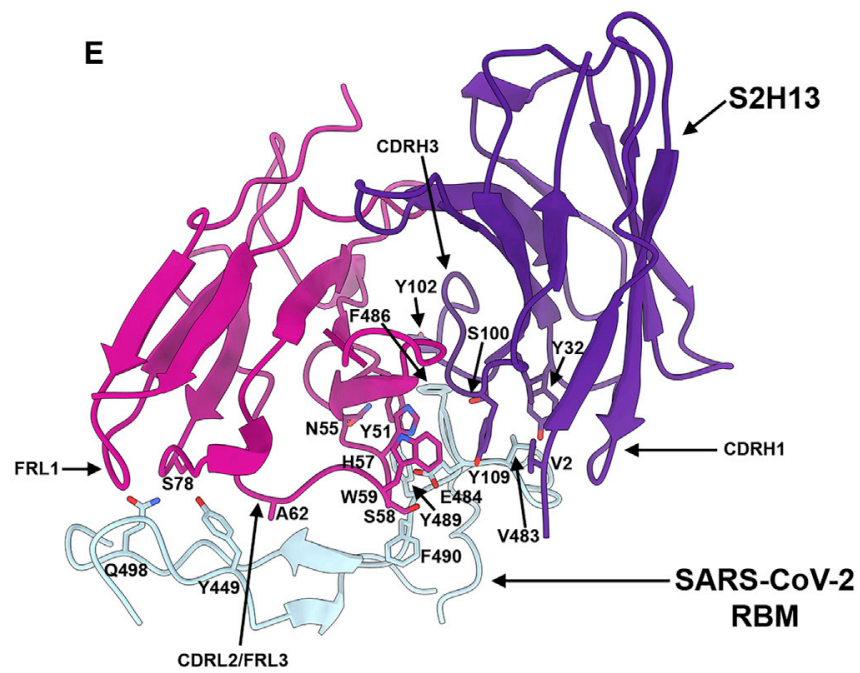

H Conserved

$\square$ SARS-CoV-2 S variants $\square$ SARS-CoV S variants
hACE2

loading

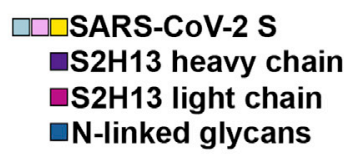

C

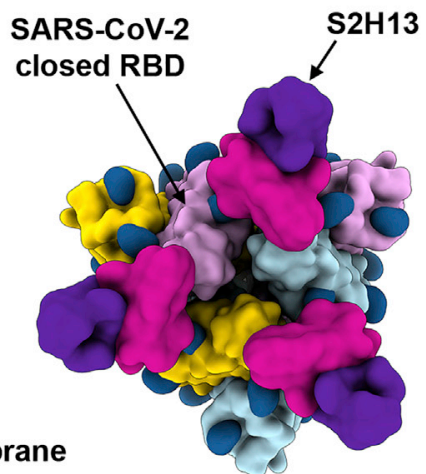

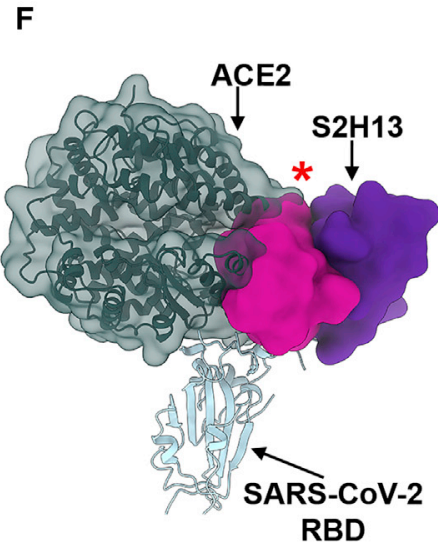

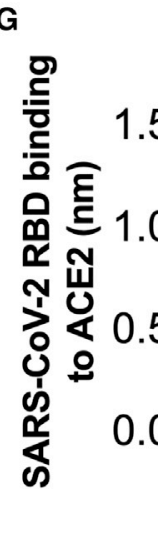

Figure 3. The S2H13 mAb Inhibits SARS-CoV-2 by Blocking Attachment to ACE2 via Recognition of an Epitope Accessible in the Open and Closed S Conformations

(A) SARS-CoV-2 S pseudovirus neutralization assay indicating an IC50 of $500 \mathrm{ng} / \mathrm{mL}$.

(B and C) Molecular surface representation of the SARS-CoV-2 S/S2H13 Fab complex structure with three RBDs closed shown in two orthogonal orientations. 
subset of 24 individuals (Figures 2C). This analysis revealed an average decay of RBD-specific IgGs of $67 \%$ over 4 months (21\% per month), consistent with the above finding with a larger cohort. To further characterize the decay kinetics of the RBDand $\mathrm{N}$-specific IgG following onset of disease symptoms, a longitudinal mixed effects model was employed in a subset of 18 convalescent, hospitalized and symptomatic, individuals who had available data on symptom onset from the start of infection (Figure 2D). The model predicted a half-life of 49 days for RBDspecific IgG Abs and 75 days for $\mathrm{S}$ - and $\mathrm{N}$-specific IgG Abs (Figure 2D), respectively. No significant differences were observed in the decay kinetics in the hospitalized compared to symptomatic individuals.

The kinetics of anti-RBD Ab titers blocking attachment to ACE2 did not mirror the overall decay observed for RBD-specific IgG. Indeed, we observed in the same samples an increase in Ab titers blocking attachment to ACE2 for $47 \%$ of the individuals who made this type of Abs (which account for $20 \%$ of subjects analyzed) (Figures 2E and 2F). This increase, which is not influenced by the initial titer of RBD-specific Abs, might result from the development of Abs with increasingly higher affinity, in the context of an overall waning of Abs titers targeting the RBD. Indeed, we measured an overall increased avidity of RBD-specific Abs between the two time points tested in support of this hypothesis (Figures $2 \mathrm{G}$ and $2 \mathrm{H}$ ).

Although we observed a progressive decay of Ab titers, our results demonstrate a change in the quality of the $A b$ responses that is expected to result in increased neutralizing activity based on the aforementioned correlation with blocking ACE2 attachment.

Structural Characterization of the S2H13 RBM-Specific Neutralizing mAb Recognizing Multiple RBD

\section{Conformers}

Given the heterogeneity of the humoral responses across individuals and the fact that the RBD is the prime target of neutralizing Abs, we set out to understand the fine specificity of RBD-targeting Abs elicited in SARS-CoV-2- or SARS-CoVexposed individuals (Figure S3A). To understand SARS-CoV-2 neutralization, we selected six mAbs with distinct function and epitope recognition (Figures S3A-S3C) from a large panel of RBD-specific Abs for structural characterization of their Fab fragments in complex with the SARS-CoV-2 S ectodomain trimer by cryoelectron microscopy (cryo-EM).

The $\mathrm{S} 2 \mathrm{H} 13$ neutralizing $\mathrm{mAb}$ was isolated from plasma cells of a SARS-CoV-2-infected individual 17 days after disease onset (Figures $3 A$ and S3A). In line with our previous work (Walls et al., 2020), cryo-EM characterization led to the identification of two conformational states corresponding to a closed $\mathrm{S}$ trimer and a trimer with one RBD open, each with three S2H13 Fabs bound, for which we determined $3 \mathrm{D}$ reconstructions at $3.0 \AA$ (with 3-fold symmetry) and $3.4 \AA$ (asymmetric) resolution, respectively (Figures $3 \mathrm{~B}-3 \mathrm{D}$ and S4A-S4E; Table S1). To improve the resolvability of the $\mathrm{S} 2 \mathrm{H} 13$ density, which was much lower than most other regions of the map, we used local refinement to determine a reconstruction at $\sim 3.5 \AA$ resolution enabling building the $\mathrm{S} 2 \mathrm{H} 13$ variable domains and its epitope (Figure S4F; Table S1).

$\mathrm{S} 2 \mathrm{H} 13$ recognizes an epitope located within the crevice formed by the receptor-binding motif (RBM) $\beta$-hairpin of the RBD, which is accessible in both the closed and open $S$ states, thereby explaining the stoichiometric binding of Fab to each protomer of the $S$ trimer (Figures 3B-3D, S5A, and S5B). S2H13 recognition of the SARS-CoV-2 RBM is mediated by electrostatic interactions and shape complementarity and is dominated by unusual contacts involving CDRL2/FRL3, accounting for $55 \%$ of the $\sim 700 \AA^{2}$ of surface area buried by the Fab, in addition to the 13-residue-long $\mathrm{CDRH} 3, \mathrm{CDRH} 1, \mathrm{FRL} 1$, and the heavy chain N-terminal end. Specifically, S2H13 FRL1 and CDRL2/FRL3 interact with the SARSCoV-2 residues 444-449, whereas the heavy chain $\mathrm{N}$ terminus, $\mathrm{CDRH} 1, \mathrm{CDRH} 3$, and CDRL2/FRL3 recognize the tip of the RBM spanning residues 472-498 (Figure 3E).

Superimposition of the SARS-CoV-2 RBD/ACE2 structures (Lan et al., 2020; Shang et al., 2020; Wang et al., 2020b; Yan et al., 2020) onto the SARS-CoV-2 S/S2H13 complex reveals that $\mathrm{S} 2 \mathrm{H} 13$ and ACE2 would clash upon binding to $S$ and that they share partially overlapping binding sites although they have almost orthogonal orientations relative to the RBD (Figure 3F). We confirmed these findings using biolayer interferometry and ELISA to show that $\mathrm{S} 2 \mathrm{H} 13$ competes with ACE2 for recognition of the SARS-CoV-2 RBD (Figures 3G and S3D). Therefore, $\mathrm{S} 2 \mathrm{H} 13$ likely neutralizes SARS-CoV-2 by preventing viral attachment to host cells via recognition of an $S$ epitope that remains accessible in both open and closed $S$ states, which is not the case for the ACE2-binding site.

Only 6 out of 20 residues within the $\mathrm{S} 2 \mathrm{H} 13$ epitope are conserved across SARS-CoV-2 and SARS-CoV, explaining the lack of binding to the latter virus (Figures 3H, S3B, and S6). Substitutions of 13 epitope residues have been reported among the $\sim 74,000$ SARS-CoV-2 isolates sequenced to date, indicating that potential escape mutants might have already emerged to this site (Figures $3 \mathrm{H}$ and S3F; Table S2).

\section{Structural Characterization of the Neutralizing S2H14 mAb Targeting an RBM Epitope Accessible Uniquely in the Open S State}

The $\mathrm{S} 2 \mathrm{H} 14$ neutralizing mAb was isolated from the plasma cells from the same SARS-CoV-2 convalescent individual from which

(D) Molecular surface representation of the SARS-CoV-2 S/S2H13 Fab complex structure with one RBD open. Each SARS-CoV-2 protomer is colored distinctly (cyan, pink, and gold), and $\mathrm{N}$-linked glycans are rendered as dark blue surfaces. The $\mathrm{S} 2 \mathrm{H} 13$ light and heavy chain variable domains are colored magenta and purple, respectively.

(E) $\mathrm{S} 2 \mathrm{H} 13$ recognizes a crevice formed by the SARS-CoV-2 RBM. Selected side chains at the interface are shown.

(F) S2H13 and ACE2 (dark green) bind overlapping RBM epitope. The red star indicates steric clashes.

(G) BLI binding competition between S2H13 and ACE2 for binding to the SARS-CoV-2 RBD.

(H) Molecular surface representation of the SARS-CoV-2 RBD (gray) with the S2H13 epitope colored by residue conservation across SARS-CoV-2 isolates and SARS-CoV. 
A

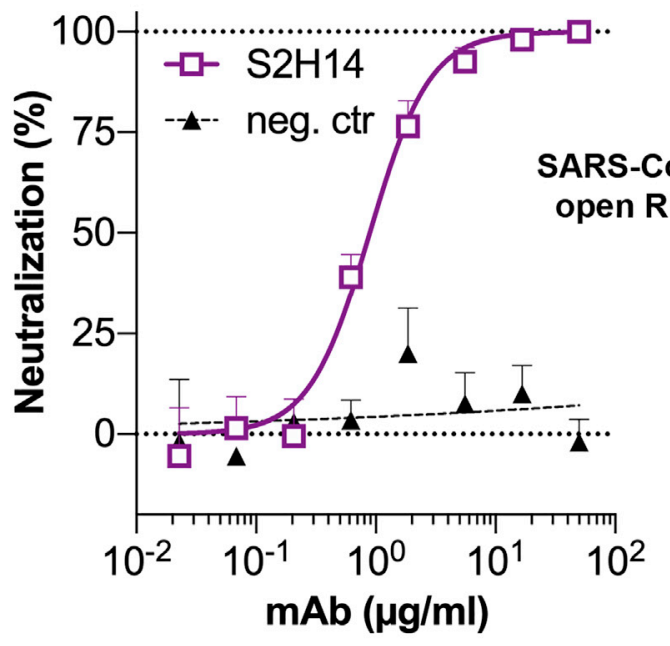

B
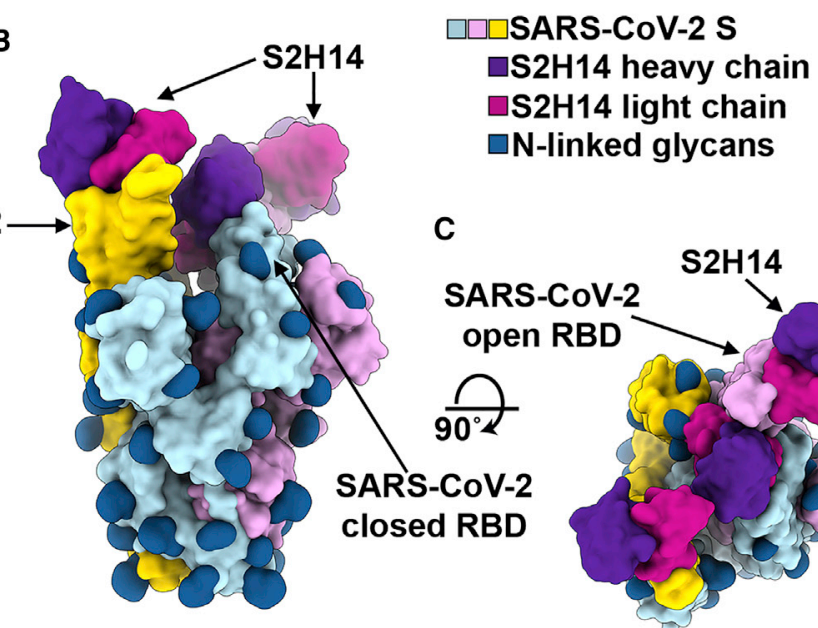

$=$ Viral membrane

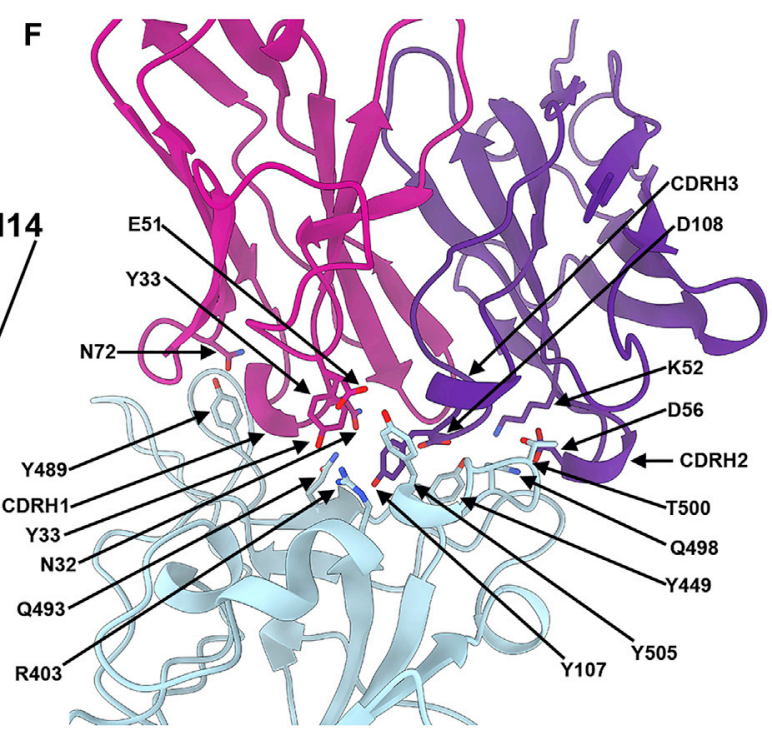

conserved

I $\square$ SARS-CoV-2 S variants $\square$ SARS-CoV S variants
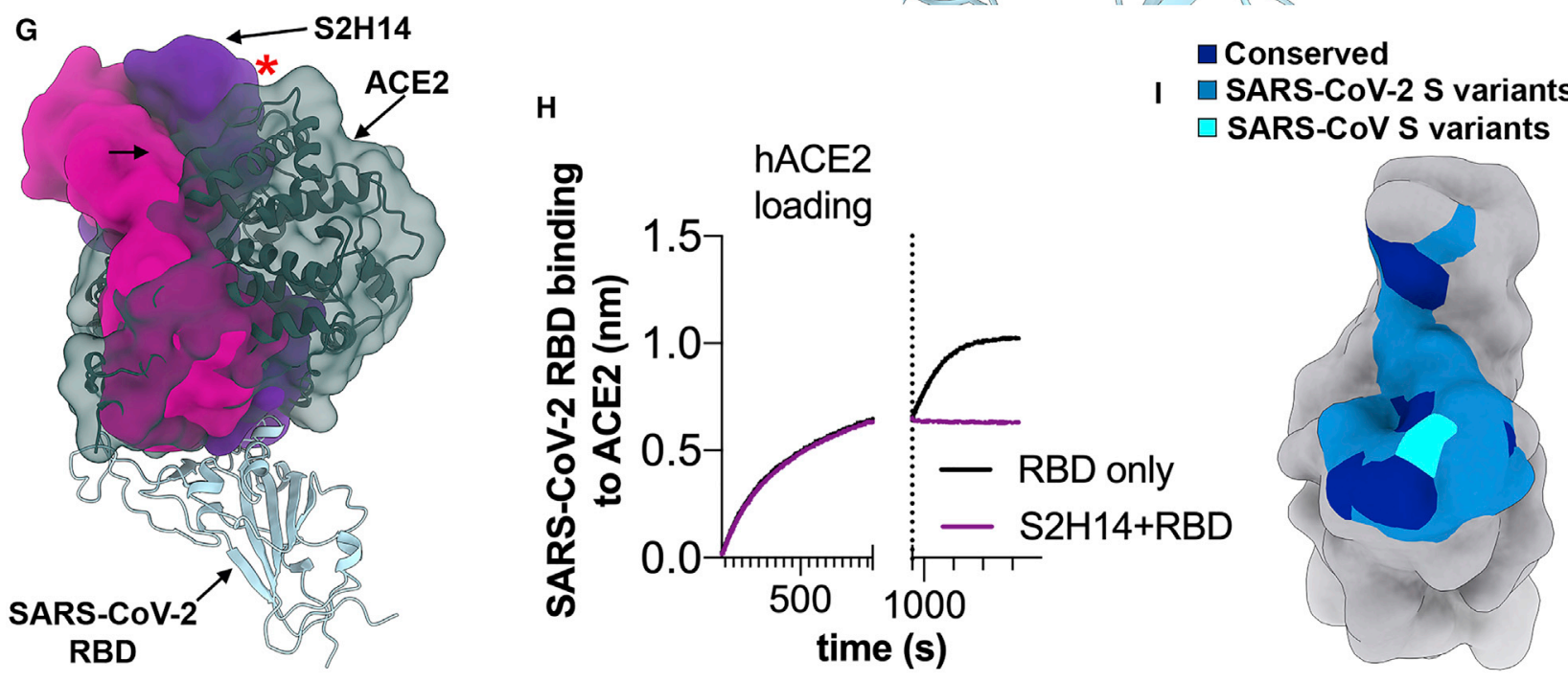
$\mathrm{S} 2 \mathrm{H} 13$ was obtained and does not carry somatic hypermutations in the heavy or light chain variable regions (Figures $4 \mathrm{~A}$ and S3A).

Cryo-EM analysis showed that a subset of the selected particle images corresponded to an S trimer with one RBD closed and two RBDs open (Figures $4 \mathrm{~B}$ and $4 \mathrm{C}$ ), whereas the rest of the data featured an $S$ trimer with all three RBDs open (Figures $4 \mathrm{D}$ and $4 \mathrm{E}$ ). In agreement with binding data, S2H14 recognizes the RBD (Figures S3B and S5A) and each of the three RBDs interacted with an $\mathrm{S} 2 \mathrm{H} 14 \mathrm{Fab}$ in both conformational states. We determined asymmetric 3D reconstructions for each of the two conformational states at $7.8 \AA$ and $8.5 \AA$ resolution, respectively, along with crystal structures of the $\mathrm{S} 2 \mathrm{H} 14 \mathrm{Fab}$ and of the RBD bound to the $\mathrm{S} 2 \mathrm{H} 14, \mathrm{~S} 309$ and S304 Fabs at $2.5 \AA$ and $2.65 \AA$ resolution, respectively (Figures $4 \mathrm{~B}-4 \mathrm{E}$ and $\mathrm{S} 4 \mathrm{G}-\mathrm{S} 4 \mathrm{~K}$; Tables S1 and S3).

The observation of SARS-CoV-2 S trimers with two and three RBDs open suggests that $\mathrm{S} 2 \mathrm{H} 14$ binding conformationally selects open RBDs in a way reminiscent of the SARS-CoV S230 (Walls et al., 2019) and of the SARS-CoV-2 C105 (Barnes et al., 2020) neutralizing mAbs. Indeed, these findings differ from what we observed for SARS-CoV-2 S without (Walls et al., 2020) or with bound Fabs, such as S309 (Pinto et al., 2020) or $\mathrm{S} 2 \mathrm{H} 13$, which recognize epitopes accessible in all prefusion $\mathrm{S}$ states, hence no conformational selection occurred. Although the $\mathrm{S} 2 \mathrm{H} 14$ epitope is masked in the closed S trimer, our cryoEM data show that opening of two RBDs is enough to allow three Fabs to bind to an $\mathrm{S}$ trimer, as the remaining closed RBD can engage a Fab due to its angle of approach (Figures 4B and 4C).

$\mathrm{S} 2 \mathrm{H} 14$ recognizes an epitope overlapping with the RBM, which is inaccessible in the closed $S$ state but becomes exposed upon RBD opening (Figures 4B-4E), similar to the ACE2-binding site (Walls et al., 2020; Wrapp et al., 2020). The crystal structure of the RBD bound to the S2H14, S309, and S304 Fabs show that CDRH1-H3 and CDRL1-L3 participate in the CDRH3-dominated $\mathrm{S} 2 \mathrm{H} 14$ paratope which buries $900 \AA^{2}$ at the interface with the RBM. The epitope spans the entire RBM crevice and involves SARS-CoV-2 S residues 403, 444-456, 475, and 485-505 that interact with $\mathrm{S} 2 \mathrm{H} 14$ via hydrogen-bonding and shape complementarity (Figure S6A).

The structures show that $\mathrm{S} 2 \mathrm{H} 14$ and ACE2 bind to largely overlapping sites in the RBM (Figure 4F) and would be sterically incompatible with simultaneous binding to a single SARS-CoV-2 RBD (Figure 4G). We validated this observation using biolayer interferometry and ELISA to show that S2H14 competed with ACE2 for recognition of the SARS-CoV-2 RBD (Figures $4 \mathrm{H}$ and
S3D), indicating that S2H14 likely neutralizes SARS-CoV-2 through inhibition of virus/host cell interaction.

We found that 13 out of 23 epitopes residues are substituted between SARS-CoV-2 and SARS-CoV, thereby rationalizing the lack of cross-reactivity of $\mathrm{S} 2 \mathrm{H} 14$ with the latter virus (Figures 4I, S3B, and S6; Table S2). Moreover, SARS-CoV-2 variants have already been detected for 15 epitope residues, which suggests that some of the viruses currently circulating in humans might be able to escape $\mathrm{S} 2 \mathrm{H} 14$-mediated neutralization (Figure S3F).

\section{The S2A4 mAb Recognizes a Cryptic Epitope Leading to} Release of the $S_{1}$ Subunit

The S2A4 mAb was isolated from memory B cells of a hospitalized patient 24 days after disease onset and was found to weakly neutralize SARS-CoV-2 infection (Figures 5A and S3A).

$2 \mathrm{D}$ and $3 \mathrm{D}$ classification of the cryo-EM dataset revealed the presence of three distinct open conformations of the $S$ trimer, with three bound S2A4 Fabs and RBDs swung out to various extent, as well as an $\mathrm{S}_{1}$ subunit trimer class (Figures $5 \mathrm{~B}-5 \mathrm{C}$ and $S 7 A-S 7 H)$. We determined $3 D$ reconstructions of the three open conformations of the S/S2A 4 complex at $3.3 \AA$ resolution (applying 3-fold symmetry) and at $3.8 \AA$ and $3.9 \AA$ resolution (asymmetric) (Figures 5B and 5C and S7A-S7F; Table S1). To improve the resolution of the S2A4 density, which was lower than the overall map resolution, we used local refinement to yield a reconstruction at $3.6 \AA$ resolution allowing to build the S2A4 variable domains and the epitope, which we subsequently validated by determining a crystal structure of the S2A4 Fab at $2.5 \AA$ resolution (Figures S7D and S7E; Tables S1 and S3). Furthermore, we obtained a low-resolution reconstruction of the $\mathrm{S}_{1}$ subunit trimer bound to three S2A4 Fabs (Figures S7G and $\mathrm{S} 7 \mathrm{H})$.

S2A4 binds to a cryptic epitope (distinct from the RBM) requiring opening of two adjacent $R B D$ s to be unmasked and allow Fab binding (Figures $5 \mathrm{~B}$ and $5 \mathrm{C}$ ). This finding along with the detection of an $S_{1}$ subunit trimer class, which we interpret as being a triggered $S$ with a disordered fusion machinery remaining covalently linked (Figure S3G), suggest that S2A4 acts as a molecular ratchet biasing the SARS-CoV-2 S conformational equilibrium toward opened RBDs. We confirmed these results by showing that $\mathrm{S} 2 \mathrm{~A} 4$ promoted shedding of the $\mathrm{S}_{1}$ subunit from cell-surface-expressed full-length wild-type $S$, as was the case with the RBM-targeted S2H14 (Figure S3E). Our data are also in line with previous reports of the SARS-CoV neutralizing mAb S230- and ACE2-mediated transition of SARS-CoV S

Figure 4. The S2H14 mAb Inhibits SARS-CoV-2 by Blocking Attachment to the ACE2 Receptor

(A) SARS-CoV-2 S pseudovirus neutralization assay indicating an IC50 of $900 \mathrm{ng} / \mathrm{mL}$.

(B and C) Molecular surface representation of the SARS-CoV-2 S/S2H14 Fab complex structure with two RBDs open and one RBD closed viewed along two orthogonal orientations.

(D and E) Molecular surface representation of the SARS-CoV-2 S/S2H14 Fab complex structure with three RBDs open shown in two orthogonal orientations. Each SARS-CoV-2 protomer is colored distinctly (cyan, pink, and gold), and N-linked glycans are rendered as dark blue surfaces. The S2H14 light and heavy chain variable domains are colored magenta and purple, respectively.

(F) S2H14 binds to an epitope within the SARS-CoV-2 RBM.

(G) S2H14 and ACE2 (dark green) bind overlapping RBM epitope. The red star indicates steric clashes. (H) BLI binding competition between S2H14 and ACE2 for binding to the SARS-CoV-2 RBD.

(I) Molecular surface representation of the SARS-CoV-2 RBD (gray) with the S2H14 epitope colored by residue conservation across SARS-CoV-2 isolates and SARS-CoV. 
from the prefusion to the postfusion states (Song et al., 2018; Walls et al., 2019), the cryo-EM observation of $S_{1}$ subunit trimers released from the MERS-CoV $S$ ectodomain upon cleavage at $S_{1} / S_{2}$ (Yuan et al., 2017) and the fact that $S$ spontaneously refolds to the postfusion state in the absence of the $S_{1}$ subunit (Walls et al., 2017).

S2A4 binding to the RBD buries an average surface of $\sim 850 \AA^{2}$ using all six CDR loops along with contributions from $\mathrm{FRH} 3$ and FRL3. CDRH3 and CDRL1 dominate the interface, which involves electrostatic and hydrophobic interactions (Figures 5D and $5 E$ ). The S2A4 epitope comprises residues 368-388, which form two $\alpha$ helices and an intervening $\beta$ strand participating in the formation of the structurally conserved RBD $\beta$ sheet, and residues $407-414$ forming an $\alpha$ helix followed by a loop segment (Figures 5D, 5E, and S6A).

S2A4 recognizes an epitope distinct from the RBM and its footprint does not overlap with the ACE2-binding site (Figure 5F). However, our cryo-EM structure indicates that upon binding, S2A4 would sterically clash with ACE2 interacting with the same protomer within an $S$ trimer. We used biolayer interferometry and ELISA to validate these structural findings and demonstrated that S2A4 and ACE2 compete for binding to the SARS-CoV-2 RBD indicating that the neutralizing activity of this $A b$ likely results from preventing viral attachment to its host cell receptor (Figures 5G and S3D).

Sixteen out of 19 epitope residues are conserved across SARS-CoV-2 and SARS-CoV $S$ glycoproteins (Figures $5 \mathrm{H}$, S3F, and S6A; Table S2). However, S2A4 does not cross-react with the SARS-CoV RBD putatively due to steric hindrance with a glycan at position N357, which is absent in the SARSCoV-2 RBD (Figures 5H, S3B, and S6A).

Identification of a SARS-CoV-2 S Cryptic Supersite Defined by the Cross-Reactive $\$ 304$ mAb along with S2A4, S2X35, and CR3022 mAbs

We previously isolated from a SARS survivor two weakly neutralizing, cross-reactive mAbs (S304 and S315) that bind the RBD at sites distinct from both the RBM and the S309 epitope (Figures S3A-S3C) (Pinto et al., 2020). Cocktails containing either of these two mAbs with S309 led to synergistic enhancement of the S309 neutralization potency against SARS-CoV-2 (Pinto et al., 2020).

Similar to S2A4, 3D classification of the cryo-EM data for the S/S304 complex revealed the presence of three distinct open conformations of the $S$ trimer, with three bound Fabs and RBDs swung out to various extents, as well as an $S_{1}$ subunit trimer class bound to three S304 Fabs (Figures 6A-6C and S7I-S7O; Table S1). We determined a 3D reconstruction at $4.3 \AA$ resolution (applying 3-fold symmetry) for one of the open $S$ states and at $8 \AA$ resolution (asymmetric) for the other two classes (Figures 6A, 6B, and S7I-S7M; Table S1). Furthermore, we obtained a $10 \AA$ resolution cryo-EM reconstruction of the $S_{1}$ subunit trimer with three bound S304 Fabs (Figures 6C, S7N, and $\mathrm{S} 7 \mathrm{O})$.

S304 recognizes a cryptic epitope, which is buried in the closed $\mathrm{S}$ conformation but is distinct from the RBM, with one S304 Fab bound to each of the three open RBDs (Figures 6A and 6B). CDRH1-H3, CDRL1, and CDRL3 interact with SARSCoV-2 S through burial of an average surface area of $900 \AA^{2}$ at the epitope/paratope interface involving electrostatic interactions and shape complementarity (Figure 6D). Based on the crystal structure of the RBD/S304/S309/S2H14 complex, the S304 epitope comprises residues 369-392, which are part of two $\alpha$ helices and an intervening $\beta$ strand, as well as residues $515-517$ (both regions participating in the formation of the structurally conserved RBD $\beta$ sheet) and loop residues 411-414 and 427430 (Figures 6D and S6A; Table S3). Although S304 binds away from the RBM, we observed partial competition between S304 and ACE2 for binding to the SARS-CoV-2 RBD, which might be explained by steric hindrance with the ACE2 N322 glycan and/or with the ACE2 N terminus (through the heavy chain constant domain of S304 bound to a neighboring protomer) (Figures $6 \mathrm{E}$ and S3D).

Cross-reactivity of S304 with SARS-CoV-2 S and SARS-CoV $S$ is explained by the conservation of 23 out of 25 epitope residues with neither of the two substitutions (PSARS-CoV-2 $384 A_{\text {SARS-Cov }}$ and $T_{\text {SARS-CoV-2 }} 430 M_{\text {SARS-Cov) predicted to affect }}$ binding in light of our structural data (Figures 6F, S3B, and $\mathrm{S} 6 \mathrm{~A})$. The conserved nature of the $\mathrm{S} 304$ epitope among sarbecoviruses indicate that S304 is likely to cross-react with other related sarbecoviruses (Figures S3F and S6B; Table S2).

The S304 epitope partially overlaps with the epitopes of the weakly neutralizing mAb CR3022 (Huo et al., 2020; Joyce et al., 2020; ter Meulen et al., 2006; Tian et al., 2020; Yuan et al., 2020) and of the neutralizing mAb S2A4 (Figure 6G). It also overlaps with the mAb S2X35, which we isolated from the memory B cells of a COVID-19 convalescent symptomatic individual 48 days after disease onset and which neutralizes entry of SARS-CoV-2 pseudovirus into cells with an IC50 of $500 \mathrm{ng} / \mathrm{mL}$ (Figures $6 \mathrm{H}$ and S3A-S3C; Data S3). Although these mAbs have distinct angles of approach (Figures 6E, 6G, and 6H), they conformationally select for open RBDs through recognition of cryptic epitopes requiring opening of at least two RBDs for binding and lead to release of the $S_{1}$ subunit (Figure S3E). Comparison of the binding poses of these mAbs relative to the SARSCoV-2 RBD reveals that their neutralization potencies correlate with the Fab proximity to the RBM. Both S2X35 and S2A4 Fabs sterically clash with ACE2 and are more potent neutralizers than S304, which putatively only partially overlap with ACE2 (Figures $6 \mathrm{E}, 6 \mathrm{G}, 6 \mathrm{H}$, and S3D). Collectively, these data suggest that the ability to hinder ACE2 binding by some mAbs recognizing this cryptic supersite largely explains their neutralization potencies.

\section{Fc-Mediated Effector Activation Mechanisms by RBD-} Specific Neutralizing mAbs

Natural killer-dependent mAb-mediated cell cytotoxicity (ADCC) or macrophage/dendritic cell-dependent mAb-mediated cellular phagocytosis (ADCP) can participate in controlling infections by clearing viruses and infected cells and by stimulating $T$ cell response via presentation of viral antigens (DiLillo and Ravetch, 2015; He et al., 2017). Among the six mAbs used in this study, only $\mathrm{S} 2 \mathrm{H} 13$ and S309 promoted ADCC as measured by Fc $\gamma$ RIIla (V158 allele) activation (Figure S3H). A weak activation of Fc $\gamma R$ Illa, which is a reporter for ADCP, was observed for $\mathrm{S} 2 \mathrm{H} 13, \mathrm{~S} 2 \mathrm{H} 14$, and $\mathrm{S} 2 \mathrm{X} 35$, as compared to the robust activation previously observed with mAb S309 (Pinto et al., 2020) (Figure S3I). Similar to what we observed for ADCC, only S2H13 
A

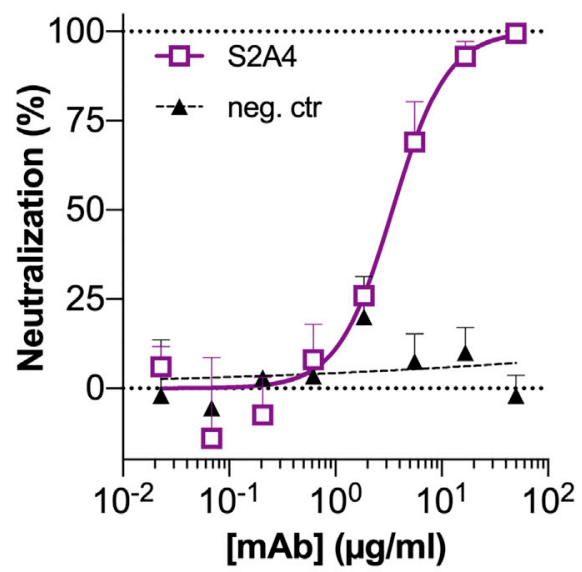

B

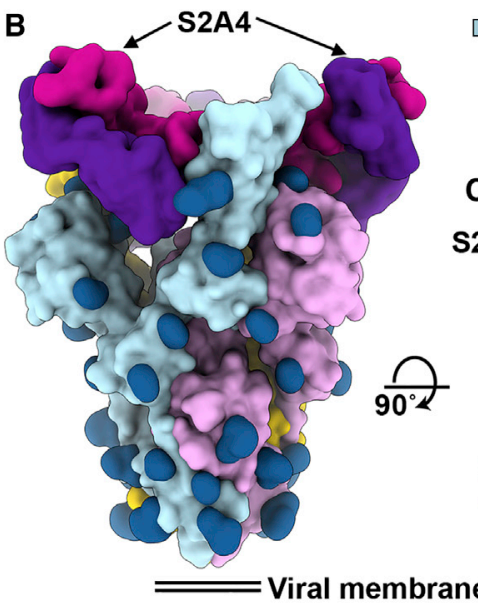

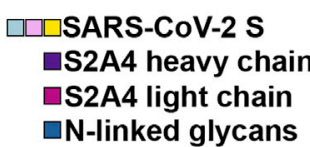

C

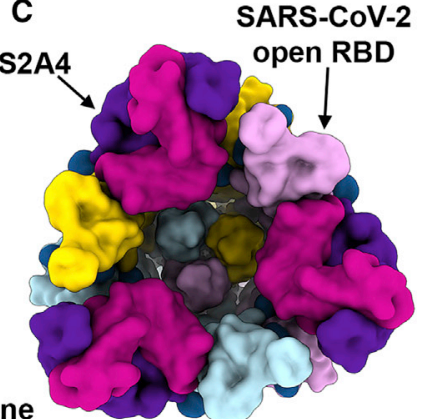

D

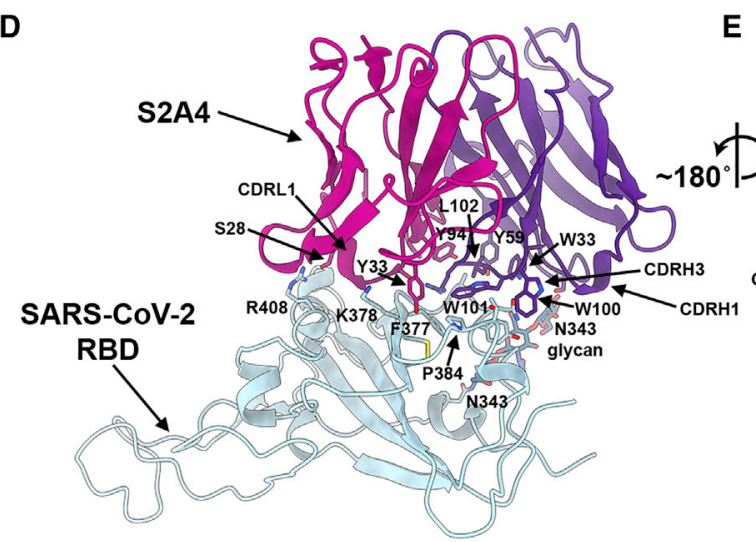

$\mathbf{F}$

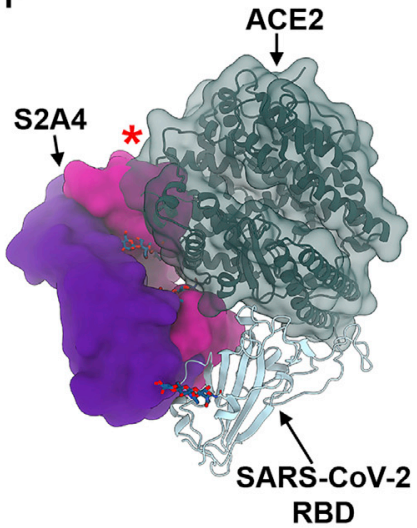

G

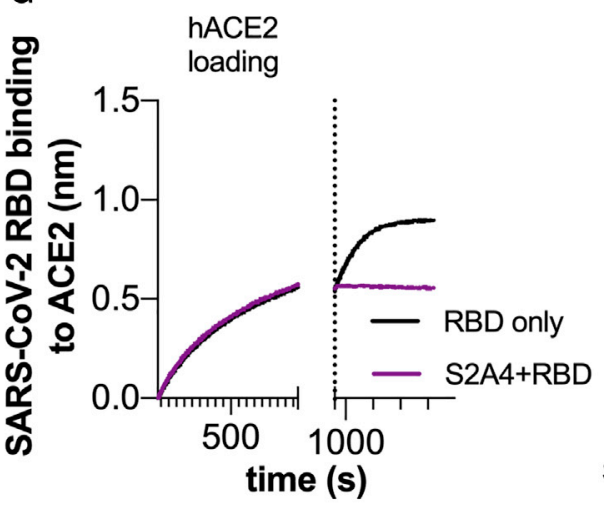

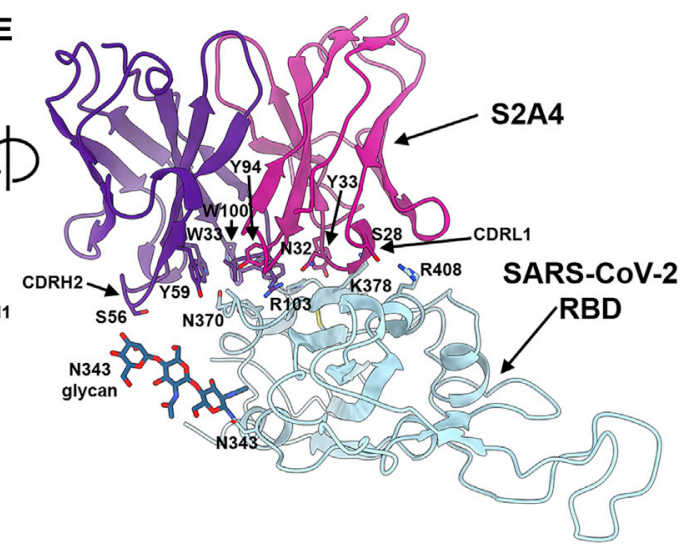

H

$$
\begin{aligned}
& \text { Conserved } \\
& \square \text { SARS-CoV S variants }
\end{aligned}
$$

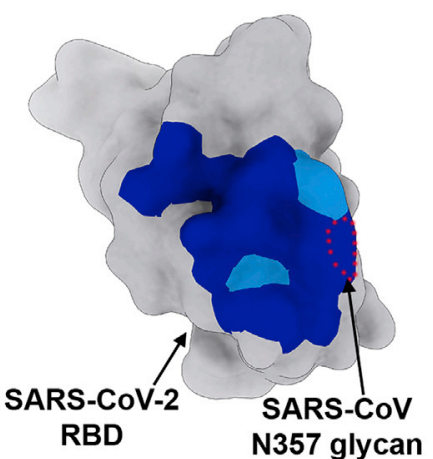

Figure 5. The S2A4 mAb Promotes SARS-CoV-2 S Opening through Binding to a Cryptic Epitope (A) SARS-CoV-2 S pseudovirus neutralization assay indicating an IC50 of $3.5 \mu \mathrm{g} / \mathrm{mL}$.

(B and C) Molecular surface representation of the SARS-CoV-2 S/S2A4 Fab complex cryo-EM structure with three RBDs open viewed along two orthogonal orientations. Each SARS-CoV-2 protomer is colored distinctly (cyan, pink, and gold), and N-linked glycans are rendered as dark blue surfaces. The S2A4 light and heavy chains are colored magenta and purple, respectively.

(D and E) Zoomed-in views of the contacts formed between S2A4 and the RBD with selected side chains shown.

(F) S2A4 and ACE2 (dark green) bind distinct RBD epitopes but would clash via steric hindrance. The red star indicates steric clashes.

(G) BLI binding competition between S2A4 and ACE2 for binding to the SARS-CoV-2 RBD.

(H) Molecular surface representation of the SARS-CoV-2 RBD (gray) with the S2A4 epitope colored by amino acid residue conservation with SARS-CoV. The position of the SARS-CoV N357 glycan is indicated with red dotted lines. 
A

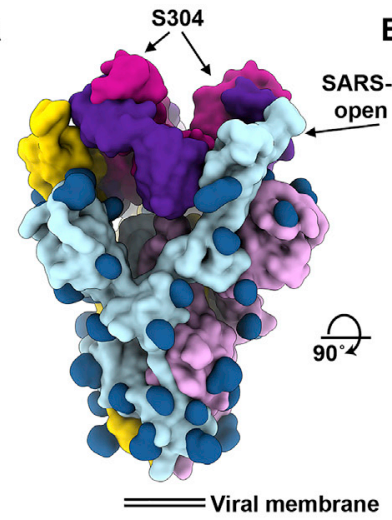

$B$

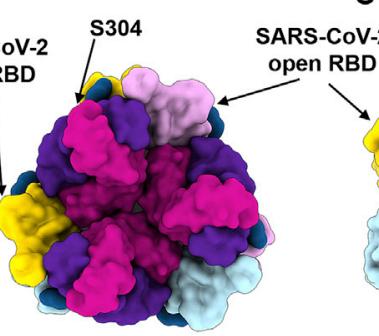

口U⿴囗十 SARS-CoV-2 S $\square$ S304 heavy chain

$\square 304$ light chain $\square$ N-linked glycans

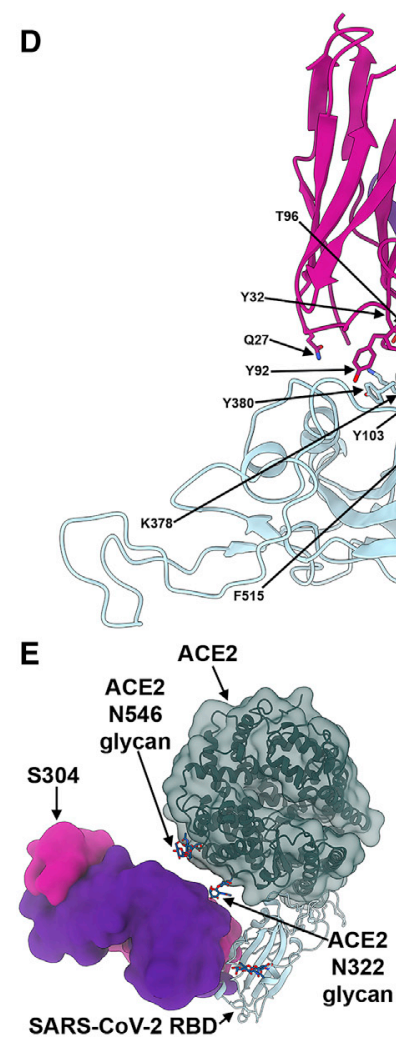

C

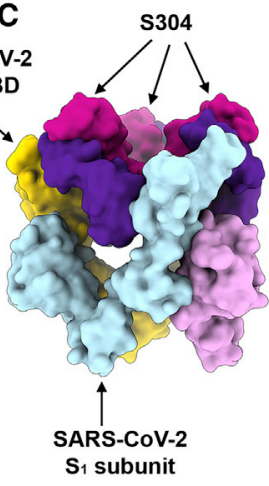

$\mathbf{F}$

- Conserved

a SARS-CoV-2 S variants

Figure 6. The S304 mAb Promotes SARSCoV-2 S Opening through Binding to a Cryptic Epitope Conserved within the Sarbecovirus Subgenus

( $A$ and $B$ ) Molecular surface representation of the SARS-CoV-2 S/S304 Fab complex cryo-EM structure with three RBDs opened viewed along two orthogonal orientations. Each SARS-CoV-2 S protomer is colored distinctly (cyan, pink, and gold), and $\mathrm{N}$-linked glycans are rendered as dark blue surfaces. The S304 light and heavy chains are colored magenta and purple, respectively.

(C) Cryo-EM reconstruction of the $\mathrm{S}_{1}$ subunit trimer (with disordered $\mathrm{S}_{2}$ ) bound to three S304 Fabs viewed along two orthogonal orientations and the corresponding atomic model fit in density. Each SARS-CoV-2 $\mathrm{S}_{1}$ protomer is colored distinctly (cyan, pink, and gold). The S304 light and heavy chains are colored magenta and purple, respectively.

(D) Ribbon diagram of the crystal structure of $\mathrm{S} 304$ (pink and purple), $\mathrm{S} 2 \mathrm{H} 14$, and $\mathrm{S} 309$ in complex with the SARS-CoV-2 RBD (light blue). Only the $\mathrm{S} 304$ variable domains are shown, whereas $\mathrm{S} 2 \mathrm{H} 14$ and S309 were omitted for clarity.

(E) Positioning of ACE2 (dark green) relative to the S304 Fab bound to the SARS-CoV-2 RBD. ACE2 $\mathrm{N}$-linked glycans at position N322 and N546 are indicated, as they could putatively clash with S304. (F) Molecular surface representation of the SARSCoV-2 RBD (gray) with the S304 epitope colored by residue conservation with SARS-CoV.

( $G$ and $H$ ) Positioning of ACE2 (dark green) relative to the S2A4 (G) and S2X35 (H) Fabs bound to the SARS-CoV-2 RBD. The red stars indicate steric clashes.

RBD antigenic map (Figures $7 \mathrm{~A}$ and $\mathrm{S} 3 \mathrm{C})$. $\mathrm{S} 2 \mathrm{H} 14$ and $\mathrm{S} 2 \mathrm{H} 13$ define two classes of RBM-targeting mAbs recognizing sites that we define here as la and $\mathrm{Ib}$, respectively. Site la largely overlaps with the ACE2-binding site and is only accessible in the open $S$ state (Figure $7 \mathrm{~B}$ ), whereas site Ib partially overlaps with the ACE2 footprint and is accessible in both

and S309 promoted complement-dependent cytotoxicity (Figure S3J). These findings may result from the different orientation and/or positioning of the S-bound $\mathrm{mAb} F \mathrm{~F}$ fragments relative to Fc $\gamma R$ IIlla and Fc $\gamma$ Rlla receptors, as well as to the C1q subcomponent of the classical complement pathway, and suggests that only a fraction of RBD-specific Abs can recruit Fc-dependent protective mechanisms in vivo, as previously shown for other antiviral Abs (Corti et al., 2011; Hessell et al., 2007; Pinto et al., 2020).

\section{Definition of Humoral Immunodominant Responses in SARS-CoV-2-Infected Individuals}

The epitopes recognized by the 5 aforementioned structurally characterized human mAbs along with $\mathrm{S} 309$ cover a large fraction of the SARS-CoV-2 RBD surface and collectively define an the open and closed $S$ states (Figure $7 C$ ). These epitopes are SARS-CoV-2-specific and harbor several naturally occurring mutations among circulating viral isolates (Figure S3F; Table S2). The S2X35, S2A4, and S304 mAbs recognize overlapping cryptic epitopes that are only accessible when at least two RBDs are open, respectively termed sites Ila, Illb, and Ilc, which are positioned increasingly further away from the ACE2-binding site (Figures 7D-7F). Finally, S309 binds to a conserved epitope termed site IV, which is accessible independently of the RBD conformation, and neutralizes SARS-CoV-2 without interfering with ACE2 binding (Figure 7G) (Pinto et al., 2020).

S309 and S2H13 are set apart from the other mAbs studied here as they recognize epitopes accessible in both the closed and open $S$ states. Consistent with a recent report that the closed S state is favored at endosomal pH (Zhou et al., 2020b), 
A

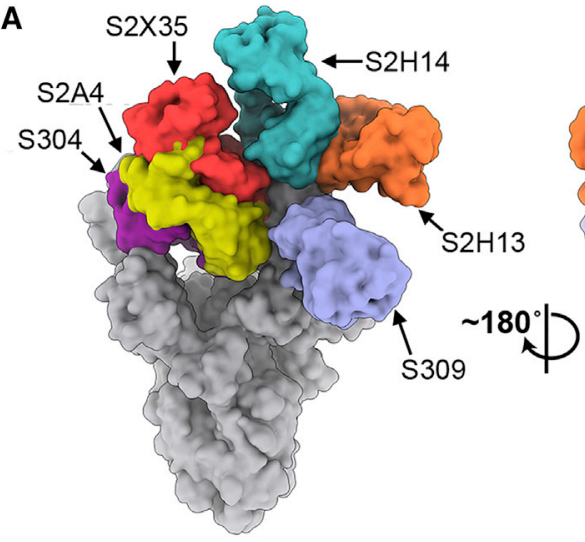

B

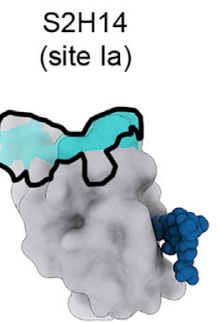

E

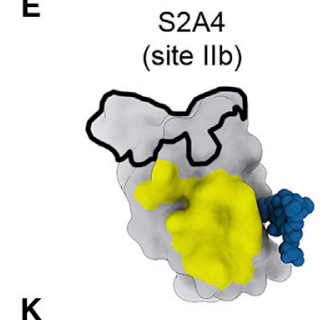

K
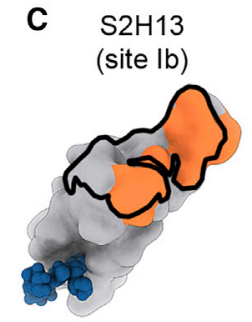

F $\mathrm{S} 304$ (site Ilc)
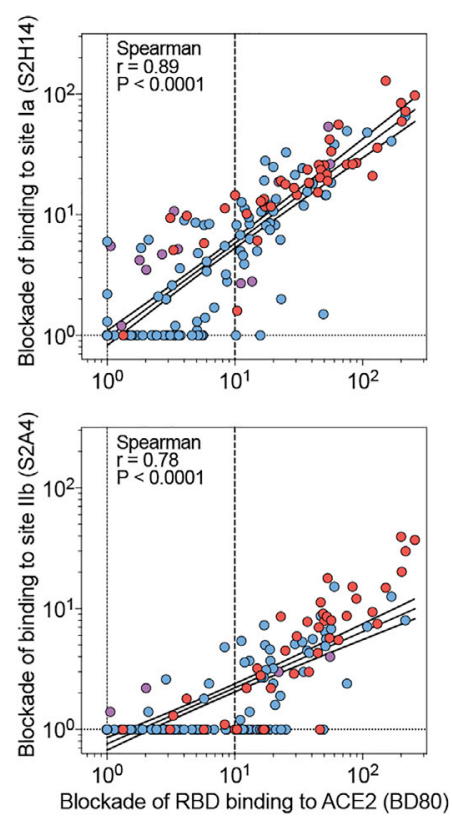
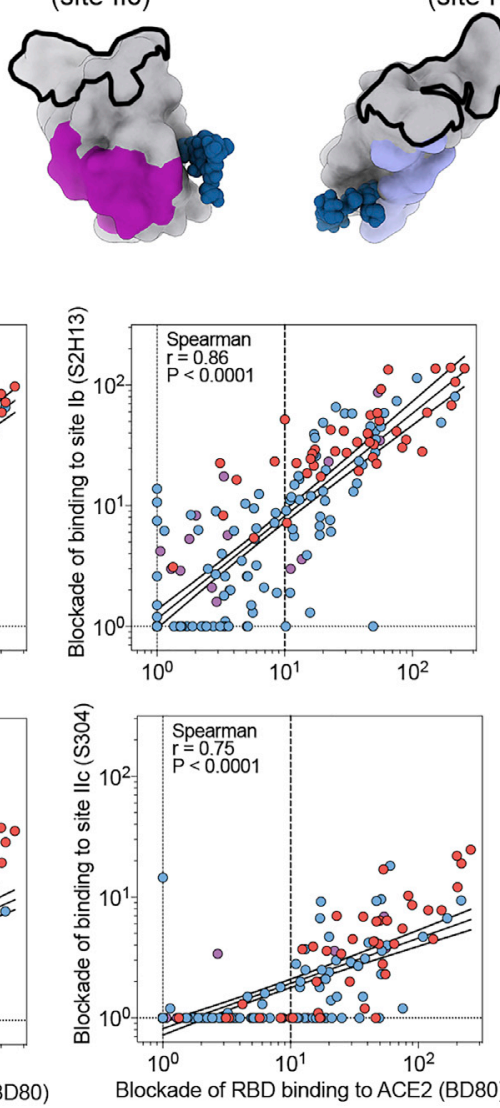

H
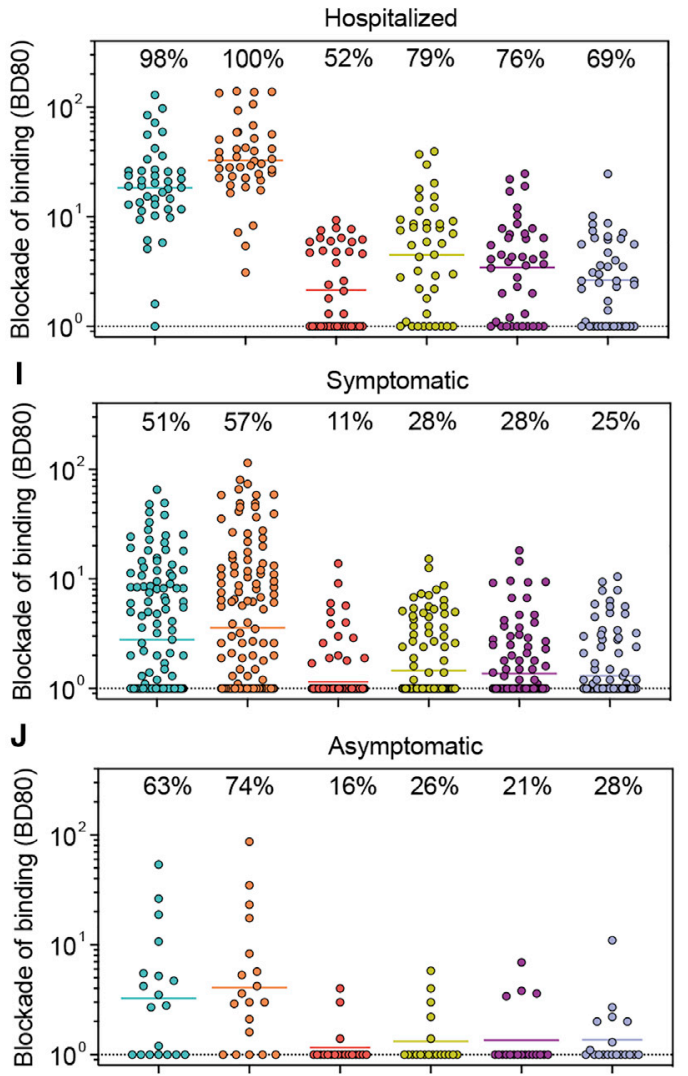

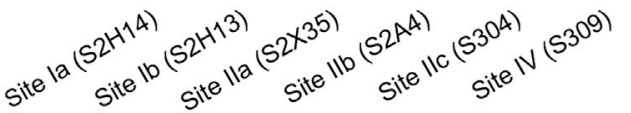
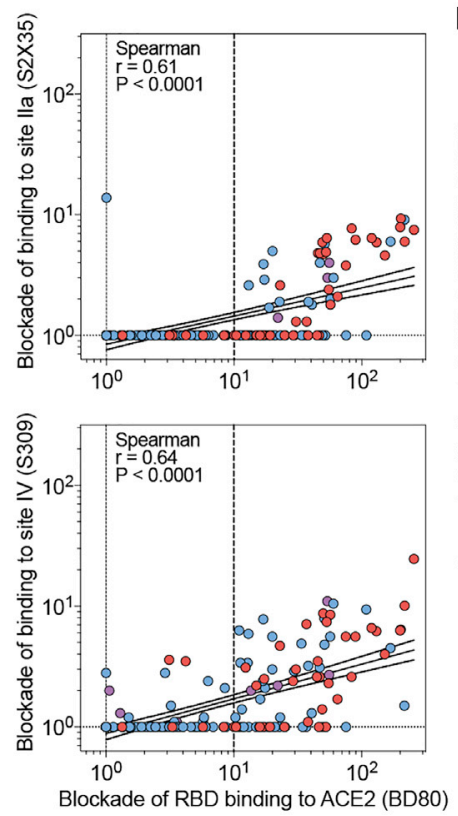

L

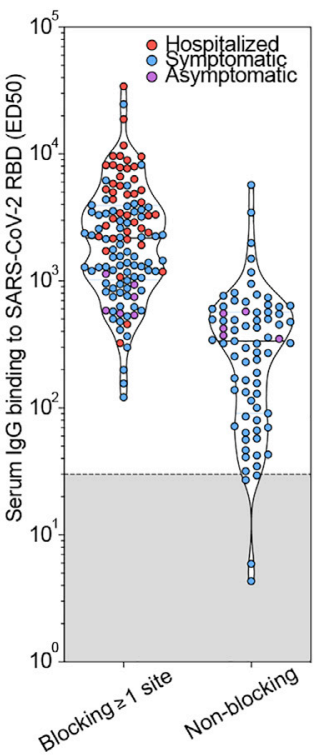


we found that binding of all mAbs to the $S$ ectodomain was dampened at $\mathrm{pH} 5.4$, except for $\mathrm{S} 309$ and $\mathrm{S} 2 \mathrm{H} 13$, whereas binding of all these mAbs to the free RBD was not affected at pH 5.4 (Figures S5A and S5B). S309 and S2H13 do not select for a specific $S$ conformation or promote $S_{1}$ shedding, which are specific features of site la- and site II-targeted mAbs (Figure S3E). Based on these data, we hypothesize that high-density binding of S309 or $\mathrm{S} 2 \mathrm{H} 13$ to multiple $\mathrm{S}$ conformations may explain their unique ability to trigger Fc-mediated effector functions efficiently among the panel of mAbs tested.

To characterize the fine specificity of $A b$ responses to the RBD in SARS-CoV-2 infected individuals, we developed a quantitative blockade-of-binding assay using the six structurally defined $m A b s$ as probes for the corresponding antigenic sites (Figures 7B-7G). Abs against sites la and Ib were found at high titers in hospitalized donors and in a fraction of non-hospitalized symptomatic and asymptomatic subjects and correlated with the titer of Abs blocking binding of the RBD to ACE2 (Figures $7 \mathrm{H}-7 \mathrm{~K}$ ). The serological response to the other RBD antigenic sites was overall lower (or null) but showed distinct signatures in different individuals. In particular, we did not detect Abs to any RBD sites in $22 \%$ of non-hospitalized individuals. Although we cannot rule out the possible existence of additional antigenic sites, the most plausible explanation is that these individuals possess low levels of RBD-specific Abs (Figures 7K and 7L), putatively with low avidity, compounding their detection in the blockade-of-binding assay. In addition, the overall waning of the total anti-RBD Abs was paralleled by a similar decay of Abs directed to each RBD site (Data S4). Collectively, our results demonstrate that the SARS-CoV-2 RBD is the main target of neutralizing Abs and that sites la and $\mathrm{lb}$ are prime antigenic sites.

\section{DISCUSSION}

This study provides an extensive analysis of $A b$ responses to SARS-CoV-2 S, RBD, and N in more than 600 SARS-CoV-2-infected individuals with different clinical outcomes. Collectively, our data define the immunodominance of the RBD and highlight qualitative and quantitative differences in the serological response of different individuals. The SARS-CoV-2 RBD is immunodominant in terms of total Abs elicited and is the target of $90 \%$ of the neutralizing activity present in the sera or plasma of most individuals evaluated. The remaining neutralizing activity observed in certain individuals may be accounted for by Abs targeting domain A (Chi et al., 2020), quaternary epitopes on the $S$ trimer or the $S_{2}$ subunit (Liu et al., 2020a). The observed SARS-CoV-2 RBD immunodominance may be related to its low level of glycosylation, as compared to the rest of the S protein, and to its higher accessibility on the surface of virions and virus-infected cells, which is further enhanced by RBD opening and exposure of cryptic sites (Ke et al., 2020; Turoňová et al., 2020; Walls et al., 2020; Walls et al., 2016b; 2019; Watanabe et al., 2020; Wrapp et al., 2020). The RBD immunodominance might also result from the release of the $S_{1}$ subunit either spontaneously or promoted by ACE2 and triggering antibodies (Huo et al., 2020; Song et al., 2018; Walls et al., 2019; Wec et al., 2020; Yuan et al., 2017). Immuno-complexes of Abs and dissociated $S_{1}$ subunits may engage $\mathrm{Fc} \gamma$ receptors and the complement, promoting presentation by dendritic cells and follicular dendritic cells, respectively.

Two common features found in all the disease groups analyzed are the heterogeneity of $\mathrm{Ab}$ responses and the decline of specific Ab titers over time. In the different groups analyzed, we found that average Ab titers were proportional to disease severity with hospitalized patients possessing higher Ab titers than non-hospitalized subjects. However, there was broad distribution of $A b$ titers within each group as illustrated by the fact that some asymptomatic individuals developed high levels of SARSCoV-2 S binding and neutralizing Abs. Interestingly, a similar variability (approximately two orders of magnitude) was recently reported in healthy volunteers vaccinated with ChAdOx1 nCoV19 vaccine (Folegatti et al., 2020). These differences might be due to different levels of antigenic exposure and possibly to pre-existing humoral immunity (Grifoni et al., 2020; $\mathrm{Ng}$ et al., 2020) as well as other factors, such as immune status, co-morbidities, or antigenic load.

We observed waning of IgG titers to RBD with an approximate half-life of 49 days in the individuals that we could follow for a period of approximately 4 months, a finding that is consistent with a recent study (Seow et al., 2020). The half-life determined in our study is longer than the average IgG half-life, which is about 20 days, indicating sustained Ab production by short-lived or long-lived plasma cells. If stable, this kinetics of decay would lead to a potential $99 \%$ reduction of RBD-specific IgG titers a year after infection, although a more precise determination of the long-term decay will require follow-up studies. However, the overall decay of RBD-specific Abs was accompanied by an increase in Ab titers blocking ACE2 binding in several individuals and by an increase in the avidity of RBD-specific IgG Abs, consistent with an ongoing affinity maturation of the $\mathrm{Ab}$ response to SARS-CoV-2 S. These findings highlight the importance of using both binding and functional assays to evaluate the Ab responses induced by vaccines.

The abundant IgG and IgA responses to SARS-CoV-2 $\mathrm{N}$ is reminiscent of the $A b$ responses to other internal viral proteins

Figure 7. Structure-Guided High-Resolution Serology

(A) Composite model of the SARS-CoV-2 S trimer with three open RBDs viewed along two orientations with all six mAbs used for competition ELISA shown bound to one RBD.

(B-G) Epitopes recognized by each mAb are shown on the surface of the RBD for S2H14 (teal, B), S2H13 (orange, C), S2X35 (red, D), S2A4 (yellow, E), S304 (magenta, F), and S309 (purple, G). The glycan at position N343 is rendered as blue spheres and the RBM is shown as a black outline.

$(\mathrm{H}-\mathrm{J})$ Competition ELISA (blockade-of-binding) between individual mAbs and sera or plasma from hospitalized (H), symptomatic (I), and asymptomatic (J) COVID19 convalescent subjects. Each plot shows the magnitude of inhibition of binding to immobilized RBD in the presence of each mAb, expressed as reciprocal sera or plasma dilution blocking $80 \%$ of the maximum binding response.

(K) Correlation analysis of titers of serum Abs blocking RBD binding to ACE2 and Abs blocking each of the six probe mAbs.

(L) Comparison of RBD-specific IgG titers between sera containing Ab blocking at least one probe mAb and sera that do not contain Ab blocking any of the six probe mAbs. 
such as p24 in HIV-1 and HBcAg in HBV and is most likely due to the abundant release of $\mathrm{N}$ protein from killed infected cells and/ or disassembled virions (Gimson et al., 1983; Lindhardt et al., 1989). The lack of detectable IgM responses to $N$ is a surprising finding and may be related to either activation of a cross-reactive memory response to coronavirus $\mathrm{N}$ proteins or rapid classswitching due to the highly stimulatory capacity of antigens that are associated with RNA, which is a strong TLR7 agonist (Lund et al., 2004).

We used information gained from six different mAb structures to develop a high-resolution serological epitope-mapping approach and define a blueprint of polyclonal $A b$ responses to the SARS-CoV-2 RBD. These data present a quantitative antigenic map of the epitopes targeted by neutralizing mAbs that explains immunodominance, neutralization properties, and activation of effector functions. We found that the RBM is immunodominant and comprises two partially overlapping antigenic sites (la and lb defined by mAbs $\mathrm{S} 2 \mathrm{H} 14$ and $\mathrm{S} 2 \mathrm{H} 13$, respectively) targeted by neutralizing Abs inhibiting ACE2 attachment. Whereas site la coincides with the ACE2 binding site and is accessible only in the open S conformation, site $\mathrm{Ib}$ is also exposed in the closed $\mathrm{S}$ conformation and is targeted by Abs with both neutralizing activity and effector function. In contrast, the remaining RBD sites, Ila, Ilb, Ilc, and IV, are subdominant and generate lower and variable $A b$ responses in different individuals. The immunodominance of sites la and $\mathrm{lb}$ may be related to their greater accessibility compared to sites $\mathrm{Ila}$, Ilb, and Ilc, as the latter epitopes become exposed only after opening of two RBDs, which is a rare event (Ke et al., 2020; Walls et al., 2020; Wrapp et al., 2020). Although site IV is accessible in both open and closed S conformations, its subdominance may result from the masking effect of a conserved glycan (at position N343) within this antigenic site (Pinto et al., 2020). Overall, the observed increase in $A b$ titers blocking RBD attachment to ACE2 in the context of waning Ab titers is consistent with the putative production of higher-affinity RBD-specific Abs, most of them targeting sites la and lb.

Numerous amino acid substitutions have been detected in the $\mathrm{RBD}$, with several of them found in the RBM (including the $\mathrm{S} 2 \mathrm{H} 13$ and $\mathrm{S} 2 \mathrm{H} 14$ epitopes), of the 74,000 SARS-CoV-2 isolates available to date in the GISAID database (Elbe and Buckland-Merrett, 2017). As sites la and Ib within the RBM are prime targets of neutralizing Abs, we suggest that mutations leading to viral escape from mAb neutralization might have been selected, possibly during prolonged infections, eventually resulting in antigenic drift similar to influenza A viruses (Hensley et al., 2009). This is supported by the fact that naturally occurring RBD mutations were recently associated with escape from $\mathrm{mAb}$ binding and with reduced recognition by immune sera (Li et al., 2020b).

Fc-mediated effector functions are key antiviral pathways in vivo that can be profoundly affected by the epitope specificities of the mAbs (DiLillo et al., 2014; Hessell et al., 2007). The finding that only $\mathrm{S} 309$ and $\mathrm{S} 2 \mathrm{H} 13$ (out of the six mAbs evaluated in this study) efficiently activated effector functions underscores the importance of the orientation and distance of the Fc fragment from the plasma membrane (DiLillo et al., 2016; Pinto et al., 2020; Tang et al., 2019) and the requirement for a high-density binding of $\mathrm{mAbs}$ for efficient $\mathrm{Fc} \gamma$ receptors cross-linking and engage- ment of the hexameric C1q. Both $\mathrm{S} 309$ and $\mathrm{S} 2 \mathrm{H} 13$ mAbs recognize epitopes accessible independently of the RBD conformation and are therefore expected to reach high occupancy on $\mathrm{S}$ trimers (Ortiz et al., 2016). Instead, Abs targeting site la and site II promote shedding of the $S_{1}$ subunit, which may limit their ability to trigger effector functions. Additional studies are needed to address the possibility of bivalent IgG binding within an $S$ trimer and the putative role of cross-linking neighboring $S$ proteins on virions or between virions to further our understanding of the mechanisms of viral neutralization.

We isolated several distinct neutralizing mAbs from plasma cells of infected donors as early as 2 weeks after onset of symptoms. These mAbs have very few somatic hypermutations, indicating that a rapid response can rely on the naive $B$ cell repertoire. These findings are consistent with a previous study of the potent MERS-CoV m336 mAb, which harbors a single somatic mutation in the heavy chain (Ying et al., 2015) as well as with recent COVID19 reports (Ju et al., 2020; Seydoux et al., 2020). These findings imply that extensive somatic hypermutation is not necessary to achieve neutralization of SARS-CoV-2 and other coronaviruses and that similar Abs might be easily elicited through vaccination. Given the immunodominance of the RBD for the elicitation of neutralizing Abs, we suggest that SARS-CoV-2 RBD-based immunogens, potentially engineered for multivalency, bear the promise of eliciting potent neutralizing $\mathrm{Ab}$ responses and represent an attractive target for rational vaccine design.

\section{STAR $\star$ METHODS}

Detailed methods are provided in the online version of this paper and include the following:

- KEY RESOURCES TABLE

- RESOURCE AVAILABILITY

O Lead Contact

O Materials Availability

Data and Code Availability

- EXPERIMENTAL MODEL AND SUBJECT DETAILS

O Cell lines

O Sample donors and collection

- METHOD DETAILS

O Isolation of peripheral blood mononuclear cells (PBMCs), plasma and sera

O Ab discovery and recombinant expression

O Recombinant glycoprotein production

○ Enzyme-linked immunosorbent assay (ELISA)

O Depletion of RBD-specific Abs from plasma or serum samples

O Pseudovirus neutralization assays

O Blockade of RBD binding to ACE2

Blockade of $m A b$ binding to RBD

Cell-surface $\mathrm{mAb}$-mediated $\mathrm{S}_{1}$ shedding

Western blot

Measurement of effector functions

O Affinity and avidity determination by surface plasmon resonance (SPR)

O Competition experiments using biolayer interferometry (Octet) 


\author{
O Crystallization, data collection, structure determination \\ and analysis \\ O CryoEM sample preparation, data collection and data \\ processing \\ O CryoEM model building and analysis \\ $\bigcirc$ Longitudinal Mixed-Effects Modeling \\ - QUANTIFICATION AND STATISTICAL ANALYSIS
}

\section{SUPPLEMENTAL INFORMATION}

Supplemental Information can be found online at https://doi.org/10.1016/j. cell.2020.09.037.

\section{ACKNOWLEDGMENTS}

This study was supported by the National Institute of General Medical Sciences (R01GM120553, D.V.), the National Institute of Allergy and Infectious Diseases (DP1Al158186 and HHSN272201700059C, D.V.), a Pew Biomedical Scholars Award (D.V.), an Investigators in the Pathogenesis of Infectious Disease Award from the Burroughs Wellcome Fund (D.V.), Fast Grants (D.V.), the University of Washington Arnold and Mabel Beckman cryo-EM center, the Pasteur Institute (M.A.T.), and beamline 4.2.2 and 5.0.2 at the Advanced Light Source at Lawrence Berkeley National Laboratory. Both beamlines are supported in part by the National Institutes of Health, National Institute of General Medical Sciences (grant P30 GM124169-01). The Advanced Light Source is a US Department of Energy Office of Science User Facility under Contract No. DE-AC02-05CH11231. F.S. is supported by the Henry Krenter Foundation. We would like to thank Isaac Hoffman for his help with refinement of the S304-S309-S2H14-RBD crystal structure. We would like to thank Marcel Meury for assistance with protein production and Josh Dillen and Cindy $\mathrm{Ng}$ for assistance with protein production and crystallization. We acknowledge Tatiana Terrot (Ente Ospedaliero Cantonale, Lugano, Switzerland) as well as Marco Schiuma and Alice Covizzi (Luigi Sacco Hospital) for filling the database of the Ticino healthcare workers and Luigi Sacco Hospital cohorts, respectively. We are grateful to Joel Quispe and Quinton Beedle for assistance with data collection. We thank Dr. Alice Covizzi, Dr. Marco Schiuma, and Prof. Massimo Galli, Director of the Unit for their help with patient sample collection.

\section{AUTHOR CONTRIBUTIONS}

Experiment Design, L.P., M.A.T., G.S., D.C., and D.V.; Donors' Recruitment and Sample Collection, G.L.P., A.F.P., M. Biggiogero, C.H.-D., M.S., D.H., V.L., A.C., E.B., L.E., P.F., C.G., A.R., and M.T.; Sample Processing, M. Beltramello, C.S.-F., D.P., F.M., S. Jovic, B.F.R., and M.T.; Serological Assays, M. Beltramello, C.S.-F., D.P., F.Z., J.B., A.P., A.D.M., F.M., S. Jovic, and B.F.R.; Expressed and Purified Proteins, M.A.T., N.C., A.C.W., J.E.B., E.C., N.S., and S. Jaconi; Isolation and Characterization of mAbs:, E.C., M.S.P., M. Beltramello, D.P., B.G., A.M., F.Z., A.P., and A.D.M.; SPR Binding Assays, L.E.R.; Models of Serum Antibody Decay; S.V.G. and F.J.; Cryo-EM Data Collection, Processing, and Model Building, Y.-J.P., M.A.T., A.C.W, O.J.A. and D.V. Crystallization, X-Ray Crystallography Data Collection, Processing, and Model Building, Y.-J.P., M.A.T., N.C., J.C.N., G.S., and D.V.; Data Analysis, L.P., Y.-J.P., M.A.T., A.C.W., G.P., E.A., G.S., F.S., K.F., D.C., and D.V.; Manuscript Writing, L.P., H.W.V., A.L., D.C., and D.V.; Supervision, L.P., M.A.T., G.S., F.S., K.F., H.W.V., A.L., D.C., and D.V.; Funding Acquisition: D.V.

\section{DECLARATION OF INTERESTS}

L.P., N.C., M. Beltramello, C.S.-F., D.P., L.E.R., F.Z., N.S., J.B., A.P., S. Jaconi, B.G., A.M., A.D.M., M.S.P., E.C., S.V.G., F.J., C.H.-D., M.S., D.H., G.S., K.F., H.W.V., A.L., and D.C. are employees of Vir Biotechnology Inc. and may hold shares in Vir Biotechnology Inc. D.C. is currently listed as an inventor on multiple patent applications, which disclose the subject matter described in this manuscript. The Veesler laboratory has received a sponsored research agree- ment from Vir Biotechnology Inc. The other authors declare no competing interests.

Received: August 3, 2020

Revised: August 28, 2020

Accepted: September 11, 2020

Published: September 16, 2020

\section{REFERENCES}

Agirre, J., Iglesias-Fernández, J., Rovira, C., Davies, G.J., Wilson, K.S., and Cowtan, K.D. (2015). Privateer: software for the conformational validation of carbohydrate structures. Nat. Struct. Mol. Biol. 22, 833-834.

Alshukairi, A.N., Khalid, I., Ahmed, W.A., Dada, A.M., Bayumi, D.T., Malic, L.S., Althawadi, S., Ignacio, K., Alsalmi, H.S., Al-Abdely, H.M., et al. (2016). Antibody Response and Disease Severity in Healthcare Worker MERS Survivors. Emerg. Infect. Dis. 22, 1113-1115.

Alsoussi, W.B., Turner, J.S., Case, J.B., Zhao, H., Schmitz, A.J., Zhou, J.Q., Chen, R.E., Lei, T., Rizk, A.A., McIntire, K.M., et al. (2020). A Potently Neutralizing Antibody Protects Mice against SARS-CoV-2 Infection. J Immunol 205, ji2000583.

Barad, B.A., Echols, N., Wang, R.Y., Cheng, Y., DiMaio, F., Adams, P.D., and Fraser, J.S. (2015). EMRinger: side chain-directed model and map validation for 3D cryo-electron microscopy. Nat. Methods 12, 943-946.

Barnes, C.O., West, A.P., Huey-Tubman, K.E., Hoffmann, M.A.G., Sharaf, N.G., Hoffman, P.R., Koranda, N., Gristick, H.B., Gaebler, C., Muecksch, F., et al. (2020). Structures of Human Antibodies Bound to SARS-CoV-2 Spike Reveal Common Epitopes and Recurrent Features of Antibodies. Cell 182, 828-842.16.

Battye, T.G., Kontogiannis, L., Johnson, O., Powell, H.R., and Leslie, A.G. (2011). iMOSFLM: a new graphical interface for diffraction-image processing with MOSFLM. Acta Crystallogr. D Biol. Crystallogr. 67, 271-281.

Baum, A., Fulton, B.O., Wloga, E., Copin, R., Pascal, K.E., Russo, V., Giordano, S., Lanza, K., Negron, N., Ni, M., et al. (2020). Antibody cocktail to SARS-CoV2 spike protein prevents rapid mutational escape seen with individual antibodies. Science 369, 1014-1018.

Brouwer, P.J.M., Caniels, T.G., van der Straten, K., Snitselaar, J.L., Aldon, Y. Bangaru, S., Torres, J.L., Okba, N.M.A., Claireaux, M., Kerster, G., et al. (2020). Potent neutralizing antibodies from COVID-19 patients define multiple targets of vulnerability. Science 369, 643-650.

Callow, K.A. (1985). Effect of specific humoral immunity and some non-specific factors on resistance of volunteers to respiratory coronavirus infection. J. Hyg. (Lond.) 95, 173-189.

Callow, K.A., Parry, H.F., Sergeant, M., and Tyrrell, D.A. (1990). The time course of the immune response to experimental coronavirus infection of man. Epidemiol. Infect. 105, 435-446.

Cao, W.C., Liu, W., Zhang, P.H., Zhang, F., and Richardus, J.H. (2007). Disappearance of antibodies to SARS-associated coronavirus after recovery. N. Engl. J. Med. 357, 1162-1163.

Cardone, G., Heymann, J.B., and Steven, A.C. (2013). One number does not fit all: mapping local variations in resolution in cryo-EM reconstructions. J. Struct. Biol. 184, 226-236.

Chen, V.B., Arendall, W.B., 3rd, Headd, J.J., Keedy, D.A., Immormino, R.M. Kapral, G.J., Murray, L.W., Richardson, J.S., and Richardson, D.C. (2010). MolProbity: all-atom structure validation for macromolecular crystallography. Acta Crystallogr. D Biol. Crystallogr. 66, 12-21.

Chen, S., McMullan, G., Faruqi, A.R., Murshudov, G.N., Short, J.M., Scheres, S.H., and Henderson, R. (2013). High-resolution noise substitution to measure overfitting and validate resolution in 3D structure determination by single particle electron cryomicroscopy. Ultramicroscopy 135, 24-35.

Chi, X., Yan, R., Zhang, J., Zhang, G., Zhang, Y., Hao, M., Zhang, Z., Fan, P. Dong, Y., Yang, Y., et al. (2020). A neutralizing human antibody binds to the $\mathrm{N}$-terminal domain of the Spike protein of SARS-CoV-2. Science 369, 650-655. 
Corti, D., Voss, J., Gamblin, S.J., Codoni, G., Macagno, A., Jarrossay, D., Vachieri, S.G., Pinna, D., Minola, A., Vanzetta, F., et al. (2011). A neutralizing antibody selected from plasma cells that binds to group 1 and group 2 influenza $A$ hemagglutinins. Science 333, 850-856.

Corti, D., Zhao, J., Pedotti, M., Simonelli, L., Agnihothram, S., Fett, C., Fernandez-Rodriguez, B., Foglierini, M., Agatic, G., Vanzetta, F., et al. (2015). Prophylactic and postexposure efficacy of a potent human monoclonal antibody against MERS coronavirus. Proc. Natl. Acad. Sci. USA 112, 10473-10478.

de Wit, E., Feldmann, F., Horne, E., Okumura, A., Cameroni, E., Haddock, E., Saturday, G., Scott, D., Gopal, R., Zambon, M., et al. (2019). Prophylactic efficacy of a human monoclonal antibody against MERS-CoV in the common marmoset. Antiviral Res. 163, 70-74.

DiLillo, D.J., and Ravetch, J.V. (2015). Differential Fc-Receptor Engagement Drives an Anti-tumor Vaccinal Effect. Cell 161, 1035-1045.

DiLillo, D.J., Tan, G.S., Palese, P., and Ravetch, J.V. (2014). Broadly neutralizing hemagglutinin stalk-specific antibodies require $\mathrm{F}_{\mathrm{c}} \gamma \mathrm{R}$ interactions for protection against influenza virus in vivo. Nat. Med. 20, 143-151.

DiLillo, D.J., Palese, P., Wilson, P.C., and Ravetch, J.V. (2016). Broadly neutralizing anti-influenza antibodies require $F_{c}$ receptor engagement for in vivo protection. J. Clin. Invest. 126, 605-610.

Drosten, C., Meyer, B., Müller, M.A., Corman, V.M., Al-Masri, M., Hossain, R., Madani, H., Sieberg, A., Bosch, B.J., Lattwein, E., et al. (2014). Transmission of MERS-coronavirus in household contacts. N. Engl. J. Med. 371, 828-835.

Duan, K., Liu, B., Li, C., Zhang, H., Yu, T., Qu, J., Zhou, M., Chen, L., Meng, S., $\mathrm{Hu}, \mathrm{Y}$., et al. (2020). Effectiveness of convalescent plasma therapy in severe COVID-19 patients. Proc. Natl. Acad. Sci. USA 117, 9490-9496.

Edridge, A.W., Kaczorowska, J.M., Hoste, A.C., Bakker, M., Klein, M., Jebbink, M.F., Matser, A., Kinsella, C., Rueda, P., Prins, M., et al. (2020). Coronavirus protective immunity is short-lasting. medRxiv. https://doi.org/10.1101/ 2020.05.11.20086439.

Elbe, S., and Buckland-Merrett, G. (2017). Data, disease and diplomacy: GlSAID's innovative contribution to global health. Glob Chall 1, 33-46.

Emsley, P., Lohkamp, B., Scott, W.G., and Cowtan, K. (2010). Features and development of Coot. Acta Crystallogr. D Biol. Crystallogr. 66, 486-501.

Evans, P.R., and Murshudov, G.N. (2013). How good are my data and what is the resolution? Acta Crystallogr. D Biol. Crystallogr. 69, 1204-1214.

Folegatti, P.M., Ewer, K.J., Aley, P.K., Angus, B., Becker, S., Belij-Rammerstorfer, S., Bellamy, D., Bibi, S., Bittaye, M., Clutterbuck, E.A., et al. (2020). Safety and immunogenicity of the ChAdOx1 nCoV-19 vaccine against SARS-CoV-2: a preliminary report of a phase $1 / 2$, single-blind, randomised controlled trial. Lancet 396, 467-478.

Frenz, B., Rämisch, S., Borst, A.J., Walls, A.C., Adolf-Bryfogle, J., Schief, W.R., Veesler, D., and DiMaio, F. (2019). Automatically Fixing Errors in Glycoprotein Structures with Rosetta. Structure 27, 134-139.e3.

Gimson, A.E., Tedder, R.S., White, Y.S., Eddleston, A.L., and Williams, R. (1983). Serological markers in fulminant hepatitis B. Gut 24, 615-617.

Goddard, T.D., Huang, C.C., Meng, E.C., Pettersen, E.F., Couch, G.S., Morris, J.H., and Ferrin, T.E. (2018). UCSF ChimeraX: Meeting modern challenges in visualization and analysis. Protein Sci. 27, 14-25.

Grifoni, A., Weiskopf, D., Ramirez, S.I., Mateus, J., Dan, J.M., Moderbacher, C.R., Rawlings, S.A., Sutherland, A., Premkumar, L., Jadi, R.S., et al. (2020). Targets of T Cell Responses to SARS-CoV-2 Coronavirus in Humans with COVID-19 Disease and Unexposed Individuals. Cell 181, 1489-1501.e15.

Guo, X., Guo, Z., Duan, C., Chen, Z., Wang, G., Lu, Y., Li, M., and Lu, J. (2020). Long-Term Persistence of IgG Antibodies in SARS-CoV Infected Healthcare Workers. medRxiv. https://doi.org/10.1101/2020.02.12.20021386.

He, W., Chen, C.J., Mullarkey, C.E., Hamilton, J.R., Wong, C.K., Leon, P.E., Uccellini, M.B., Chromikova, V., Henry, C., Hoffman, K.W., et al. (2017). Alveolar macrophages are critical for broadly-reactive antibody-mediated protection against influenza A virus in mice. Nat. Commun. 8, 846.

Hensley, S.E., Das, S.R., Bailey, A.L., Schmidt, L.M., Hickman, H.D., Jayaraman, A., Viswanathan, K., Raman, R., Sasisekharan, R., Bennink, J.R., and
Yewdell, J.W. (2009). Hemagglutinin receptor binding avidity drives influenza A virus antigenic drift. Science 326, 734-736.

Hessell, A.J., Hangartner, L., Hunter, M., Havenith, C.E., Beurskens, F.J., Bakker, J.M., Lanigan, C.M., Landucci, G., Forthal, D.N., Parren, P.W., et al. (2007). Fc receptor but not complement binding is important in antibody protection against HIV. Nature 449, 101-104.

Hoffmann, M., Kleine-Weber, H., and Pöhlmann, S. (2020a). A Multibasic Cleavage Site in the Spike Protein of SARS-CoV-2 Is Essential for Infection of Human Lung Cells. Mol. Cell 78, 779-784.e5.

Hoffmann, M., Kleine-Weber, H., Schroeder, S., Krüger, N., Herrler, T., Erichsen, S., Schiergens, T.S., Herrler, G., Wu, N.H., Nitsche, A., et al. (2020b). SARS-CoV-2 Cell Entry Depends on ACE2 and TMPRSS2 and Is Blocked by a Clinically Proven Protease Inhibitor. Cell 181, 271-280.e8.

Huo, J., Zhao, Y., Ren, J., Zhou, D., Duyvesteyn, H.M.E., Ginn, H.M., Carrique, L., Malinauskas, T., Ruza, R.R., Shah, P.N.M., et al. (2020). Neutralization of SARS-CoV-2 by destruction of the prefusion Spike. Cell Host Microbe 28, 445-454.e6.

Johnson, R.F., Bagci, U., Keith, L., Tang, X., Mollura, D.J., Zeitlin, L., Qin, J., Huzella, L., Bartos, C.J., Bohorova, N., et al. (2016). 3B11-N, a monoclonal antibody against MERS-CoV, reduces lung pathology in rhesus monkeys following intratracheal inoculation of MERS-CoV Jordan-n3/2012. Virology 490, 49-58.

Joyce, M.G., Sankhala, R.S., Chen, W.-H., Choe, M., Bai, H., Hajduczki, A., Yan, L., Sterling, S.L., Peterson, C.E., Green, E.C., et al. (2020). A Cryptic Site of Vulnerability on the Receptor Binding Domain of the SARS-CoV-2 Spike Glycoprotein. bioRxiv. https://doi.org/10.1101/2020.03.15.992883.

Ju, B., Zhang, Q., Ge, X., Wang, R., Yu, J., Shan, S., Zhou, B., Song, S., Tang, X., Yu, J., et al. (2020). Potent human neutralizing antibodies elicited by SARSCoV-2 infection. bioRxiv. https://doi.org/10.1101/2020.03.21.990770.

Kabsch, W. (2010). XDS. Acta Crystallogr. D Biol. Crystallogr. 66, 125-132.

Katoh, K., Rozewicki, J., and Yamada, K.D. (2019). MAFFT online service: multiple sequence alignment, interactive sequence choice and visualization. Briefings in Bioinformatics 20, 1160-1166.

Ke, Z., Oton, J., Qu, K., Cortese, M., Zila, V., McKeane, L., Nakane, T., Zivanov, J., Neufeldt, C.J., Cerikan, B., et al. (2020). Structures and distributions of SARS-CoV-2 spike proteins on intact virions. Nature. https://doi.org/10. 1038/s41586-020-2665-2.

Kirchdoerfer, R.N., Wang, N., Pallesen, J., Wrapp, D., Turner, H.L., Cottrell, C.A., Corbett, K.S., Graham, B.S., McLellan, J.S., and Ward, A.B. (2018). Stabilized coronavirus spikes are resistant to conformational changes induced by receptor recognition or proteolysis. Sci. Rep. 8, 15701.

Lan, J., Ge, J., Yu, J., Shan, S., Zhou, H., Fan, S., Zhang, Q., Shi, X., Wang, Q., Zhang, L., and Wang, X. (2020). Structure of the SARS-CoV-2 spike receptorbinding domain bound to the ACE2 receptor. Nature 581, 215-220.

Lau, S.Y., Wang, P., Mok, B.W., Zhang, A.J., Chu, H., Lee, A.C., Deng, S., Chen, P., Chan, K.H., Song, W., et al. (2020). Attenuated SARS-CoV-2 variants with deletions at the S1/S2 junction. Emerg. Microbes Infect. 9, 837-842.

Letko, M., Marzi, A., and Munster, V. (2020). Functional assessment of cell entry and receptor usage for SARS-CoV-2 and other lineage B betacoronaviruses. Nat. Microbiol. 5, 562-569.

Li, L., Zhang, W., Hu, Y., Tong, X., Zheng, S., Yang, J., Kong, Y., Ren, L., Wei, Q., Mei, H., et al. (2020a). Effect of Convalescent Plasma Therapy on Time to Clinical Improvement in Patients With Severe and Life-threatening COVID-19: A Randomized Clinical Trial. JAMA 324, 460-470.

Li, Q., Wu, J., Nie, J., Zhang, L., Hao, H., Liu, S., Zhao, C., Zhang, Q., Liu, H., Nie, L., et al. (2020b). The impact of mutations in SARS-CoV-2 spike on viral infectivity and antigenicity. Cell 182, 1284-1294.e9.

Liebschner, D., Afonine, P.V., Baker, M.L., Bunkóczi, G., Chen, V.B., Croll, T.I., Hintze, B., Hung, L.W., Jain, S., McCoy, A.J., et al. (2019). Macromolecular structure determination using $\mathrm{X}$-rays, neutrons and electrons: recent developments in Phenix. Acta Crystallogr. D Struct. Biol. 75, 861-877.

Lindhardt, B.O., Gerstoft, J., Hofmann, B., Pallesen, G., Mathiesen, L., Dickmeiss, E., and Ulrich, K. (1989). Antibodies against the major core protein 
p24 of human immunodeficiency virus: relation to immunological, clinical and prognostic findings. Eur. J. Clin. Microbiol. Infect. Dis. 8, 614-619.

Liu, L., Wang, P., Nair, M.S., Yu, J., Rapp, M., Wang, Q., Luo, Y., Chan, J.F. Sahi, V., Figueroa, A., et al. (2020a). Potent Neutralizing Monoclonal Antibodies Directed to Multiple Epitopes on the SARS-CoV-2 Spike. bioRxiv. https://doi. org/10.1101/2020.06.17.153486.

Liu, S.T.H., Lin, H.-M., Baine, I., Wajnberg, A., Gumprecht, J.P., Rahman, F., Rodriguez, D., Tandon, P., Bassily-Marcus, A., Bander, J., et al. (2020b). Convalescent plasma treatment of severe COVID-19: A matched control study. medRxiv. https://doi.org/10.1101/2020.05.20.20102236.

Long, Q.X., Tang, X.J., Shi, Q.L., Li, Q., Deng, H.J., Yuan, J., Hu, J.L., Xu, W., Zhang, Y., LV, F.J., et al. (2020). Clinical and immunological assessment of asymptomatic SARS-CoV-2 infections. Nat. Med. 26, 1200-1204.

Luchsinger, L.L., Ransegnola, B., Jin, D., Muecksch, F., Weisblum, Y., Bao, W., George, P.J., Rodriguez, M., Tricoche, N., Schmidt, F., et al. (2020). Serological Analysis of New York City COVID19 Convalescent Plasma Donors. medRxiv. https://doi.org/10.1101/2020.06.08.20124792.

Lund, J.M., Alexopoulou, L., Sato, A., Karow, M., Adams, N.C., Gale, N.W. Iwasaki, A., and Flavell, R.A. (2004). Recognition of single-stranded RNA viruses by Toll-like receptor 7. Proc. Natl. Acad. Sci. USA 101, 5598-5603.

McCoy, A.J., Grosse-Kunstleve, R.W., Adams, P.D., Winn, M.D., Storoni, L.C., and Read, R.J. (2007). Phaser crystallographic software. J. Appl. Cryst. 40, 658-674.

Menachery, V.D., Yount, B.L., Jr., Sims, A.C., Debbink, K., Agnihothram, S.S. Gralinski, L.E., Graham, R.L., Scobey, T., Plante, J.A., Royal, S.R., et al. (2016) SARS-like WIV1-CoV poised for human emergence. Proc. Natl. Acad. Sci. USA 113, 3048-3053.

Millet, J.K., and Whittaker, G.R. (2015). Host cell proteases: Critical determinants of coronavirus tropism and pathogenesis. Virus Res. 202, 120-134.

Murshudov, G.N., Skubák, P., Lebedev, A.A., Pannu, N.S., Steiner, R.A., Nicholls, R.A., Winn, M.D., Long, F., and Vagin, A.A. (2011). REFMAC5 for the refinement of macromolecular crystal structures. Acta Crystallogr. D Biol. Crystallogr. 67, 355-367.

Needleman, S.B., and Wunsch, C.D. (1970). A general method applicable to the search for similarities in the amino acid sequence of two proteins. Journal of Molecular Biology 48, 443-453.

Ng, K.W., Faulkner, N., Cornish, G.H., Rosa, A., Harvey, R., Hussain, S., Ulferts, R., Earl, C., Wrobel, A., Benton, D., et al. (2020). Pre-existing and de novohumoral immunity to SARS-CoV-2 in humans. bioRxiv. https://doi.org/10. 1101/2020.05.14.095414.

Ortiz, D.F., Lansing, J.C., Rutitzky, L., Kurtagic, E., Prod'homme, T., Choudhury, A., Washburn, N., Bhatnagar, N., Beneduce, C., Holte, K., et al. (2016). Elucidating the interplay between IgG-Fc valency and $F_{c} \gamma R$ activation for the design of immune complex inhibitors. Sci. Transl. Med. 8, 365ra158.

Ou, X., Liu, Y., Lei, X., Li, P., Mi, D., Ren, L., Guo, L., Guo, R., Chen, T., Hu, J., et al. (2020). Characterization of spike glycoprotein of SARS-CoV-2 on virus entry and its immune cross-reactivity with SARS-CoV. Nat. Commun. $11,1620$.

Pallesen, J., Wang, N., Corbett, K.S., Wrapp, D., Kirchdoerfer, R.N., Turner, H.L., Cottrell, C.A., Becker, M.M., Wang, L., Shi, W., et al. (2017). Immunogenicity and structures of a rationally designed prefusion MERS-CoV spike antigen. Proc. Natl. Acad. Sci. USA 114, E7348-E7357.

Pettersen, E.F., Goddard, T.D., Huang, C.C., Couch, G.S., Greenblatt, D.M., Meng, E.C., and Ferrin, T.E. (2004). UCSF Chimera-a visualization system for exploratory research and analysis. J. Comput. Chem. 25, 1605-1612.

Pinto, D., Park, Y.J., Beltramello, M., Walls, A.C., Tortorici, M.A., Bianchi, S., Jaconi, S., Culap, K., Zatta, F., De Marco, A., et al. (2020). Cross-neutralization of SARS-CoV-2 by a human monoclonal SARS-CoV antibody. Nature 583, 290-295

Prévost, J., Gasser, R., Beaudoin-Bussières, G., Richard, J., Duerr, R., Laumaea, A., Anand, S.P., Goyette, G., Ding, S., Medjahed, H., et al. (2020). Cross-sectional evaluation of humoral responses against SARS-CoV-2 Spike. bioRxiv. https://doi.org/10.1101/2020.06.08.140244.
Punjani, A., Rubinstein, J.L., Fleet, D.J., and Brubaker, M.A. (2017). cryoSPARC: algorithms for rapid unsupervised cryo-EM structure determination. Nat. Methods 14, 290-296.

Punjani, A., Zhang, H., and Fleet, D.J. (2019). Non-uniform refinement: Adaptive regularization improves single particle cryo-EM reconstruction. bioRxiv. https://doi.org/10.1101/2019.12.15.877092.

Robbiani, D.F., Gaebler, C., Muecksch, F., Lorenzi, J.C.C., Wang, Z., Cho, A., Agudelo, M., Barnes, C.O., Gazumyan, A., Finkin, S., et al. (2020). Convergent antibody responses to SARS-CoV-2 in convalescent individuals. Nature 584, 437-442.

Rockx, B., Corti, D., Donaldson, E., Sheahan, T., Stadler, K., Lanzavecchia, A., and Baric, R. (2008). Structural basis for potent cross-neutralizing human monoclonal antibody protection against lethal human and zoonotic severe acute respiratory syndrome coronavirus challenge. J. Virol. 82, 3220-3235.

Rogers, T.F., Zhao, F., Huang, D., Beutler, N., Burns, A., He, W.T., Limbo, O., Smith, C., Song, G., Woehl, J., et al. (2020). Isolation of potent SARS-CoV-2 neutralizing antibodies and protection from disease in a small animal model. Science 369, 956-963.

Scheres, S.H. (2012a). A Bayesian view on cryo-EM structure determination. J. Mol. Biol. 415, 406-418.

Scheres, S.H. (2012b). RELION: implementation of a Bayesian approach to cryo-EM structure determination. J. Struct. Biol. 180, 519-530.

Seow, J., Graham, C., Merrick, B., Acors, S., Steel, K.J.A., Hemmings, O. O'Bryne, A., Kouphou, N., Pickering, S., Galao, R., et al. (2020). Longitudinal evaluation and decline of antibody responses in SARS-CoV-2 infection. medRxiv. https://doi.org/10.1101/2020.07.09.20148429.

Seydoux, E., Homad, L.J., MacCamy, A.J., Parks, K.R., Hurlburt, N.K., Jennewein, M.F., Akins, N.R., Stuart, A.B., Wan, Y.-H., Feng, J., et al. (2020). Characterization of neutralizing antibodies from a SARS-CoV-2 infected individual. bioRxiv. https://doi.org/10.1101/2020.05.12.091298.

Shang, J., Ye, G., Shi, K., Wan, Y., Luo, C., Aihara, H., Geng, Q., Auerbach, A. and Li, F. (2020). Structural basis of receptor recognition by SARS-CoV-2. Nature $581,221-224$

Shen, C., Wang, Z., Zhao, F., Yang, Y., Li, J., Yuan, J., Wang, F., Li, D., Yang M., Xing, L., et al. (2020). Treatment of 5 Critically III Patients With COVID-19 With Convalescent Plasma. JAMA 323, 1582-1589.

Song, W., Gui, M., Wang, X., and Xiang, Y. (2018). Cryo-EM structure of the SARS coronavirus spike glycoprotein in complex with its host cell receptor ACE2. PLoS Pathog. 14, e1007236.

Stettler, K., Beltramello, M., Espinosa, D.A., Graham, V., Cassotta, A., Bianchi, S., Vanzetta, F., Minola, A., Jaconi, S., Mele, F., et al. (2016). Specificity, crossreactivity, and function of antibodies elicited by Zika virus infection. Science $353,823-826$.

Suloway, C., Pulokas, J., Fellmann, D., Cheng, A., Guerra, F., Quispe, J., Stagg, S., Potter, C.S., and Carragher, B. (2005). Automated molecular microscopy: the new Leginon system. J. Struct. Biol. 151, 41-60.

Tan, Y.Z., Baldwin, P.R., Davis, J.H., Williamson, J.R., Potter, C.S., Carragher, B., and Lyumkis, D. (2017). Addressing preferred specimen orientation in single-particle cryo-EM through tilting. Nat. Methods 14, 793-796.

Tan, C.W., Chia, W.N., Qin, X., Liu, P., Chen, M.I.C., Tiu, C., Hu, Z., Chen, V.C. W., Young, B.E., Sia, W.R., et al. (2020). A SARS-CoV-2 surrogate virus neutralization test based on antibody-mediated blockage of ACE2-spike protein-protein interaction. Nature Biotechnology 38, 1073-1078.

Tang, A., Chen, Z., Cox, K.S., Su, H.P., Callahan, C., Fridman, A., Zhang, L. Patel, S.B., Cejas, P.J., Swoyer, R., et al. (2019). A potent broadly neutralizing human RSV antibody targets conserved site IV of the fusion glycoprotein. Nat. Commun. 10, 4153

Tegunov, D., and Cramer, P. (2019). Real-time cryo-electron microscopy data preprocessing with Warp. Nat. Methods 16, 1146-1152.

ter Meulen, J., van den Brink, E.N., Poon, L.L., Marissen, W.E., Leung, C.S. Cox, F., Cheung, C.Y., Bakker, A.Q., Bogaards, J.A., van Deventer, E., et al. (2006). Human monoclonal antibody combination against SARS coronavirus: synergy and coverage of escape mutants. PLoS Med. 3, e237. 
Tian, X., Li, C., Huang, A., Xia, S., Lu, S., Shi, Z., Lu, L., Jiang, S., Yang, Z., Wu, Y., and Ying, T. (2020). Potent binding of 2019 novel coronavirus spike protein by a SARS coronavirus-specific human monoclonal antibody. Emerg. Microbes Infect. 9, 382-385.

Tiller, T., Meffre, E., Yurasov, S., Tsuiji, M., Nussenzweig, M.C., and Wardemann, H. (2008). Efficient generation of monoclonal antibodies from single human B cells by single cell RT-PCR and expression vector cloning. J Immunol Methods 329, 112-124.

Tortorici, M.A., and Veesler, D. (2019). Structural insights into coronavirus entry. Adv. Virus Res. 105, 93-116.

Traggiai, E., Becker, S., Subbarao, K., Kolesnikova, L., Uematsu, Y., Gismondo, M.R., Murphy, B.R., Rappuoli, R., and Lanzavecchia, A. (2004). An efficient method to make human monoclonal antibodies from memory B cells: potent neutralization of SARS coronavirus. Nat. Med. 10, 871-875.

Turoňová, B., Sikora, M., Schürmann, C., Hagen, W.J.H., Welsch, S., Blanc, F.E.C., von Bülow, S., Gecht, M., Bagola, K., Hörner, C., et al. (2020). In situ structural analysis of SARS-CoV-2 spike reveals flexibility mediated by three hinges. Science, eabd5223.

Walls, A.C., Tortorici, M.A., Bosch, B.J., Frenz, B., Rottier, P.J.M., DiMaio, F., Rey, F.A., and Veesler, D. (2016a). Cryo-electron microscopy structure of a coronavirus spike glycoprotein trimer. Nature 531, 114-117.

Walls, A.C., Tortorici, M.A., Frenz, B., Snijder, J., Li, W., Rey, F.A., DiMaio, F., Bosch, B.J., and Veesler, D. (2016b). Glycan shield and epitope masking of a coronavirus spike protein observed by cryo-electron microscopy. Nat. Struct. Mol. Biol. 23, 899-905.

Walls, A.C., Tortorici, M.A., Snijder, J., Xiong, X., Bosch, B.J., Rey, F.A., and Veesler, D. (2017). Tectonic conformational changes of a coronavirus spike glycoprotein promote membrane fusion. Proc. Natl. Acad. Sci. USA 114, $11157-11162$

Walls, A.C., Xiong, X., Park, Y.J., Tortorici, M.A., Snijder, J., Quispe, J., Cameroni, E., Gopal, R., Dai, M., Lanzavecchia, A., et al. (2019). Unexpected Receptor Functional Mimicry Elucidates Activation of Coronavirus Fusion. Cell 176, 1026-1039.e15.

Walls, A.C., Park, Y.J., Tortorici, M.A., Wall, A., McGuire, A.T., and Veesler, D. (2020). Structure, Function, and Antigenicity of the SARS-CoV-2 Spike Glycoprotein. Cell 181, 281-292.e6.

Wang, R.Y., Song, Y., Barad, B.A., Cheng, Y., Fraser, J.S., and DiMaio, F. (2016). Automated structure refinement of macromolecular assemblies from cryo-EM maps using Rosetta. eLife 5, e17219.

Wang, C., Li, W., Drabek, D., Okba, N.M.A., van Haperen, R., Osterhaus, A.D.M.E., van Kuppeveld, F.J.M., Haagmans, B.L., Grosveld, F., and Bosch, B.-J. (2020a). A human monoclonal antibody blocking SARS-CoV-2 infection. Nature Communications 11, 2251.
Wang, Q., Zhang, Y., Wu, L., Niu, S., Song, C., Zhang, Z., Lu, G., Qiao, C., Hu, Y., Yuen, K.Y., et al. (2020b). Structural and Functional Basis of SARS-CoV-2 Entry by Using Human ACE2. Cell 181, 894-904.e9.

Watanabe, Y., Allen, J.D., Wrapp, D., McLellan, J.S., and Crispin, M. (2020). Site-specific glycan analysis of the SARS-CoV-2 spike. Science 369, 330-333.

Wec, A.Z., Wrapp, D., Herbert, A.S., Maurer, D.P., Haslwanter, D., Sakharkar, M., Jangra, R.K., Dieterle, M.E., Lilov, A., Huang, D., et al. (2020). Broad neutralization of SARS-related viruses by human monoclonal antibodies. Science $369,731-736$.

Wrapp, D., Wang, N., Corbett, K.S., Goldsmith, J.A., Hsieh, C.L., Abiona, O., Graham, B.S., and McLellan, J.S. (2020). Cryo-EM structure of the 2019nCoV spike in the prefusion conformation. Science 367, 1260-1263.

Wu, Y., Wang, F., Shen, C., Peng, W., Li, D., Zhao, C., Li, Z., Li, S., Bi, Y., Yang, Y., et al. (2020). A noncompeting pair of human neutralizing antibodies block COVID-19 virus binding to its receptor ACE2. Science 368, 1274-1278.

Yan, R., Zhang, Y., Li, Y., Xia, L., Guo, Y., and Zhou, Q. (2020). Structural basis for the recognition of SARS-CoV-2 by full-length human ACE2. Science 367, 1444-1448.

Ying, T., Prabakaran, P., Du, L., Shi, W., Feng, Y., Wang, Y., Wang, L., Li, W., Jiang, S., Dimitrov, D.S., and Zhou, T. (2015). Junctional and allele-specific residues are critical for MERS-CoV neutralization by an exceptionally potent germline-like antibody. Nat. Commun. 6, 8223.

Yuan, Y., Cao, D., Zhang, Y., Ma, J., Qi, J., Wang, Q., Lu, G., Wu, Y., Yan, J., Shi, Y., et al. (2017). Cryo-EM structures of MERS-CoV and SARS-CoV spike glycoproteins reveal the dynamic receptor binding domains. Nat. Commun. 8, 15092

Yuan, M., Wu, N.C., Zhu, X., Lee, C.D., So, R.T.Y., Lv, H., Mok, C.K.P., and Wilson, I.A. (2020). A highly conserved cryptic epitope in the receptor binding domains of SARS-CoV-2 and SARS-CoV. Science 368, 630-633.

Zhou, P., Yang, X.L., Wang, X.G., Hu, B., Zhang, L., Zhang, W., Si, H.R., Zhu Y., Li, B., Huang, C.L., et al. (2020a). A pneumonia outbreak associated with a new coronavirus of probable bat origin. Nature 579, 270-273.

Zhou, T., Tsybovsky, Y., Olia, A.S., Gorman, J., Rapp, M.A., Cerutti, G., Katsamba, P.S., Nazzari, A., Schon, A., Wang, P.D., et al. (2020b). A pH-dependent switch mediates conformational masking of SARS-CoV-2 spike. bioRxiv. https://doi.org/10.1101/2020.07.04.187989.

Zivanov, J., Nakane, T., Forsberg, B.O., Kimanius, D., Hagen, W.J., Lindahl, E., and Scheres, S.H. (2018). New tools for automated high-resolution cryo-EM structure determination in RELION-3. eLife 7, e42166.

Zivanov, J., Nakane, T., and Scheres, S.H.W. (2019). A Bayesian approach to beam-induced motion correction in cryo-EM single-particle analysis. IUCrJ 6, 5-17. 


\section{STAR $\star M E T H O D S$}

\section{KEY RESOURCES TABLE}

\begin{tabular}{|c|c|c|}
\hline REAGENT or RESOURCE & SOURCE & IDENTIFIER \\
\hline \multicolumn{3}{|l|}{ Antibodies } \\
\hline S304 & (Pinto et al., 2020) & N/A \\
\hline S309 & (Pinto et al., 2020) & N/A \\
\hline S304 Fab & ATUM & N/A \\
\hline S309 Fab & ATUM & N/A \\
\hline $\mathrm{S} 2 \mathrm{H} 14 \mathrm{Fab}$ & ATUM & N/A \\
\hline $\begin{array}{l}\text { Goat } F(a b ') 2 \text { Anti-Mouse } \lg G(H+L) \text {, Human } \\
\text { ads-AP }\end{array}$ & Southern Biotech & Cat. No. $1030-04$ \\
\hline Goat Anti-Human IgG-AP & Southern Biotech & Cat. No. 2040-04 \\
\hline Goat Anti-Human IgA-AP & Southern Biotech & Cat. No. $2050-04$ \\
\hline Goat Anti-Human IgM-AP & Southern Biotech & Cat. No. $2020-04$ \\
\hline $\begin{array}{l}\text { Alexa Fluor }{ }^{\circledR} \text { 647-AffiniPure } F\left(a b^{\prime}\right) 2 \\
\text { Fragment Goat Anti-Human IgG, Fc } \gamma \\
\text { Fragment Specific }\end{array}$ & Jackson ImmunoResearch & Cat. No. $109-606-098$ \\
\hline Avi-tag Antibody, pAb, Rabbit & Genscript & Cat. No. A00674 \\
\hline \multicolumn{3}{|l|}{ Bacterial Strains and Pseudotype Viruses } \\
\hline $\mathrm{NEB} \otimes$ 10-beta Competent $E$. coli & New England Biolabs & Cat. No. C3019 \\
\hline $\begin{array}{l}\text { VSV-SARS-CoV-2 S-glycoprotein- } \\
\text { pseudotyped virus }\end{array}$ & (Ou et al., 2020) & N/A \\
\hline $\begin{array}{l}\text { MLV-based SARS-CoV-2 S-glycoprotein- } \\
\text { pseudotyped virus }\end{array}$ & (Walls et al., 2020) & N/A \\
\hline \multicolumn{3}{|l|}{ Biological Samples } \\
\hline $\begin{array}{l}\text { Serum and plasma of SARS-CoV-2 infected } \\
\text { individuals }\end{array}$ & This paper & $\mathrm{N} / \mathrm{A}$ \\
\hline $\begin{array}{l}\text { Serum and plasma of pre-pandemic healthy } \\
\text { donors }\end{array}$ & This paper & N/A \\
\hline PBMCs of donors S2A, S2H, S2X & This paper & N/A \\
\hline \multicolumn{3}{|l|}{ Chemicals and Recombinant Proteins } \\
\hline $10 \%$ Igepal CA-630 & Sigma-Aldrich & Cat. No. 18896 \\
\hline $25 \mathrm{mM}$ dNTPs & Cytiva & Cat. No. 28406560 \\
\hline RNase OUT & Life Technologies & Cat. No. $10777-019$ \\
\hline Superscript III RT & Life Technologies & Cat. No. $18080-044$ \\
\hline DNase/RNase free water & Life Technologies & Cat. No. 10977-035 \\
\hline 10 mM dNTPs mix & Cytiva & Cat. No. 28406564 \\
\hline Q5 high fidelity DNA polymerase & Bioconcept & Cat. No. M0493S \\
\hline Agel-HF & New England Biolabs & Cat. No. R3552 \\
\hline Sall-HF & New England Biolabs & Cat. No. R3138 \\
\hline Xhol & New England Biolabs & Cat. No. R0146 \\
\hline BsiWl & New England Biolabs & Cat. No. R3553 \\
\hline Notl & New England Biolabs & Cat. No. R0189 \\
\hline Kpnl & New England Biolabs & Cat. No. R0142 \\
\hline Cutsmart & New England Biolabs & Cat. No. B7204S \\
\hline T4 DNA ligase & New England Biolabs & Cat. No. M0202 \\
\hline T4 ligase buffer & New England Biolabs & Cat. No. B0202S \\
\hline 293fectin & ThermoFisher Scientific & Cat. No. 12347019 \\
\hline
\end{tabular}




\begin{tabular}{|c|c|c|}
\hline \multicolumn{3}{|l|}{ Continued } \\
\hline REAGENT or RESOURCE & SOURCE & IDENTIFIER \\
\hline PEI MAX & Polysciences & Cat. No. POL24765-1 \\
\hline $\begin{array}{l}\text { 4-Nitrophenyl phosphate disodium salt } \\
\text { hexahydrate (pNPP) }\end{array}$ & Sigma-Aldrich & Cat. No. N2765-100TAB \\
\hline RBD mouse Fc-tagged & Sino Biological & Cat. No. $40592-\mathrm{V} 05 \mathrm{H}$ \\
\hline $\begin{array}{l}\text { Streptavidin-alkaline phosphatase } \\
\text { conjugated }\end{array}$ & Jackson ImmunoResearch & Cat. No. $016-050-084$ \\
\hline ACE2 Protein, Human & Sino Biological & Cat. No. $10108-\mathrm{H} 08 \mathrm{H}$ \\
\hline Blocker Casein (1\%) in PBS & ThermoFisher Scientific & Cat. No. 37528 \\
\hline Tween 20 & Sigma Aldrich & Cat. No. 93773 \\
\hline SARS-CoV-2 $\mathrm{S}_{2}$ sheep Fc-tagged & The Native Antigen Company & Cat. No. REC31807 \\
\hline Bovine Serum Albumine & Sigma & Cat. No. 3059 \\
\hline Trypsin, TPCK-Treated & Bioconcept & Cat. No. LS003741 \\
\hline Strep-Tactin XT & IBA GmbH & https://www.iba-lifesciences.com \\
\hline $\begin{array}{l}\text { SARS-CoV-2 Nucleoprotein, His- } \\
\text { tagged (E. coli) }\end{array}$ & The Native Antigen Company & Cat. No. REC31812-500 \\
\hline Human SARS-CoV RBD His- tagged & Sino Biological & Cat. No. 40150-V08B2 \\
\hline Sodium thiocyanate (NaSCN) & Sigma-Aldrich & Cat. No. 251410 \\
\hline BioLock Biotin blocking solution & IBA GmbH & Cat. No. 2-0205-050 \\
\hline \multicolumn{3}{|l|}{ Cell Lines } \\
\hline $\begin{array}{l}\text { ExpiCHO-S (female ovarian epithelial } \\
\text { cell line) }\end{array}$ & ThermoFisher Scientific & Cat. No. A29127 \\
\hline $\begin{array}{l}\text { HEK293T (female embryonic kidney } \\
\text { epithelial cell line) }\end{array}$ & ATCC & Cat. No. CRL-11268 \\
\hline $\begin{array}{l}\text { Expi293F and FreeStyle } 293-\mathrm{F} \text { (female } \\
\text { embryonic kidney epithelial cell line) }\end{array}$ & ThermoFisher Scientific & Cat. No. A14527; R79007 \\
\hline $\begin{array}{l}\text { CHO stably expressing WT SARS-CoV-2 S } \\
\text { (female ovarian epithelial cell line) }\end{array}$ & This study & $\mathrm{N} / \mathrm{A}$ \\
\hline \multicolumn{3}{|l|}{ Commercial Assays, Kits and Products } \\
\hline $\begin{array}{l}\text { EZ-Link }^{\mathrm{TM}} \text { NHS-PEG Solid-Phase } \\
\text { Biotinylation Kit - Mini-Spin Columns }\end{array}$ & ThermoFisher & Cat. No. 21450 \\
\hline Pierce $^{\mathrm{TM}}$ NHS-Activated Magnetic Beads & ThermoFisher & Cat. No. 88826 \\
\hline Streptavidin, Alexa Fluor ${ }^{\mathrm{TM}} 647$ conjugate & Life Technologies & Cat. No. S21374 \\
\hline $\begin{array}{l}\text { HiTrap Protein A columns (HiTrap Mab } \\
\text { select Xtra) }\end{array}$ & Cytiva & Cat. No. $28-4082-61$ \\
\hline HiTrap Fast desalting columns & Cytiva & Cat. No. 17-5087-01 \\
\hline XT Superflow ${ }^{\circledR}$ high capacity cartridge & IBA GmbH & Cat. No. 2-4026-001 \\
\hline HisTALON Superflow Cartridge, $5 \mathrm{ml}$ & Takara Bio & Cat. No. 635683 \\
\hline HisTALON Superflow Cartridge, $1 \mathrm{ml}$ & Takara Bio & Cat. No. 635650 \\
\hline Superdex 200 Increase 10/300 GL & Cytiva & Cat. No. 28-9909-44 \\
\hline Superose 6 Increase $10 / 300 \mathrm{GL}$ & Cytiva & Cat. No. 29091596 \\
\hline StrepTrap HP column, 1 ml & Cytiva & Cat. No. $28-9075-46$ \\
\hline Kifunensine & Cayman Chemical & 10009437 \\
\hline EndoH & New England BioLabs & Cat. No. P0702L \\
\hline Polyvalan Crystallophore $\mathrm{No}^{\circ} 1$ & Molecular Dimensions & Cat. No. MD2-82 \\
\hline Spectraplate-384 with high protein binding & Perkin Elmer & Cat. No. CUSG83093 \\
\hline Bio-Glo ${ }^{\mathrm{TM}}$ Luciferase Assay System & Promega AG & Cat. No. G7940 \\
\hline ExpiFectamine $^{\mathrm{TM}} \mathrm{CHO}$ Transfection Kit & Life Technologies & Cat. No. A29130 \\
\hline ExpiFectamine $^{\mathrm{TM}} 293$ Transfection Kit & Thermo Fisher Scientific & Cat. No. A14524 \\
\hline CaptureSelect CH1-XL MiniChrom column & ThermoFisher Scientific & Cat. No. 5943462005 \\
\hline
\end{tabular}




\begin{tabular}{|c|c|c|}
\hline \multicolumn{3}{|l|}{ Continued } \\
\hline REAGENT or RESOURCE & SOURCE & IDENTIFIER \\
\hline HiPrep ${ }^{\mathrm{TM}} 26 / 10$ desalting columns & Cytiva & Cat. No. $17-5087-02$ \\
\hline Zeba $^{\mathrm{TM}}$ Spin Desalting Columns & Thermo Scientific & Cat. No. 89892 \\
\hline \multicolumn{3}{|l|}{ Deposited Data } \\
\hline SARS-CoV-2 S/S2H13 (closed) & This paper & PDB-7JV6, EMD-22494 \\
\hline SARS-CoV-2 S/S2H13 (local refinement) & This paper & PDB-7JV2, EMD-22491 \\
\hline SARS-CoV-2 S/S2H13 (one RBD open) & This paper & PDB-7JV4, EMD-22492 \\
\hline SARS-CoV-2 S/S2H14 (two RBDs open) & This paper & EMD-22507 \\
\hline SARS-CoV-2 S/S2H14 (three RBDs open) & This paper & EMD-22508 \\
\hline SARS-CoV-2 S/S2A4 & This paper & PDB-7JVC, EMD-22506 \\
\hline SARS-CoV-2 S/S2A4 (local refinement) & This paper & PDB-7JVA, EMD-22497 \\
\hline SARS-CoV-2 S/S304 & This paper & PDB- 7JW0, EMD-22512 \\
\hline SARS-CoV-2 S/S2X35 & This paper & EMD-22516 \\
\hline SARS-CoV-2 S/ S2X35 (local refinement) & This paper & EMD-22517 \\
\hline $\mathrm{S} 2 \mathrm{H} 14 \mathrm{Fab}$ & This paper & PDB-7JXC \\
\hline S2A4 Fab & This paper & PDB-7JXD \\
\hline $\mathrm{S} 2 \times 35 \mathrm{Fab}$ & This paper & PDB-7JXE \\
\hline RBD/S304/S309/S2H14 & This paper & PDB-7JX3 \\
\hline \multicolumn{3}{|l|}{ Recombinant DNA } \\
\hline $\begin{array}{l}\text { Human antibody expression vectors (lgG1, } \\
\text { lgl, Igk) }\end{array}$ & Tiller et al., 2008 & N/A \\
\hline $\begin{array}{l}\text { Plasmid encoding SARS-CoV-2 2P S } \\
\text { ectodomain (amino acids 14-1211) with } \\
\text { C-terminal 8xHis-Tag }\end{array}$ & (Walls et al., 2020) & GenBank: NC_045512.2 \\
\hline $\begin{array}{l}\text { Plasmid encoding SARS-CoV-2 RBD } \\
\text { (amino acids 328-531) with C-terminal } \\
\text { 8xHis-Tag }\end{array}$ & (Walls et al., 2020) & GenBank: NC_045512.2 \\
\hline $\begin{array}{l}\text { Plasmid encoding SARS-CoV-2 RBD } \\
\text { (amino acids 328-531) with C-terminal } \\
\text { Thrombin-linker-Twin-Strep-8xHis-tag or } \\
\text { linker-Strep-8xHis-tag }\end{array}$ & This study & GenBank: NC_045512.2 \\
\hline $\begin{array}{l}\text { Plasmid encoding SARS-CoV-2 2P S } \\
\text { ectodomain (residues 14-1211) with } \\
\text { C-terminal Avi and 8xHis-Tag }\end{array}$ & This study & GenBank: NC_045512.2 \\
\hline $\begin{array}{l}\text { Plasmid encoding SARS-CoV-2 domain A } \\
\text { (amino acids 14-302) with C-terminal } \\
\text { 8xHis-Tag }\end{array}$ & This study & GenBank: NC_045512.2 \\
\hline $\begin{array}{l}\text { Plasmid encoding SARS-CoV 2P S } \\
\text { ectodomain (residues 14-1193) with } \\
\text { C-terminal 8xHis-Tag }\end{array}$ & (Walls et al., 2020) & GenBank: YP_009724390.1 \\
\hline $\begin{array}{l}\text { Plasmid encoding human ACE2 receptor } \\
\text { (amino acids 19-615) plus C-terminal } \\
\text { thrombin cleavage site, Twin-Strep- } \\
\text { 10xHis-Tag }\end{array}$ & This study & UniProt: Q9BYF1 \\
\hline phCMV1 expression vector & Genlantis & Cat. No. P003100 \\
\hline NFAT-driven luciferase gene & Promega AG & Cat. No. G9798, G7018 and G9995 \\
\hline \multicolumn{3}{|l|}{ Software and Algorithms } \\
\hline Prism 8 & GraphPad & https://www.graphpad.com/ \\
\hline UNICORN (v7.3) & Cytiva & https://www.cytivalifesciences.com/ \\
\hline OpenLAB CDS (v2.5) & Agilent Technologies & https://www.agilent.com/ \\
\hline Biacore T200 Evaluation Software (v3.1) & Cytiva & https://www.cytivalifesciences.com/ \\
\hline
\end{tabular}




\begin{tabular}{|c|c|c|}
\hline \multicolumn{3}{|l|}{ Continued } \\
\hline REAGENT or RESOURCE & SOURCE & IDENTIFIER \\
\hline Coot (v0.9) & (Emsley et al., 2010) & $\begin{array}{l}\text { https://www2.mrc-Imb.cam.ac.uk/ } \\
\text { personal/pemsley/coot/ }\end{array}$ \\
\hline MOE (v2019.0102) & Chemical Computing Group & https://www.chemcomp.com \\
\hline Refmac5 (v5.8.0258) & (Murshudov et al., 2011) & $\begin{array}{l}\text { www2.mrc-Imb.cam.ac.uk/groups/ } \\
\text { murshudov/content/refmac/refmac.html }\end{array}$ \\
\hline Phaser (v2.8.3) & (McCoy et al., 2007) & $\begin{array}{l}\text { https://www.phaser.cimr.cam.ac. } \\
\text { ukindex.php }\end{array}$ \\
\hline XDS & (Kabsch, 2010) & http://xds.mpimf-heidelberg.mpg.de/ \\
\hline Relion & (Scheres, 2012a) & N/A \\
\hline Warp & (Tegunov and Cramer, 2019) & N/A \\
\hline Cryosparc & (Punjani et al., 2017) & $\mathrm{N} / \mathrm{A}$ \\
\hline Leginon & (Suloway et al., 2005) & N/A \\
\hline NONMEM (v7.4) & $\begin{array}{l}\text { Icon Development Solutions, Hanover } \\
\text { MD, USA }\end{array}$ & $\begin{array}{l}\text { https://www.iconplc.com/innovation/ } \\
\text { nonmem/ }\end{array}$ \\
\hline R 4.0.2 & R Studio for Statistical Computing & https://rstudio.com/products/rstudio/ \\
\hline Igor Pro 8 (v8.00) & WaveMetrics, Inc. & $\begin{array}{l}\text { https://www.wavemetrics.com/software/ } \\
\text { igor-pro-8 }\end{array}$ \\
\hline MAFFT (v7.471) & (Katoh et al., 2019) & https://mafft.cbrc.jp/alignment/server/ \\
\hline EMBOSS Needle & (Needleman and Wunsch, 1970) & $\begin{array}{l}\text { https://www.ebi.ac.uk/Tools/psa/ } \\
\text { emboss_needle/ }\end{array}$ \\
\hline \multicolumn{3}{|l|}{ Instruments } \\
\hline BD FACSAria III & BD Biosciences & https://www.bdbiosciences.com/ \\
\hline ÄKTA Xpress FPLC & Cytiva & https://www.cytivalifesciences.com/ \\
\hline ÄKTA Pure 25 & Cytiva & https://www.cytivalifesciences.com/ \\
\hline Synergy H1 Hybrid Multi-Mode plate reader & Biotek & https://www.biotek.com/ \\
\hline EL406 washer/dispenser BSL2 M & Biotek & https://www.biotek.com/ \\
\hline Powerwave $340 / 96$ spectrophotometer & Biotek & https://www.biotek.com/ \\
\hline Biacore T200 & Cytiva & https://www.cytivalifesciences.com/ \\
\hline Octet Red96 & Pall ForteBio & https://www.fortebio.com/ \\
\hline ZE5 Cell Analyzer (FACS) BSL2 M & Bio-rad & https://www.bio-rad.com/ \\
\hline
\end{tabular}

\section{RESOURCE AVAILABILITY}

\section{Lead Contact}

Further information and requests for resources and reagents should be directed to and will be fulfilled by the Lead Contact, David Veesler (dveesler@uw.edu).

\section{Materials Availability}

Materials generated in this study will be made available on request and may require a material transfer agreement.

\section{Data and Code Availability}

The cryo-EM maps and atomic models have been deposited at the Electron Microscopy Data Bank and the PDB with accession codes listed in Tables S1 and S3.

\section{EXPERIMENTAL MODEL AND SUBJECT DETAILS}

\section{Cell lines}

Cell lines used in this study were obtained from ATCC (HEK293T, Vero-E6), ThermoFisher Scientific (FreeStyle 293-F, Expi293F, ExpiCHO-S cells). All cell lines used in this study (except FreeStyle 293-F and Expi293F) were routinely tested for mycoplasma and found to be mycoplasma-free. 
Sample donors and collection

Samples were obtained from 5 cohorts of SARS-CoV-2 infected individuals under study protocols approved by the local Institutional Review Boards (Canton Ticino Ethics Committee, Switzerland, the Ethical committee of Luigi Sacco Hospital, Milan, Italy, and WCG North America, Princeton, NJ, US). All donors provided written informed consent for the use of blood and blood components (such as PBMCs, sera or plasma) and were recruited at hospitals or as outpatients. Based on their availability, participants were enrolled and allocated to either single blood draws or longitudinal follow-up. Donors were categorized as symptomatic if they reported any COVID19-related symptoms (a, fever; b, respiratory distress; c, cough; d, throat pain; e, common cold; f, taste loss/smell loss; g, diarrhea; $h$, fatigue; i, muscle bone pain; j, headache). Donors from the Ticino healthcare workers cohort were further categorized based on symptom severity as follows: asymptomatic (declaration of no symptom experience), low symptomatic (1 or 2 symptoms of a-f and any of $\mathrm{g}-\mathrm{j}$ ), mild symptomatic (any 3 symptoms of a-f); high symptomatic (any 4 symptoms of a-f), severe symptomatic (any 4 symptoms of a-f, including b), atypical (all the remaining cases).

\section{METHOD DETAILS}

Isolation of peripheral blood mononuclear cells (PBMCs), plasma and sera PBMCs and plasma were isolated from blood draw performed using tubes or syringes pre-filled with heparin, followed by Ficoll density gradient centrifugation. Sera were obtained from blood collected using tubes containing clot activator, followed by centrifugation. PBMCs, plasma and sera were stored in liquid nitrogen and $-80^{\circ} \mathrm{C}$ freezers until use, respectively.

\section{Ab discovery and recombinant expression}

$\mathrm{S} 2 \mathrm{H} 13, \mathrm{~S} 2 \mathrm{H} 14$ and S2A4 mAbs were isolated from plasma cells or memory B cells, as previously described (Corti et al., 2011; Pinto et al., 2020). S2X35 was isolated from SARS-CoV-2 S-specific CD19 IgG $^{+}$B cells sorted using a C-terminal biotinylated SARS-CoV$2 \mathrm{~S}$ ectodomain trimer conjugated to Streptavidin, Alexa Fluor 647 (Life Technologies).

Recombinant mAbs were expressed as IgG1 or Fab in ExpiCHO-S cells transiently co-transfected with plasmids expressing the heavy and light chain, as previously described (Stettler et al., 2016). Recombinant Abs were affinity purified using HiTrap Protein A columns (Cytiva) followed by desalting against phosphate-buffered saline (PBS) using HiTrap Fast desalting columns (Cytiva). All liquid chromatography purification steps were performed on a ÄKTA express FPLC (Cytiva). The final products were sterilized by filtration through $0.22 \mu \mathrm{m}$ filters and stored at $4^{\circ} \mathrm{C}$.

Fabs were expressed using transient transfection of ExpiCHO-S cells with ExpiCHO expression medium and ExpiFectamine $\mathrm{CHO}$ Transfection Kit (Life Technologies), purified by affinity chromatography on ÄKTA Xpress Mab System (Cytiva) with UNICORN 5.11 software version (Build 407) using CaptureSelect CH1-XL MiniChrom columns (ThermoFisher Scientific), buffer exchanged to PBS using a HiPrep 26/10 desalting columns (Cytiva) and sterilized through a $0.22 \mu \mathrm{m}$ filter.

\section{Recombinant glycoprotein production}

The SARS-CoV-2 and SARS-CoV prefusion S ectodomain trimers were previously described (Walls et al., 2020; Walls et al., 2019). Briefly, the SARS-CoV-2 and SARS-CoV S ectodomains were synthesized by Genscript or GeneArt, respectively, with a mu-phosphatase signal peptide, 2P stabilizing mutations (Kirchdoerfer et al., 2018; Pallesen et al., 2017), a TEV cleavage site, a foldon trimerization domain, and octa-histidine tag. The SARS-CoV-2 domain A construct (residue 14-302) was synthesized by Genscript into pcDNA3.1- with an N-terminal mu-phosphatase signal peptide and a C-terminal octa-histidine tag (GSS(H)8). All constructs were produced in FreeStyle 293-F cells grown in suspension using FreeStyle 293 expression medium (Life technologies) at $37^{\circ} \mathrm{C}$ in a humidified $8 \% \mathrm{CO}_{2}$ incubator rotating at $130 \mathrm{rpm}$. The cultures were transfected using PEI $(9 \mu \mathrm{g} / \mathrm{mL})$ with cells grown to a density of 2.5 million cells per $\mathrm{mL}$ and cultivated for 3-4 days. The supernatants were harvested and cells resuspended for another 3-4 days in fresh media, yielding two harvests. Proteins were purified from clarified supernatants using a $5 \mathrm{~mL}$ Cobalt affinity column (Takara Bio), concentrated and flash frozen in a buffer containing $20 \mathrm{mM}$ Tris pH 8.0 and $150 \mathrm{mM} \mathrm{NaCl}$ prior to analysis. SDS-PAGE or negative stain EM was run to check purity.

For SPR experiments, a SARS-CoV-2 prefusion stabilized S ectodomain with an Avi-tag between the foldon domain and the 8x His-tag was codon optimized, synthesized and cloned into the phCMV1 vector by ATUM. For protein expression, Expi293F cells were transfected using ExpiFectamine according to Thermo Fisher's Expi293 expression system user guide. Supernatants were harvested after 4 days of expression and purified over a $5 \mathrm{~mL}$ Cobalt affinity column (Takara Bio). IMAC elution peak was pooled, concentrated and injected onto a Superose 6 Increase 10/300 GL size exclusion chromatography column (Cytiva) using 1x PBS $\mathrm{pH} 7.4$ as a running buffer. SEC fractions corresponding to the main protein peak were pooled, flash frozen in liquid nitrogen and stored at $-80^{\circ} \mathrm{C}$.

RBD proteins for ELISA were produced in Expi293 cells using the phCMV1 SARS-CoV-2 RBD plasmid, which encodes for an N-terminal mu-phosphatase signal peptide, an 'ETGT' linker, SARS-CoV-2 residues 328-531, a linker sequence and Strep-8xHis-tag. Supernatants were harvested five days after transfection, equilibrated with $0.1 \mathrm{M}$ Tris- $\mathrm{HCl}, 0.15 \mathrm{M} \mathrm{NaCl}, 10 \mathrm{mM}$ EDTA, pH 8.0 and supplemented with a biotin blocking solution (IBA Lifesciences). RBD was purified by affinity chromatography on a Strep-Trap HP $5 \mathrm{~mL}$ column followed by elution with $50 \mathrm{mM}$ biotin and buffer exchange into PBS. 
To produce SARS-CoV-2 RBD for crystallization, the phCMV1 SARS-CoV-2 RBD expression plasmid was used, which encodes for an N-terminal mu-phosphatase signal peptide, an 'ETGT' linker, SARS-CoV-2 residues 328-531 and a C-terminal 8xHis-tag. Protein was expressed in Expi293F cells in the presence of $10 \mu \mathrm{M}$ kifunensine at $37^{\circ} \mathrm{C}$ and $8 \% \mathrm{CO}_{2}$ in a humidified incubator. Transfection was performed using ExpiFectamine 293 reagent (Thermo Fisher Scientific) and $1 \mu \mathrm{g}$ plasmid per ml of cell culture. Cell culture supernatant was collected after three days and supplemented with EDTA-free Protease Inhibitor (Thermo Scientific Pierce) and 10X PBS to a final concentration of $2.5 x(342.5 \mathrm{mM} \mathrm{NaCl}, 6.75 \mathrm{mM} \mathrm{KCl}$ and $29.75 \mathrm{mM}$ phosphate). SARS-CoV-2 RBD was purified using a $5 \mathrm{~mL}$ HisTALON Superflow cartridge (Takara Bio) and subsequently dialyzed against $50 \mathrm{mM} \mathrm{Tris-HCl} \mathrm{pH} \mathrm{7.5,} 150 \mathrm{mM} \mathrm{NaCl}$. SARSCoV-2 RBD was deglycosylated by overnight incubation with EndoH glycosidase at $4^{\circ} \mathrm{C}$.

For SPR, SARS-CoV-2 RBD (residues 328-531) with a C-terminal thrombin cleavage site, linker, Twin-Strep, 8xHis-tag was expressed in Expi293F cells at $37^{\circ} \mathrm{C}$ and $8 \% \mathrm{CO} 2$ in a humidified incubator. Transfection was performed using ExpiFectamine 293 reagent (Thermo Fisher Scientific) and $1 \mu \mathrm{g}$ plasmid per $\mathrm{ml}$ of cell culture. The protein was purified by affinity chromatography using a $1 \mathrm{~mL}$ HisTALON Superflow cartridge as described above (Takara Bio) and subsequently buffer exchanged using a Zeba spin desalting column into Cytiva 1x HBS-N buffer.

Antibody Fab fragments for mAbs S304, S309 and S2H14 were obtained from ATUM. To form the quaternary SARS-CoV-2 RBDS304-S309-S2H14 Fab complex, the deglycosylated SARS-CoV-2 RBD was mixed with a 1.3-fold molar excess of S304 Fab, S309 $\mathrm{Fab}$ and $\mathrm{S} 2 \mathrm{H} 14 \mathrm{Fab}$. The complex was purified on a Superdex 200 Increase 10/300 GL column pre-equilibrated with $20 \mathrm{mM}$ Tris- $\mathrm{HCl}$ $\mathrm{pH} 7.5,150 \mathrm{mM} \mathrm{NaCl}$, and concentrated to $4 \mathrm{mg} / \mathrm{mL}$. $100 \mu \mathrm{l}$ of the protein solution were mixed with 0.3 mg Polyvalan Crystallophore $\mathrm{N} \times 1$ and this solution was used for setting up crystallization trays.

Recombinant ACE2 was expressed in ExpiCHO-S cells transiently transfected with a plasmid encoding ACE2 residues 19-615, a C-terminal thrombin cleavage site, Twin-Strep-tag and 10xHis-tag. Cell culture supernatant was collected nine days after transfection, supplemented with buffer to a final concentration of $80 \mathrm{mM}$ Tris- $\mathrm{HCl} \mathrm{pH} \mathrm{8.0,100} \mathrm{mM} \mathrm{NaCl}$, and then incubated with BioLock solution for one h. ACE2 was purified using a $1 \mathrm{~mL}$ StrepTrap High Performance column (Cytiva) followed by size exclusion chromatography using a Superdex 200 Increase 10/300 GL column pre-equilibrated in $20 \mathrm{mM} \mathrm{Tris-HCl} \mathrm{pH} \mathrm{7.5,} 150 \mathrm{mM} \mathrm{NaCl}$.

\section{Enzyme-linked immunosorbent assay (ELISA)}

Spectraplate-384 with high protein binding treatment (custom made from Perkin Elmer) were coated overnight at $4^{\circ} \mathrm{C}$ with $1 \mu \mathrm{g} / \mathrm{mL}$ of SARS-CoV-2 S, domain A (in-house produced), N (The Native Antigen company), S2 (The Native Antigen company), SARS-CoV S (inhouse produced) or $5 \mu \mathrm{g} / \mathrm{mL}$ of SARS-CoV-2/SARS-CoV RBD (in-house produced) in PBS, pH 7.2, and plates were subsequently blocked with Blocker Casein (1\%) in PBS (Thermo Fisher Scientific) supplemeted with 0.05\% Tween 20 (Sigma Aldrich). The coated plates were incubated with serial dilutions of human mAbs or human plasma or sera for $1 \mathrm{~h}$ at room temperature. The plates were then washed with PBS containing 0.1\% Tween-20 (PBS-T), and Alkaline Phosphatase-conjugated Goat Anti-Human IgG, IgM or IgA (Southern Biotech) were added and incubated for $1 \mathrm{~h}$. Plates were washed three times with PBS-T, and 4-NitroPhenyl Phosphate (pNPP, Sigma-Aldrich) substrate was added and incubated for $1 \mathrm{~h}$ (IgG) or $2 \mathrm{~h}$ (IgA and $\operatorname{lgM}$ ). The absorbance of $405 \mathrm{~nm}$ was measured by a microplate reader (Biotek), and the data was plotted with Graph Prism software.

In the depletion experiments, the efficiency was calculated based on the ratio of the binding titers before and after depletion and is expressed as a percentage: $\left(1-\left(E_{50}(\text { after }) / E D_{50}(\text { before })\right)^{\star} 100\right.$.

For chaotropic ELISA, after incubation with sera, plates were washed and incubated with $1 \mathrm{M}$ solution of sodium thiocyanate (NaSCN) for $1 \mathrm{~h}$. Avidity Index was calculated as the ratio (\%) between the $\mathrm{ED}_{50}$ in presence and the $\mathrm{ED}_{50}$ in absence of $\mathrm{NaSCN}$.

\section{Depletion of RBD-specific Abs from plasma or serum samples}

Magnetic beads (Pierce) were washed with ice-cold $1 \mathrm{mM} \mathrm{HCl}$ solution. $1 \mathrm{mg} / \mathrm{mL}$ RBD solution was coupled to magnetic beads in $50 \mathrm{mM}$ borate buffer, $\mathrm{pH} 8.5$, with a $2 \mathrm{~h}$ incubation at room temperature. Beads were washed 3 times with $0.1 \mathrm{M}$ glycine, $\mathrm{pH} 2.0$, followed by a wash with purified water. Beads were then incubated with quenching buffer ( $3 \mathrm{M}$ ethanolammine, $\mathrm{pH} 9.0)$ for $2 \mathrm{~h}$. Beads were washed with purified water and resuspended in storage buffer ( $50 \mathrm{mM}$ borate buffer, $\mathrm{pH} 8.5$, with $0.05 \%$ sodium azide). Serum or plasma were diluted to $1 / 50$ in $500 \mu$ PBS containing 1/20 (25 $\mu$ l) of RBD-magnetic beads and incubated for $1 \mathrm{~h}$ at room temperature with continuous rotation. Tubes were placed on a magnetic holder and supernatants were collected.

Pseudovirus neutralization assays

VSV-based SARS-CoV-2 S-glycoprotein-pseudotyped viruses were used to test the neutralizing activity of serum or plasma from COVID-19 recovered patients. Briefly, HEK293T cells were transfected with a SARS-CoV-2 S glycoprotein-encoding plasmid harboring the $\Delta 19 \mathrm{C}$-terminal truncation (Ou et al., 2020) using the X-tremeGENE HP DNA transfection reagent (Merk) according to the manufacturer's instructions and then incubated at $37^{\circ} \mathrm{C}$ with $8 \% \mathrm{CO}_{2}$ for $24 \mathrm{~h}$. Next, the transfected cells were infected with Delta-G-VSV-Luc in DMEM and incubated $1 \mathrm{~h}$ at $37^{\circ} \mathrm{C}$ with $5 \% \mathrm{CO}_{2}$. After removing the infection medium, the cells were washed twice with PBS and DMEM containing $10 \%$ FBS and $1 \%$ penicillin-streptomycin was added. Infected cells were further incubated for $24 \mathrm{~h}$ at $37^{\circ} \mathrm{C}$ before the supernatant containing the VSV-SARS-CoV-2 S pseudoviruses was collected, cleaned from cellular debris by centrifugation, and stored at $-80^{\circ} \mathrm{C}$. VSV-SARS-CoV-2 S pseudovirus was incubated with serial dilution of serum or plasma for $1 \mathrm{~h}$ in white culture 96 well plate at $37^{\circ} \mathrm{C}$. Next, VeroE6 at 20000 cells/well were added to the mix and incubated $2 \mathrm{~h}$ at $37^{\circ} \mathrm{C}$. After $2 \mathrm{~h}, \mathrm{MEM}$ supplemented with $40 \%$ FBS and $4 \%$ penicillin-streptomycin was added to the cells for additional $24 \mathrm{~h}$. Culture medium was then 
removed from the cells and $50 \mu \mathrm{L} /$ well of Bio-Glo (Promega) diluted 1:2 with PBS $\mathrm{Ca}^{2+} \mathrm{Mg}^{2+}$ was added. After 5 min incubation in the dark, the luminescence signal was measured using a Synergy $\mathrm{H} 1 \mathrm{Hybrid}$ Multi-Mode plate reader (Biotek). Measurements were performed in duplicate and relative luciferase units (RLU) were converted into neutralization percentages and plotted with a nonlinear regression curve fit in Graph Prism.

In the depletion experiments, the efficiency was calculated based on the ratio of the neutralizing titers before and after depletion and is expressed as a percentage: (1-(ID80(after)/ID80(before)) ${ }^{\star} 100$.

MLV-based SARS-CoV-2 S-pseudotyped viruses were used to test the neutralizing activity of recombinant mAbs, as previously described (Walls et al., 2020). HEK293T cells were co-transfected with a SARS-CoV-2 S glycoprotein-encoding-plasmid harboring the $\triangle 19 \mathrm{C}$-terminal truncation, an MLV Gag-Pol packaging construct and the MLV transfer vector encoding a luciferase reporter using the X-tremeGENE HP DNA transfection reagent (Merk) according to the manufacturer's instructions. VeroE6 cells were cultured in MEM containing 10\% FBS, $1 \%$ penicillin-streptomycin and plated into 96 -well plates for $16-24 \mathrm{~h}$ at 20000 cells/well. Pseudovirus, pre-activated with TPCK (Bioconcept) at $10 \mu \mathrm{g} / \mathrm{mL}$ for $1 \mathrm{~h}$ at $37^{\circ}$, with or without serial dilution of mAbs was incubated for $1 \mathrm{~h}$ and then added to the wells after washing $3 \mathrm{X}$ with MEM. A mAb of unrelated specificity was used as a negative control. After 2-3 $\mathrm{h}$ MEM containing 20\% FBS and $2 \%$ penicillin-streptomycin was added to the cells for $48 \mathrm{~h}$. Following $48 \mathrm{~h}$ of infection, culture medium was removed from the cells and $50 \mu \mathrm{l} /$ well of Bio-Glo (Promega) diluted 1:2 with PBS $\mathrm{Ca}^{2+} \mathrm{Mg}^{2+}$ was added to the cells and incubated in the dark for 5 min before reading on a Synergy H1 Hybrid Multi-Mode plate reader (Biotek). Measurements were done in duplicate and RLU values were converted to percentage of neutralization and plotted with a nonlinear regression curve fit in Graph Prism.

\section{Blockade of RBD binding to ACE2}

Unlabeled mAbs or plasma/sera were serially diluted, mixed with RBD mouse Fc-tagged antigen (Sino Biological, final concentration $20 \mathrm{ng} / \mathrm{mL}$ ) and incubated for $30 \mathrm{~min}$ at $37^{\circ} \mathrm{C}$. The mix was added for $30 \mathrm{~min}$ to ELISA 96-well plates (Corning) pre-coated overnight at $4^{\circ} \mathrm{C}$ with $2 \mu \mathrm{g} / \mathrm{mL}$ human ACE2 in PBS. Plates were washed and RBD binding was revealed using secondary goat anti-mouse lgG (Southern Biotech). After washing, pNPP substrate was added and plates were read at $405 \mathrm{~nm}$. The percentage of inhibition was calculated as follow: (1-(OD sample-OD neg ctr)/(OD pos ctr-OD neg ctr)]) $\times 100$.

\section{Blockade of mAb binding to RBD}

Human anti-RBD full IgG1 mAbs were biotinylated using the EZ-Link NHS-PEO solid phase biotinylation kit (Pierce). Labeled mAbs were tested for binding to RBD by ELISA and the optimal concentration of each mAb to achieve $80 \%$ maximal binding was determined. Unlabeled mAbs or sera/plasma were serially diluted and added to ELISA 96-well plates (Corning) pre-coated overnight at $4^{\circ} \mathrm{C}$ with $1 \mu \mathrm{g} / \mathrm{mL}$ of RBD mouse Fc-tagged antigen (Sino Biological) in PBS. After $30 \mathrm{~min}$, biotinylated anti-RBD mAbs were added at the concentration achieving $80 \%$ maximal binding and the mixture was incubated at room temperature for 230 min. Plates were washed and $\mathrm{mAb}$ binding was revealed using alkaline phosphatase-comjugated streptavidin (Jackson ImmunoResearch). After washing, pNPP substrate (Sigma-Aldrich) was added and plates were read at $405 \mathrm{~nm}$. The percentage of inhibition was calculated as follow: (1-(OD sample-OD neg ctr)/ (OD pos ctr-OD neg ctr)]) $\times 100$.

\section{Cell-surface mAb-mediated $\mathrm{S}_{1}$ shedding}

CHO cells stably expressing wild-type SARS-CoV-2 S were resuspended in wash buffer (PBS 1\% BSA, 2 mM EDTA) and treated with $10 \mu \mathrm{g} / \mathrm{mL}$ TPCK-t (Bioconcept) for $30 \mathrm{~min}$ at $37 \mathrm{C}$. Cells were washed and aliquoted (90,000 cells/well). MAbs were added to cells at $15 \mu \mathrm{g} / \mathrm{mL}$ final concentration for $180 \mathrm{~min}$ at $37^{\circ} \mathrm{C}$. Cells were collected at different time points $(5,15,30,60,120,180$ and $240 \mathrm{~min})$, washed at $+4^{\circ} \mathrm{C}$ and incubated with $1.5 \mu \mathrm{g} / \mathrm{mL}$ secondary goat anti-human IgG, Fc $\gamma$ fragment specific (Jackson ImmunoResearch) on ice for $20 \mathrm{~min}$. Cells were washed and resuspended in wash buffer and analyzed with ZE5 FACS (Bio-rad).

\section{Western blot}

The prefusion SARS-CoV-2 S ectodomain trimer was incubated alone or with S2A4, S304 or S2X35 Fabs (molar ratio 1:1.2) during $0.5,1$ or $2 \mathrm{~h}$ at room temperature. Laemmli loading buffer was added prior to boiling the samples for $5 \mathrm{~min}$ at $95 \mathrm{C}$. Samples were run on a 4\%-20\% gradient Tris-Glycine Gel (BioRad) and transferred to a PVDF membrane. Membrane was blocked in $5 \%$ milk during $45 \mathrm{~min}$ at room temperature. An anti-S $\mathrm{S}_{2}$ primary antibody (1:250 dilution) and an Alexa Fluor 680-conjugated goat anti-human secondary antibody (1:50,000; Jackson Laboratory) were used for Western-blotting. A LI-COR processor was used to develop images.

\section{Measurement of effector functions}

Determination of mAb-dependent activation of human Fc $\gamma$ RIIlla was performed using SARS CoV-2 S stable transfected CHO cells as targets, incubated with titrated concentrations of mAb and after 10 min incubated with Jurkat expressing Fc $\gamma$ RIIla receptor on their surface and stable transfected with NFAT-driven luciferase gene (Promega, Cat. Nr. G9798 and G7018) at an effector to target ratio of 6:1. Activation of human Fc $\gamma \mathrm{R}$ IIIla (F158 or V158 variants) in this bioassay results in the NFAT-mediated expression of the luciferase reporter gene. Luminescence was measured after $21 \mathrm{~h}$ of incubation at $37^{\circ} \mathrm{C}$ with $5 \% \mathrm{CO}_{2}$ with a luminometer using the Bio-Glo-TM Luciferase Assay Reagent according to the manufacturer's instructions.

Determination of mAb-dependent activation of human Fc $\gamma$ Rlla was performed using SARS CoV-2 S stable transfected CHO cells as target, incubated with titrated concentrations of $\mathrm{mAb}$ and after $10 \mathrm{~min}$ incubated with Jurkat expressing Fc $\gamma \mathrm{R}$ Ila receptor on their 


\section{CellPress}

surface and stable transfected with NFAT-driven luciferase gene (Promega, Cat. Nr. G9995) at an effector to target ratio of 5:1. Activation of human Fc $\gamma$ Rlla (H131 variant) in this bioassay results in the NFAT-mediated expression of the luciferase reporter gene. Luminescence was measured after $21 \mathrm{~h}$ of incubation at $37 \mathrm{C}$ with $5 \% \mathrm{CO}_{2}$ with a luminometer using the Bio-Glo-TM Luciferase Assay Reagent according to the manufacturer's instructions.

Complement-dependent cytotoxicity (CDC) assays were performed using SARS CoV-2 S stable transfected CHO cells as target, incubated with titrated concentrations of mAb and after 10 min incubated at a concentration of 1:24 with Low-Tox M Rabbit Complement (Cedarlane Laboratories Limited, Cat. Nr.: CL3051) previously pre-absorbed with target cells alone in excess. mAb-dependent cell killing was measured using LDH release assay (Cytotoxicity Detection Kit (LDH) (Roche; Cat. Nr.: 11644793001) after 2 h of incubation at $37^{\circ} \mathrm{C}$.

Affinity and avidity determination by surface plasmon resonance (SPR)

SPR binding measurements were performed using a Biacore T200 instrument using either anti-AviTag pAb (for capturing S proteins) or StrepTactin XT (for capturing RBDs) covalently immobilized on CM5 chips. Running buffers were either Cytiva HBS-EP+ (pH 7.4) or $20 \mathrm{mM}$ phosphate $\mathrm{pH} 5.4,150 \mathrm{mM} \mathrm{NaCl}, 0.05 \% \mathrm{P}-20$, for neutral or acidic $\mathrm{pH}$ experiments, respectively. All measurements were performed at $25^{\circ} \mathrm{C}$. Acidic $\mathrm{pH}$ experiments were run as single-cycle kinetics. mAb concentrations for all experiments were 3-fold dilution series starting from $300 \mathrm{nM}$. Capture levels for neutral pH RBD experiments were $\sim 75 \mathrm{RU}$ and within $10 \%$ of each other except for S2H13 Fab data which was collected separately (capture level of $\sim 60 \mathrm{RU}$ ) and scaled proportional to capture level to allow for comparison across datasets. Approximate capture levels for other datasets are: neutral pH Fab-S $=190 \mathrm{RU}, \mathrm{neutral} \mathrm{pH}$ lgG-S $=$ $165 \mathrm{RU}$, acidic $\mathrm{pH}$ Fab-RBD $=80 \mathrm{RU}$, acidic $\mathrm{pH}$ Fab-S $=205 \mathrm{RU}$, and acidic pH IgG-S $=205 \mathrm{RU}$. Capture levels were within $\sim 10 \%$ of each other across each neutral $\mathrm{pH}$ dataset, and within $3 \%$ of each other across each acidic pH dataset. Double reference-subtracted data were fit to a 1:1 binding model using Biacore Evaluation software, which yields an "apparent $K_{D}$ " for the $S-$ binding data because the kinetics also reflect $S$ conformational dynamics and especially for the lgG binding data where the kinetics also reflect avidity. RBD-binding data were fit with a Global Rmax. Spike-binding data for the tightly-associating S309 and S2X35 Fabs as well as all IgG measurements were fit with local Rmax, due to the S conformational changes which can affect the accessibility of epitopes across different $m A b$ concentrations (these $K_{D \text {,app }}$ are indicated to be approximate). For dissociation rates that were too slow to fit, $\mathrm{K}_{\mathrm{D} \text {,app }}$ are reported as an upper limit.

Competition experiments using biolayer interferometry (Octet)

The SARS-CoV-2 RBD was loaded for $3 \mathrm{~min}$ at $8 \mu \mathrm{g} / \mathrm{mL}$ in kinetics buffer onto anti-Penta-HIS (HIS1K) biosensors (Molecular Devices, ForteBio). Association of mAbs (full-length lgG) was performed in kinetics buffer $(0.01 \%$ endotoxin-free BSA, 0.002\% Tween-20, $0.005 \%$ NaN3 in PBS) at $15 \mu \mathrm{g} / \mathrm{mL}$ for $7 \mathrm{~min}$.

Crystallization, data collection, structure determination and analysis

Crystals of the SARS-CoV-2 RBD-S304-S309-S2H14 Fab complex were obtained at $22^{\circ} \mathrm{C}$ by sitting drop vapor diffusion. A total of $200 \mathrm{~nL}$ complex were mixed with $200 \mathrm{~nL}$ mother liquor solution containing 16.2\% (w/v) PEG 4000, $0.09 \mathrm{M}$ sodium citrate pH 6.0, $0.18 \mathrm{M}$ ammonium acetate, $0.02 \mathrm{M}$ potassium acetate, $0.01 \mathrm{M}$ MES pH 6.0 and $1.5 \%$ (v/v) Pentaerythritol ethoxylate (15/4 EO/OH).

Data were collected at the Molecular Biology Consortium beamline 4.2.2 at the Advanced Light Source synchrotron facility in Berkeley, CA. Two individual datasets from the same crystal processed with the XDS software package (Kabsch, 2010), were merged for a final dataset at $2.65 \AA$ in space group C2. The RBD-S304-S309-S2H14 Fab complex structure was solved by molecular replacement using Phaser (McCoy et al., 2007) and X-ray structures of the RBD and Fabs as search models. Several subsequent rounds of model building and refinement were performed using Coot (Emsley et al., 2010), Refmac5 (Murshudov et al., 2011), and MOE (https:// www.chemcomp.com), to arrive at a final model for the ternary complex. Using the RBD-S304-S309-S2H14 Fab complex crystal structure, the S309, S304 and S2H14 binding epitopes on the RBD protein were determined by identifying all RBD residues within a $5.0 \AA$ distance from any Fab atoms. The analysis was performed using the Molecular Operating Environment (MOE) software package from the Chemical Computing Group (https://www.chemcomp.com) and the results were manually confirmed.

Optimal crystals of S2H14, S2A4 and S2X35 Fabs were obtained by the hanging-drop vapor diffusion method with a mosquito robot at $20^{\circ} \mathrm{C}$. A total of $150 \mathrm{~nL}$ of Fabs at $20 \mathrm{mg}$ per mL (for S2H14 and S2A4) or at $12 \mathrm{mg}$ per mL (for S2X35) in $20 \mathrm{mM} \mathrm{Tris-HCl} \mathrm{pH} 8.0$, $150 \mathrm{mM} \mathrm{NaCl}$ were mixed with 150nL mother liquor solution containing $0.2 \mathrm{M}$ magnesium acetate and $20 \%$ (w/v) PEG 3350 (for $\mathrm{S} 2 \mathrm{H} 14 \mathrm{Fab}$ ), $1.2 \mathrm{M}$ Ammonium sulfate, 0.1M Sodium cacodylate/Hydrochloric acid pH 6,5 (for S2A4 Fab) or $0.16 \mathrm{M} \mathrm{MgCl}_{2}, 0.08 \mathrm{M}$ Tris- $\mathrm{HCl}, \mathrm{pH} 8.5,24 \%$ (w/v) PEG 4000 and 20\% (v/v) glycerol (for S2X35 Fab). Drops were equilibrated against reservoir solutions for 1-2 weeks at room temperature after which crystals were flash cooled in liquid nitrogen using the mother liquor solution supplemented with $30 \%$ glycerol as a cryoprotectant. Diffraction data were remotely recorded on synchrotron beamline 5.0.2 at ALS, indexed and scaled using Mosfilm (Battye et al., 2011) and SCALA or aimless (Evans and Murshudov, 2013). Initial phases were obtained by molecular replacement in Phaser (McCoy et al., 2007) on the CCP4 suite, using homology models. Refinement was performed in iterations of manual model building in Coot (Emsley et al., 2010) and automatic refinement in Phenix (Liebschner et al., 2019). 
CryoEM sample preparation, data collection and data processing

$\mathrm{S} 2 \mathrm{H} 13 \mathrm{Fab}$ was generated by digestion of the corresponding monoclonal IgG with LysC (Thermo Fisher Scientific) at 1:2000 (w/w) ratio during $5 \mathrm{~h}$ at $37^{\circ} \mathrm{C}$ while Fabs S2A4, S2H14, S304 and S2X35 were recombinantly expressed as described above. SARS-CoV$2 \mathrm{~S}$ at $1.2 \mathrm{mg} / \mathrm{mL}$ was incubated with 1.2 molar excess of Fabs at $4^{\circ} \mathrm{C}$ at least for $1 \mathrm{~h}$. Three $\mu \mathrm{L}$ of $1-1.5 \mathrm{mg}$ per $\mathrm{mL}$ of complexes was loaded onto a freshly glow discharged 1.2/1.3 UltrAuFoil grid (300 mesh) prior to plunge freezing using a vitrobot MarkIV (ThermoFisher Scientific) with a blot force of 0 and $6-7.5 \mathrm{~s}$ blot time at $100 \%$ humidity and $21^{\circ} \mathrm{C}$.

Data were acquired on an FEI Titan Krios transmission electron microscope operated at $300 \mathrm{kV}$ and equipped with a Gatan K2 Summit direct detector and Gatan Quantum GIF energy filter, operated in zero-loss mode with a slit width of 20 eV. Automated data collection was carried out using Leginon (Suloway et al., 2005) at a nominal magnification of 130,000x with a super-resolution pixel size of $0.525 \AA$ and stage tilt angles up to 45 (Tan et al., 2017). The dose rate was adjusted to 8 counts/pixel/s, and each movie was fractionated in 50 frames of $200 \mathrm{~ms}$. For the S/S304 dataset, 2,791 micrographs were collected with a defocus range comprised between -1.5 and $-2.8 \mu \mathrm{m}$. For the $\mathrm{S} / \mathrm{S} 2 \mathrm{H} 13$ dataset, 6,697 micrographs were collected with a defocus range comprised between -0.4 and $-3.4 \mu \mathrm{m}$. For the S2A4 dataset, 1,995 micrographs were collected with a defocus range comprised between -0.4 and $-2.4 \mu \mathrm{m}$. Movie frame alignment, estimation of the microscope contrast-transfer function parameters, particle picking and extraction were carried out using Warp (Tegunov and Cramer, 2019). Particle images were extracted with a box size of 800 binned to 400 yielding a pixel size of $1.05 \AA$.

For the S/S2H14 and S/S2X35 complexes, data were acquired on a FEI Glacios transmission electron microscope operated at 200 kV. Automated data collection was carried out using Leginon (Suloway et al., 2005) at a nominal magnification of 36,000x with a pixel size of $1.16 \AA$ and stage tilt angle of 40 or 30 for S/S2H14 or S/S2X35 complexes, respectively (Tan et al., 2017). The dose rate was adjusted to 8 counts/pixel/s, and each movie was acquired in counting mode fractionated in 50 frames of $200 \mathrm{~ms}$. For the S/S2H14 complex, 1,886 micrographs were collected with a defocus range comprised between -1.0 and $-2.5 \mu \mathrm{m}$. For the S/S2X35 complex, 946 micrographs were collected with a defocus range comprised between -0.8 and $-2.5 \mu \mathrm{m}$. Warp (Tegunov and Cramer, 2019) was also used for movie frame alignment, estimation of the microscope contrast-transfer function parameters, particle picking and extraction.

For the S/S2H13, S/S2H14, S/S2A4 and S/S304 datasets, two rounds of reference-free 2D classification were performed using cryoSPARC (Punjani et al., 2017) to select well-defined particle images (only one round of reference-free 2D classification was performed for S/S2X35). Subsequently, two rounds of 3D classification with 50 iterations each (angular sampling 7.5 for 25 iterations and $1.8 \mathrm{p}$ with local search for 25 iterations), using our previously reported closed SARS-CoV-2 S structure as initial model (PDB 6VXX) or ab initio generated models for S2H14, S304 or S2X35 (Punjani et al., 2017), were carried out using Relion (Scheres, 2012b) without imposing symmetry. 3D refinements were carried out using non-uniform refinement along with per-particle defocus refinement in cryoSPARC (Punjani et al., 2019). Particle images were subjected to Bayesian polishing using Relion (Zivanov et al., 2018; Zivanov et al., 2019) before performing another round of non-uniform refinement in cryoSPARC followed by per-particle defocus refinement and again non-uniform refinement.

To further improve the density of the S2H13, S2A4 and S2X35 Fabs, the particles were symmetry-expanded and subjected to focus $3 D$ classification without refining angles and shifts using a soft mask encompassing the RBM and S2H13 variable domains or RBD and S2A4 or S2X35 variable domains using a tau value of 60 (S2H13 and S2A4) or 40 (S2X35). Particles belonging to classes with the best resolved Fab density were selected (all particles were retained for S2X35) and subjected to local refinement using cryoSPARC (Punjani et al., 2017; Punjani et al., 2019). Local resolution estimation, filtering, and sharpening were carried out using CryoSPARC (Cardone et al., 2013). Reported resolutions are based on the gold-standard Fourier shell correlation (FSC) of $0.143 \mathrm{criterion}$ and Fourier shell correlation curves were corrected for the effects of soft masking by high-resolution noise substitution (Chen et al., 2013).

CryoEM model building and analysis

UCSF Chimera (Pettersen et al., 2004) and Coot (Emsley et al., 2010) were used to fit atomic models (PDB 6VXX or PDB 6VYB) into the cryoEM maps and the Fab variable domains were manually built or the co-crystal structures of SARS-CoV-2 RBD with S304/S309/ S2H14 was used. Models were refined and relaxed using Rosetta using both sharpened and unsharpened maps (Frenz et al., 2019; Wang et al., 2016) and validated using Molprobity (Chen et al., 2010), Phenix (Liebschner et al., 2019), Privateer (Agirre et al., 2015) and EMRinger (Barad et al., 2015). Figures were generated using UCSF ChimeraX (Goddard et al., 2018).

\section{Longitudinal Mixed-Effects Modeling}

A non-linear mixed effects model was used to estimate parameters describing the kinetics of RBD, S- and N-specific lgG in individuals with longitudinal data following the onset of disease symptoms. Briefly, a one compartment direct response model with $1^{\text {st }}$ order input and $1^{\text {st }}$ order output was developed to describe the Ab response formation and decay. Individual parameters are assumed to be log-normally distributed and proportional residual error was employed in the modeling. Influence of gender, age and disease severity (hospitalized versus symptomatic) in Ab response formation and decay were evaluated. The analyses were conducted using NONMEM, version 7.4 (ICON Development Solutions, Hanover, MD, USA). Graphical data presentations were conducted using R 4.0.2 (R Foundation for Statistical Computing). 


\section{CellPress}

\section{QUANTIFICATION AND STATISTICAL ANALYSIS}

Statistical analyses were performed using GraphPad Prism (v8) and Microsoft Excel for Windows 10 (v16.0.13001.20254). Statistical differences were analyzed with Mann-Whitney U-test. Nonparametric Kruskal-Wallis test was used to perform multiple comparisons between groups analyzed. Correction for multiple comparison was performed with Dunn's test. Nonparametric Spearman correlation was used to compute correlations between pairs of data. Statistical significance was defined as $p<0.05$. ED $D_{50}, I_{50}$, ID80 and BD80 values were determined by non-linear regression analysis (log(agonist) versus response - Variable slope (four parameters)). 
A

\begin{tabular}{cr}
\hline Patients demographics & \\
\hline Participants & 647 \\
\hline Female & 411 \\
Male & 229 \\
N/A & 7 \\
\hline Average age & 42 \\
Range & $18-95$ \\
\hline Positive PCR for SARS-CoV-2 & 271 \\
\hline Average days post-symptom onset & 42 \\
Range & $10-147$ \\
\hline Hospitalized & 47 \\
Clinica Luganese Moncucco & 31 \\
Luigi Sacco Hospital & 16 \\
Symptomatic & 556 \\
Clinica Luganese Moncucco & 9 \\
Luigi Sacco Hospital & 1 \\
US - San Francisco & 100 \\
Ticino healthcare workers & 423 \\
Swiss volunteers & 23 \\
Asymptomatic & 44 \\
Luigi Sacco Hospital & 4 \\
US - San Francisco & 2 \\
Ticino healthcare workers & 36 \\
Swiss volunteers & 2 \\
\hline Hospitalized & 368 \\
Symptomatic & 11 \\
Asymptomatic & 329 \\
\hline Subjects with multiple time points & 28 \\
\hline
\end{tabular}

B

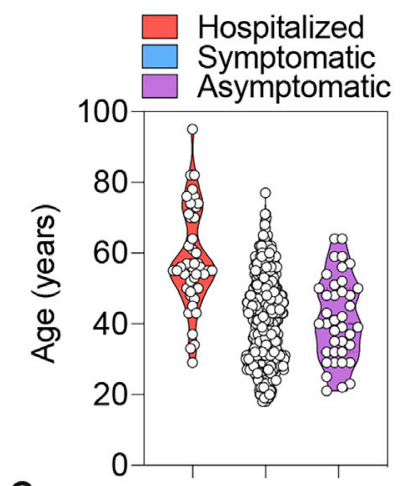

C

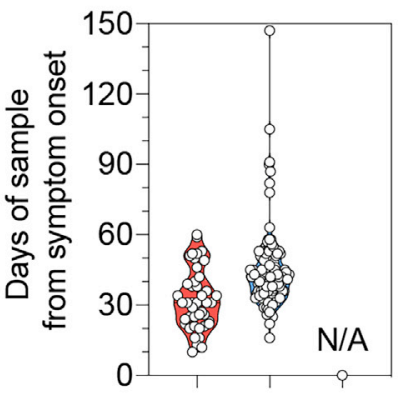

Figure S1. Description of the Cohorts of SARS-CoV-2-Infected Individuals, Related to Figures 1 and 2 (A) Summary of patient demographics.

(B) Age distribution of hospitalized, symptomatic and asymptomatic individuals.

(C) Time interval between the date of sample collection and the date of symptom onset. 
A

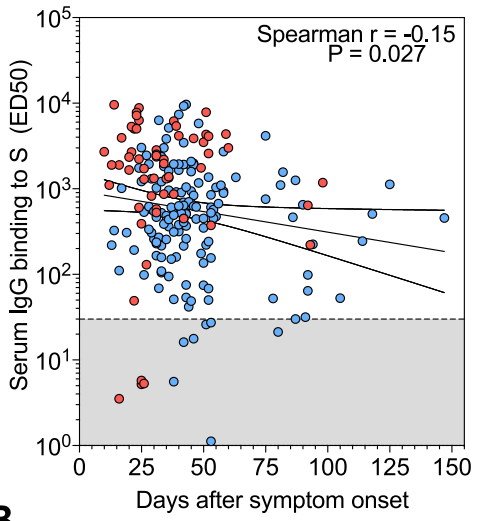

B

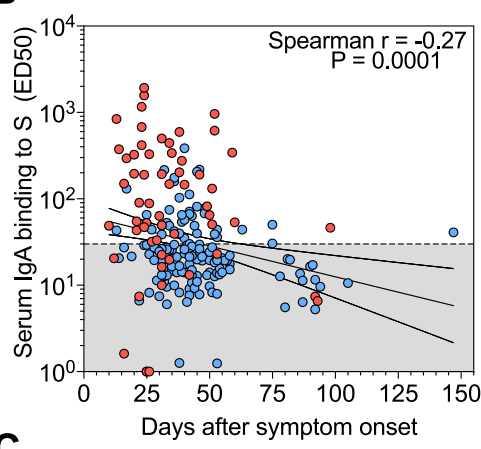

C

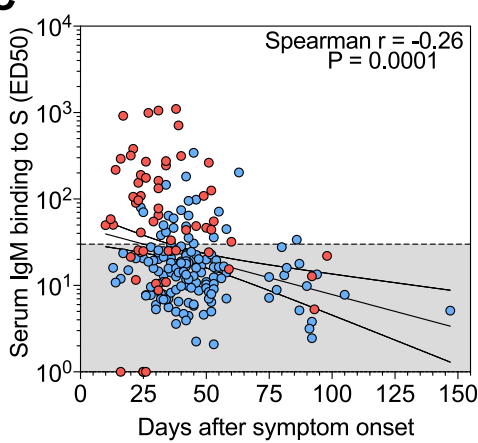

D

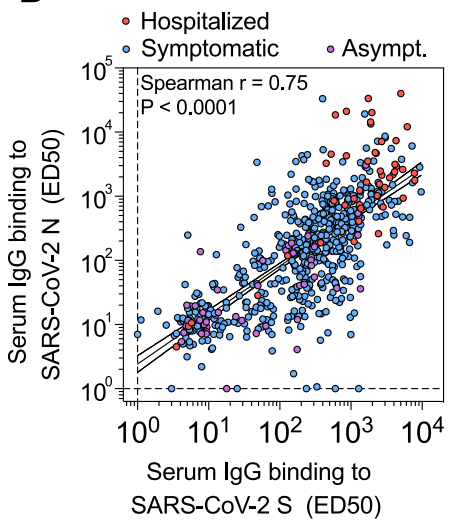

E
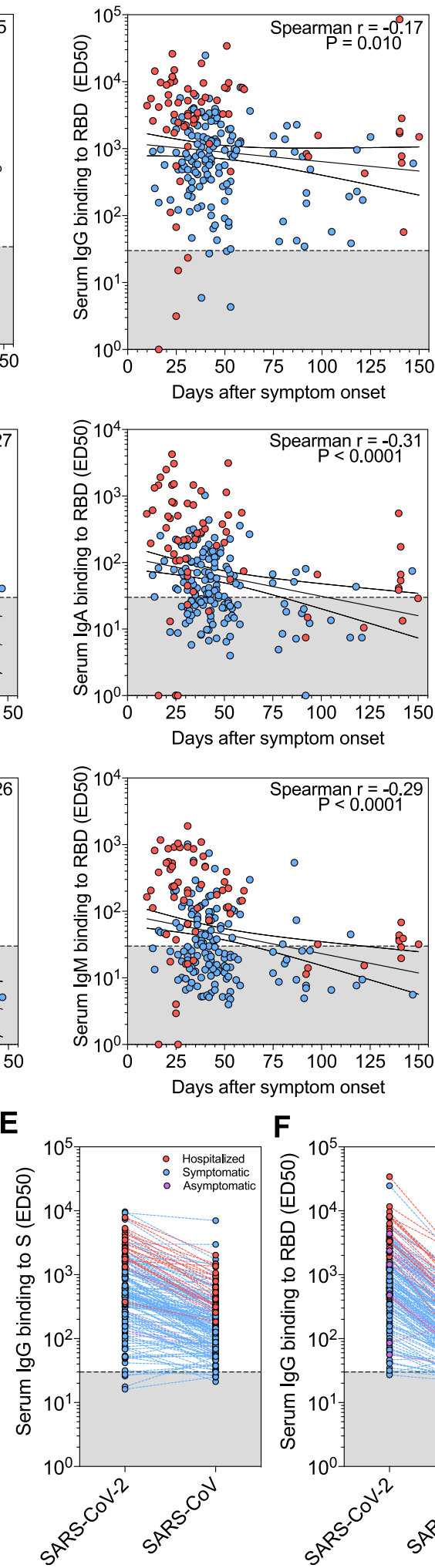
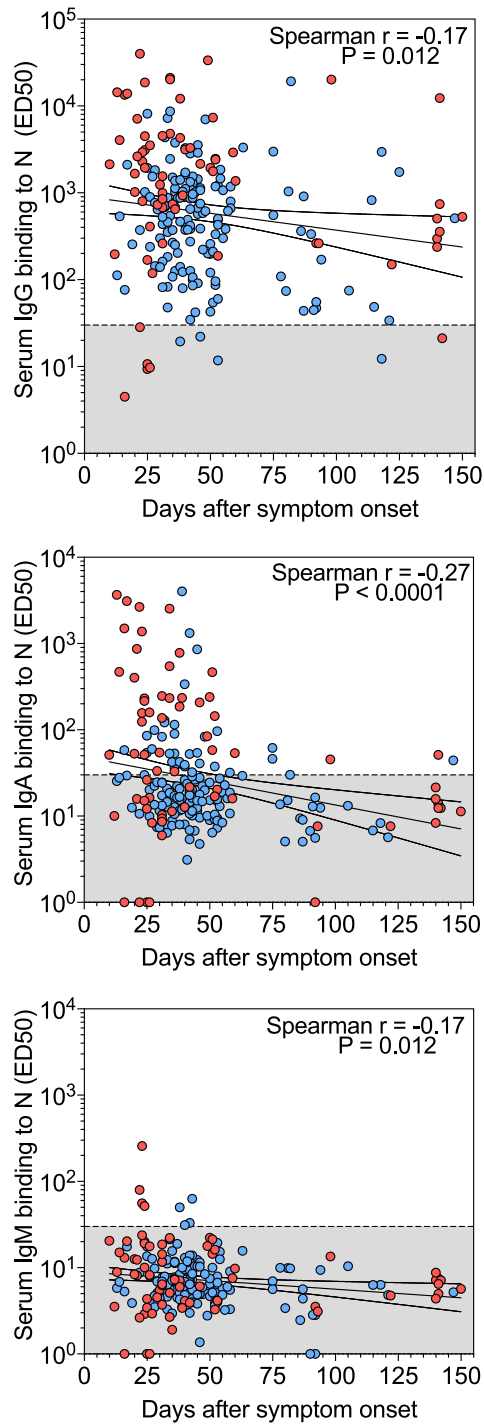

G

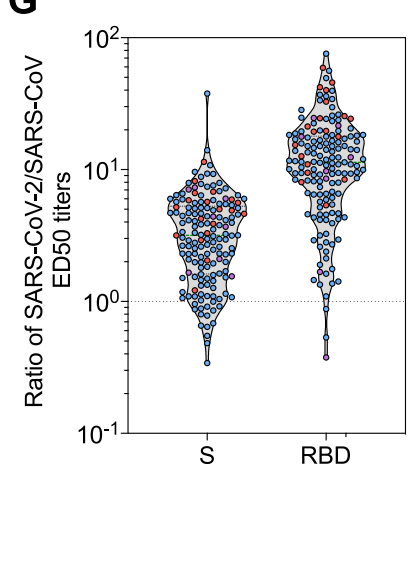


(D) Correlation between SARS-CoV-2 S- and N-specific IgG binding titers $\left(\mathrm{ED}_{50}\right)$.

( $E$ and F) IgG binding titers to SARS-CoV-2 and SARS-CoV S (E) and RBD (F) from 19 hospitalized, 130 symptomatic and 8 asymptomatic individuals.

(G) Ratios of SARS-CoV-2/SARS-CoV S and RBD IgG binding titers. 
A

\begin{tabular}{|l|l|l|l|l|l|}
\hline mAb & $\begin{array}{l}\text { VH (\% Identlty nts; } \\
\text { aa differences) }\end{array}$ & $\begin{array}{l}\text { VL (\% Identlty nts; } \\
\text { aa differences) }\end{array}$ & Source & $\begin{array}{l}\text { TIme polnt from } \\
\text { disease onset }\end{array}$ & RBD site \\
\hline S2H14 & VH3-15 $(100 ; 0)$ & VL6-57 $(100 ; 0)$ & Plasma cells & 17 days & la \\
\hline IC50, ng/mI)
\end{tabular}

B
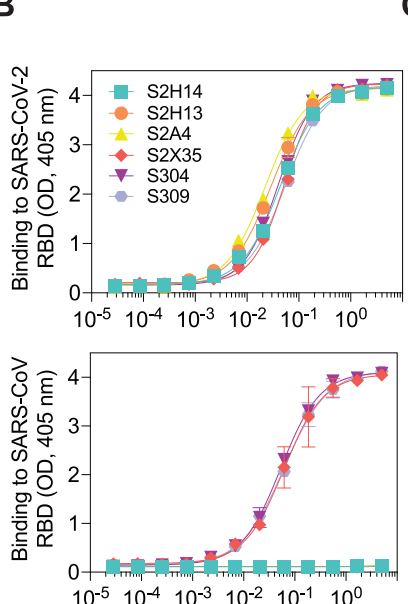

mAb concentration $(\mu \mathrm{g} / \mathrm{ml})$

F

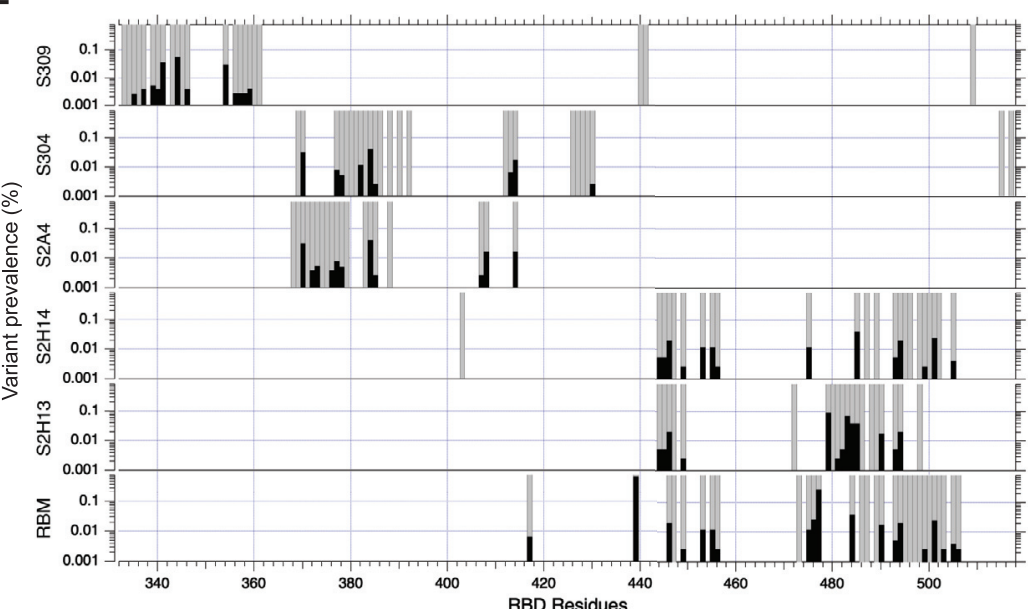

H

C

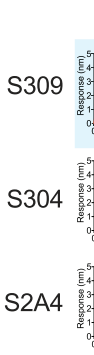

S309

$\mathrm{S} 304$

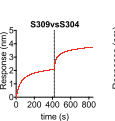

$\mathrm{S} 2 \mathrm{~A} 4$

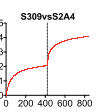

$\mathrm{S} 2 \times 35$

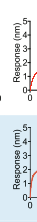
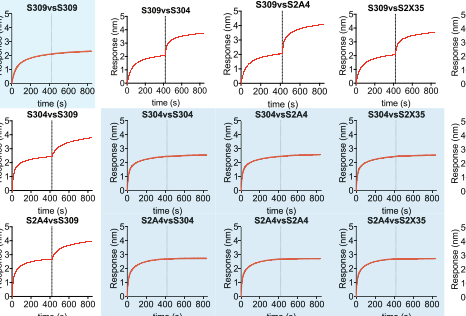

$\mathrm{S} 2 \mathrm{H} 14$

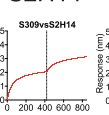

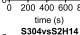
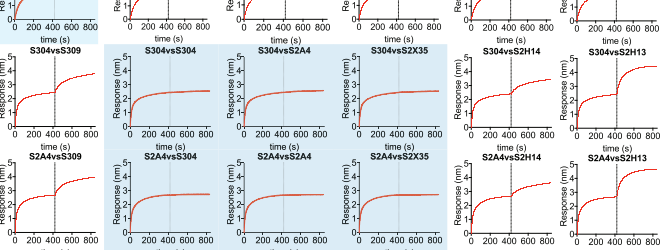

$\mathrm{S} 2 \times 35$
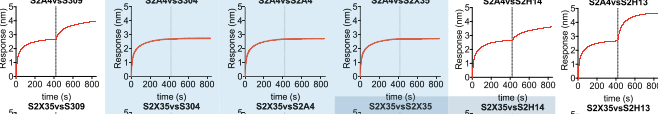

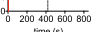

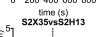

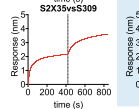

$\mathrm{S} 2 \mathrm{H} 14$

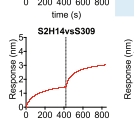

$\mathrm{S} 2 \mathrm{H} 13$
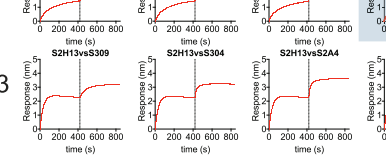

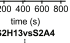
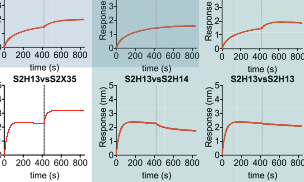

G

Incubation time (h) $\frac{\mathrm{S} 2 \mathrm{~A} 4}{0.5 \quad 1 \quad 2} \frac{\mathrm{S} 304}{0.5 \quad 12} \frac{\mathrm{S} 2 \times 35}{0.5 \quad 12} \quad \frac{\mathrm{noFab}}{0.5 \quad 12}$
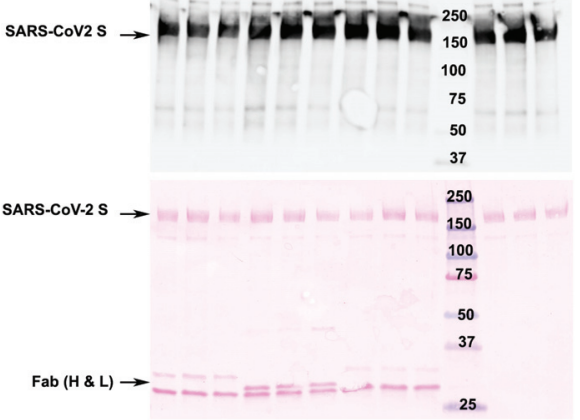

$\operatorname{Fab}(\mathrm{H} \& \mathrm{~L}) \rightarrow-\square===--{ }_{25}$

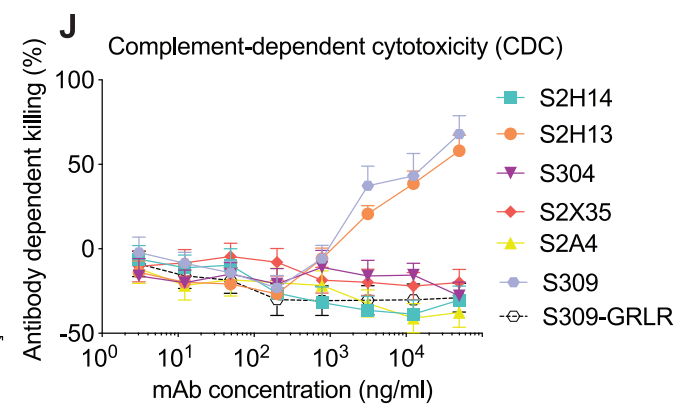

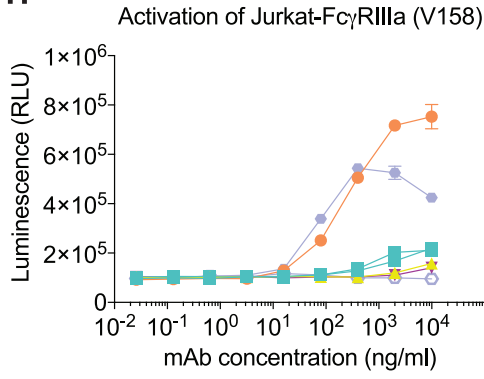

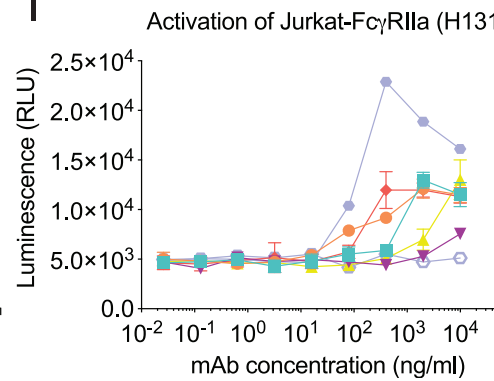

D
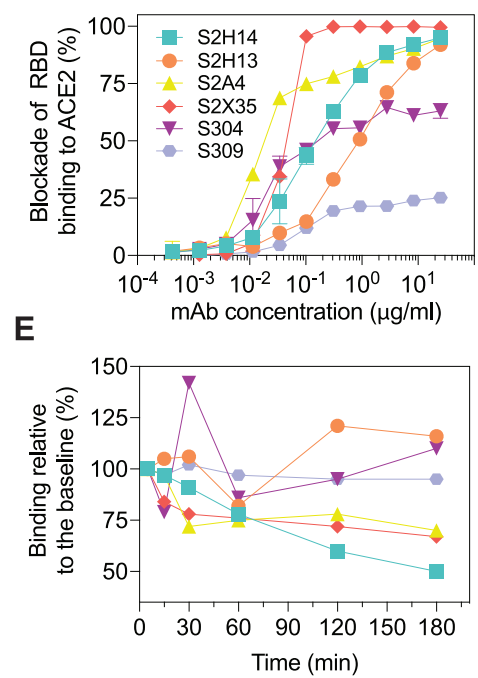
Figure S3. Characteristics of the Six Probe mAbs Used for Structural and Epitope-Mapping Studies, Related to Figures 3, 4, 5, 6, and 7 (A) V(D)J usage, percentage identity to germline, number of somatic mutations, source and time interval between sample collection and mAb isolation, RBD site recognized and neutralization potency of the 6 mAbs. B mem, memory B cell; PC, plasma cells.

(B) Binding of the 6 mAbs to the SARS-CoV-2 (up) or SARS-CoV (down) RBD analyzed by ELISA.

(C) Competition matrix for binding of each of the six mAbs in presence of another mAb evaluated by biolayer interferometry.

(D) mAb-mediated inhibition of RBD binding to ACE2 analyzed by ELISA.

(E) $m A b$-mediated $\mathrm{S}_{1}$ subunit shedding from cell-surface expressed SARS-CoV-2 $\mathrm{S}$ as determined by flow-cytometry.

(F) Conservation of RBM and epitope residues in $\sim 74,000$ SARS-CoV-2 sequences (GISAID, August $11^{\text {th }}, 2020$ ). RBM and epitope residues are shown as gray bars. Black bars indicate variant prevalence for epitope residues with at least 2 variants. RBM residues were determined from PDB $6 \mathrm{MOJ}$ using a $5.0 \AA$ distance cutoff between RBD and ACE2 residues using MOE.

(G) Western-blot analysis (top) of the prefusion-stabilized SARS-CoV-2 S ectodomain trimer in presence of S2A4, S304 or S2X35 Fab after incubation for the indicated amount of times. Red ponceau staining (bottom) of the SDS-PAGE gel used for carrying out the western blot confirming the presence of added Fabs when indicated.

(H) Analysis of activation of Fc $\gamma$ RIlla (V158 allele) expressed on Jurkat cells by SARS-CoV-2 S stably transfected CHO cells incubated with mAbs. GRLR indicates an antibody Fc variant carrying mutations that abolish binding to Fc $\gamma$ Rs.

(I) Analysis of activation of Fc $\gamma$ Rlla (H131 allele), expressed on Jurkat cells by SARS-CoV-2 S stably transfected CHO cells incubated with mAbs. (J) Killing of SARS-CoV-2 S stably transfected $\mathrm{CHO}$ cells by mAbs in the presence of complement (CDC assay). 

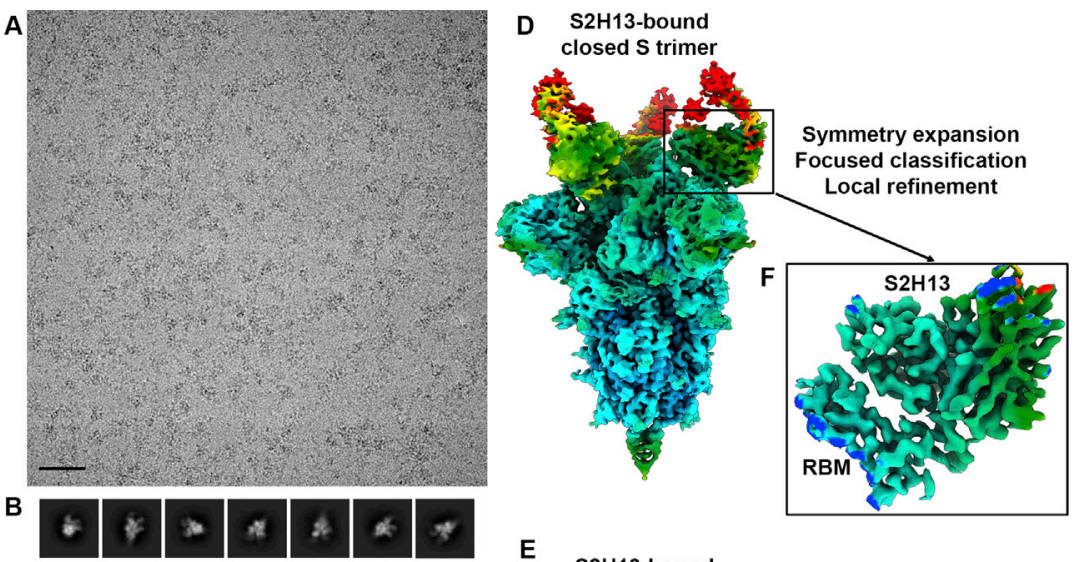

\section{E}

$\mathrm{S} 2 \mathrm{H} 13-$ bound

$S$ trimer with one RBD open
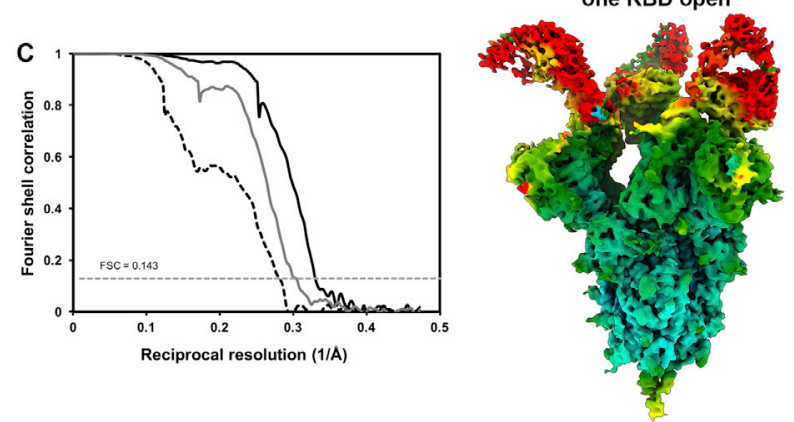

Local resolution

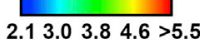

G

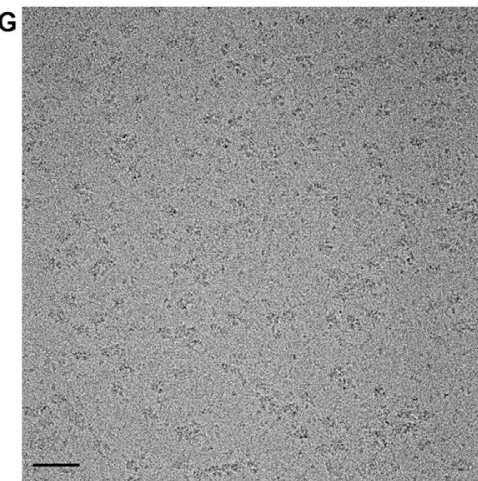

J S2H14-bound $\mathrm{S}$ trimer with one RBD closed

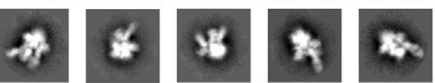

K S2H14-bound
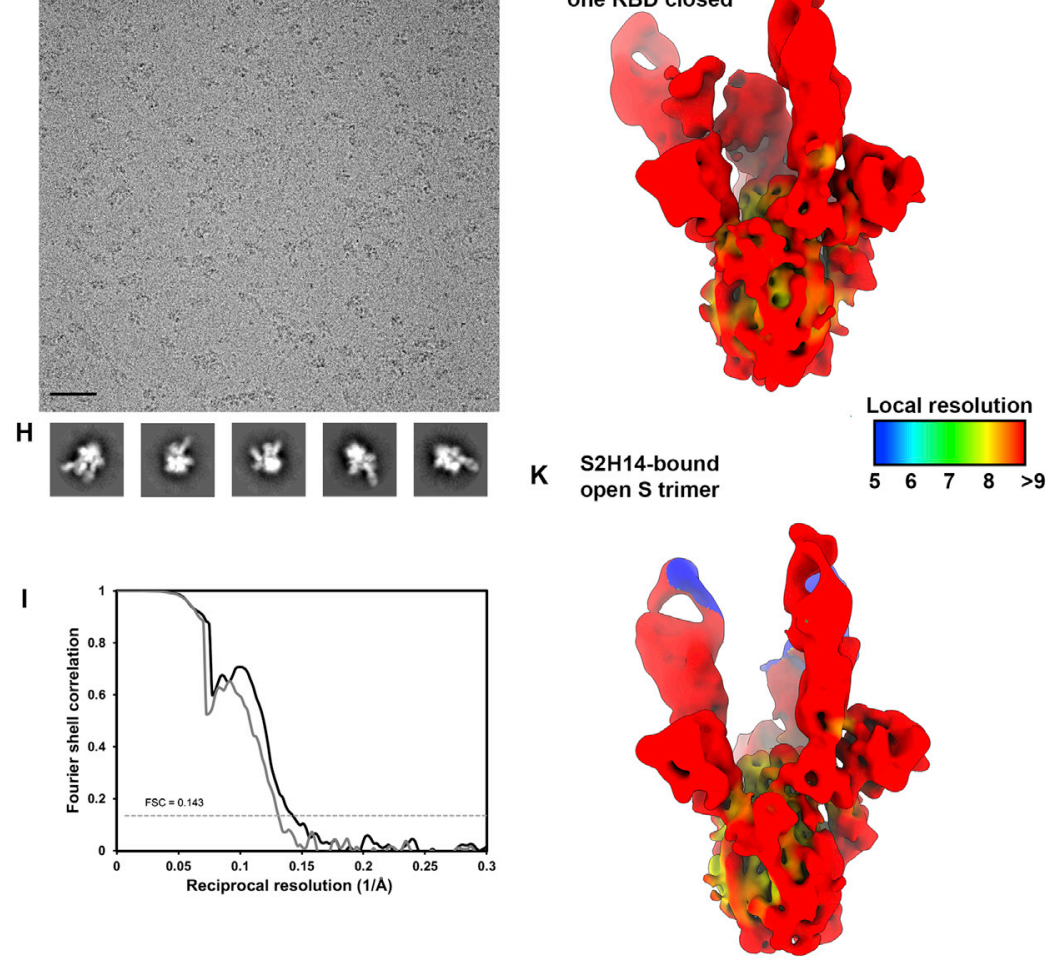
Figure S4. Cryo-EM Data Processing and Validation of the S/S2H13 and S/S2H14 Complex Datasets, Related to Figures 3 and 4 (A and B) Representative electron micrograph (A) and class averages (B) of SARS-CoV-2 S in complex with the S2H13 Fab embedded in vitreous ice. Scale bar: $400 \AA$.

(C) Gold-standard Fourier shell correlation curves for the closed S2H13-bound trimer (black solid line), partially open S2H13-bound trimer (gray solid line) and locally refined RBM/S2H13 variable domains (black dashed line). The 0.143 cutoff is indicated by horizontal dashed lines.

(D and F) Local resolution maps calculated using cryoSPARC for the closed (D) and partially open (E) reconstructions as well as for the locally refined RBM/S2H13 variable domains $(\mathrm{F})$.

$(G$ and $H)$ Representative electron micrograph $(G)$ and class averages $(H)$ of SARS-CoV-2 S in complex with the S2H14 Fab embedded in vitreous ice. Scale bar: $400 \AA$.

(I) Gold-standard Fourier shell correlation curves for the S2H14-bound trimer with one RBD closed (black solid line) or three RBDs open (gray solid line). The 0.143 cutoff is indicated by horizontal dashed lines.

( $\mathrm{J}$ and K) Local resolution maps calculated using cryoSPARC for the reconstructions with one RBD closed $(\mathrm{J})$ and three RBDs open (K). 
A mAbs binding closed RBD Abs binding open RBD

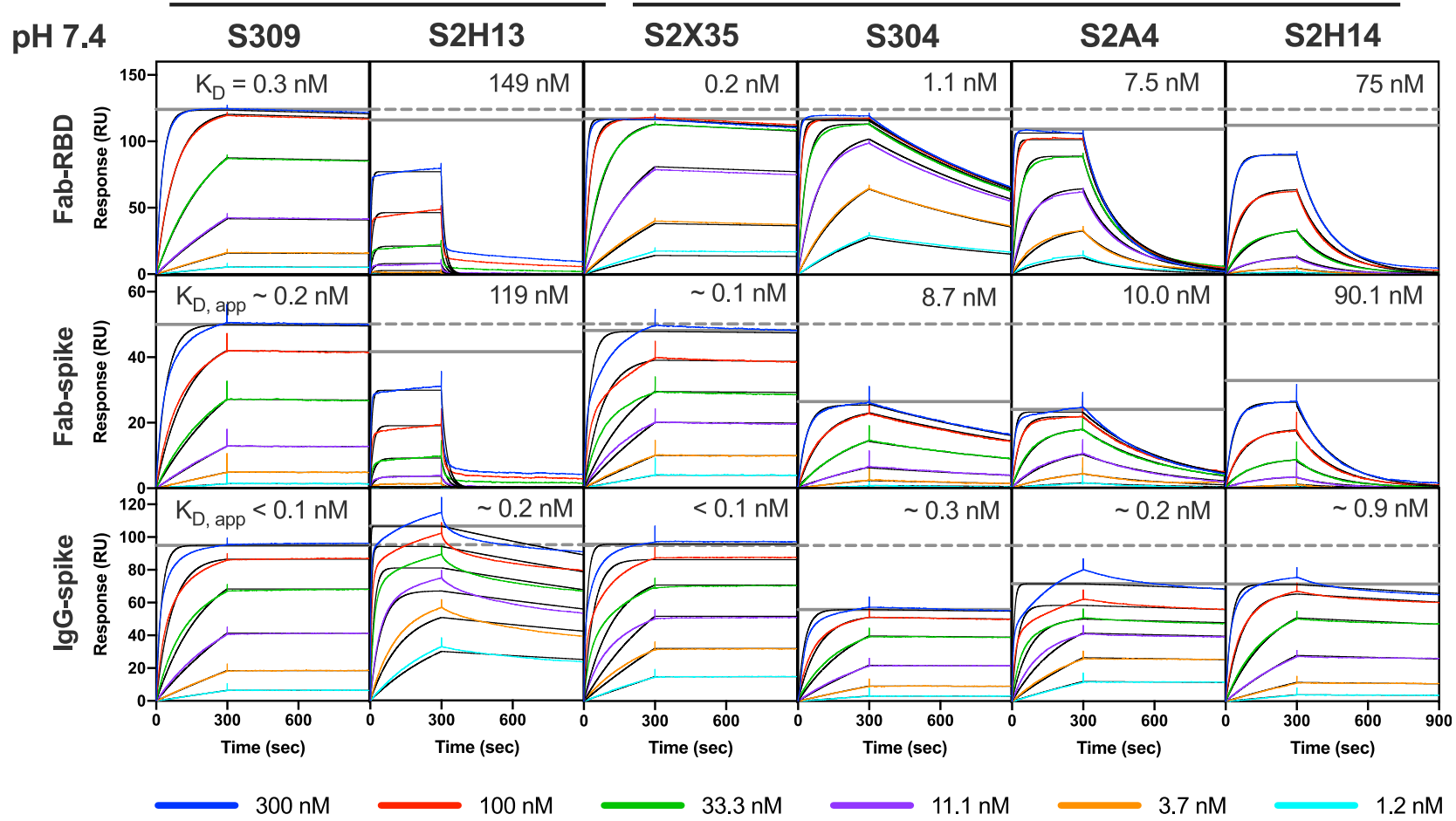

B

Fit to 1:1 Binding Model

Rmax from fit $\quad-$ S309 Rmax from fit

\section{pH 5.4}

S309

$\mathrm{S} 2 \mathrm{H} 13$

$\mathrm{S} 2 \times 35$

5304

S2A4

$\mathrm{S} 2 \mathrm{H} 14$
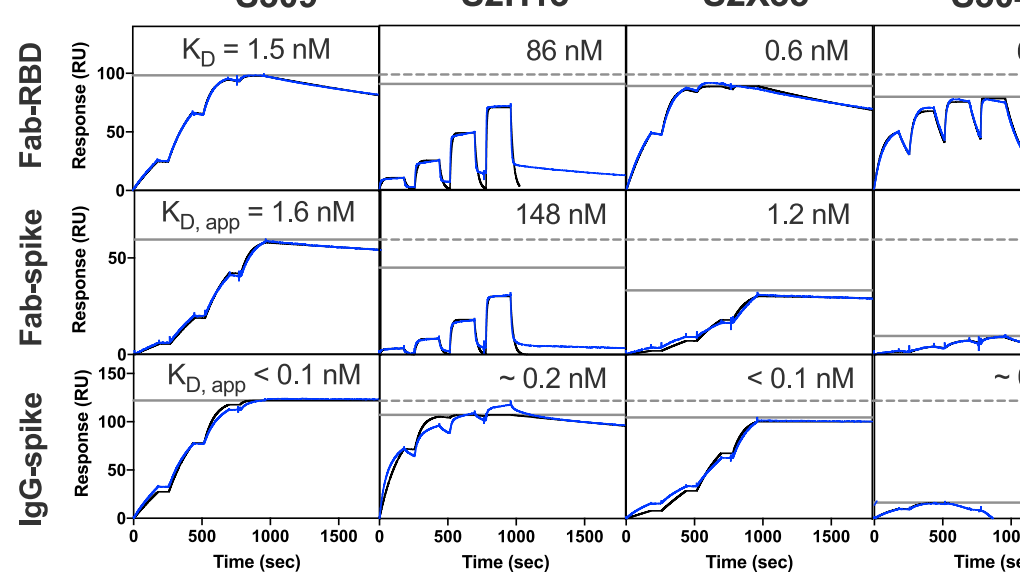

$6.0 \mathrm{nM}$

$\sqrt{\square}$

$244 \mathrm{nM}$

$[\mathrm{Ab}$

Ab] $300 \mathrm{nM}$

\begin{tabular}{c|c|c|c|}
$25 \mathrm{nM}$ & $\sqrt{ }$ & No binding & No binding
\end{tabular}

Figure S5. Analysis of Fab and IgG Binding to the Prefusion SARS-CoV-2 S Ectodomain Trimer and Recombinant RBD at Neutral and Acidic pH Analyzed by Surface Plasmon Resonance, Related to Figures 3, 4, 5, 6, and 7

(A and B) SARS-CoV-2 S or RBD was captured on the sensor chip surface and binding at multiple mAb concentrations was measured. Neutral pH measurements were performed in multi-cycle format $(\mathrm{A})$ and acidic $\mathrm{pH}$ measurements in single-cycle format (B). All data have been fit to a 1:1 binding model, which is an approximation for the S-binding data, since the kinetics incorporate conformational dynamics between open and closed RBD states, and because lgG binding involves avidity. The solid gray horizontal line gives the predicted maximum signal (saturation) based on each fit; the dashed line shows the S309 maximum binding for comparison. Asterisk indicates where a high concentration of S304 IgG was binding to the reference surface (fit was to the first two concentrations only). All mAbs bind similarly to the RBD at both pHs, but the mAbs that bind to only open RBD show a maximum below S309 in the context of the S trimer. This difference is dramatic at acidic pH where RBDs are primarily in the closed state (Zhou et al., 2020b). S2X35 was an exception, likely because its very slow off rate allows it to bias the $\mathrm{S}$ equilibrium toward open RBD. 
A

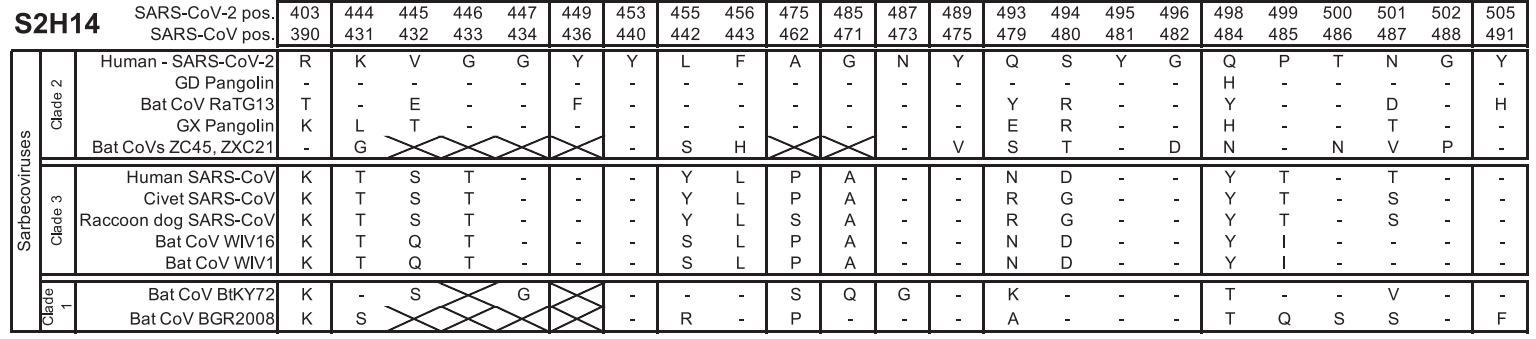

\begin{tabular}{|c|c|c|c|c|c|c|c|c|c|c|c|c|c|c|c|c|c|c|c|c|c|c|}
\hline \multicolumn{2}{|c|}{ S2H13 } & $\begin{array}{l}\text { SARS-CoV- } 2 \text { pos. } \\
\text { SARS-CoV pos. }\end{array}$ & $\begin{array}{l}444 \\
431\end{array}$ & $\begin{array}{l}445 \\
432\end{array}$ & $\begin{array}{l}446 \\
433\end{array}$ & $\begin{array}{l}447 \\
434\end{array}$ & $\begin{array}{l}449 \\
436\end{array}$ & $\begin{array}{l}472 \\
459\end{array}$ & $\begin{array}{l}479 \\
466\end{array}$ & $\begin{array}{l}480 \\
467\end{array}$ & $\begin{array}{l}481 \\
468\end{array}$ & $\begin{array}{l}482 \\
469\end{array}$ & 483 & $\begin{array}{l}484 \\
470\end{array}$ & $\begin{array}{l}485 \\
471\end{array}$ & $\begin{array}{l}486 \\
472\end{array}$ & $\begin{array}{l}488 \\
474\end{array}$ & $\begin{array}{l}489 \\
475\end{array}$ & $\begin{array}{l}490 \\
476\end{array}$ & $\begin{array}{l}493 \\
479\end{array}$ & $\begin{array}{l}494 \\
480\end{array}$ & $\begin{array}{l}498 \\
484\end{array}$ \\
\hline \multirow{11}{*}{ 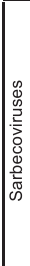 } & & Human-SARS-CoV-2 & $\mathrm{K}$ & V & G & G & $\mathrm{Y}$ & I & $\mathrm{P}$ & C & $\mathrm{N}$ & G & V & $\bar{E}$ & G & $\mathrm{F}$ & C & Y & $\mathrm{F}$ & Q & $\mathrm{s}$ & Q \\
\hline & $N$ & GD Pangolin & - & - & - & - & - & - & - & - & - & - & - & - & - & - & - & - & - & - & - & $\mathrm{H}$ \\
\hline & $\frac{\pi}{\pi}$ & Bat CoV RaTG13 & - & $\mathrm{E}$ & - & - & $\mathrm{F}$ & - & - & - & - & - & Q & T & - & $\mathrm{L}$ & - & - & Y & Y & $\mathrm{R}$ & $\mathrm{Y}$ \\
\hline & 更 & GXPangolin & L & T & & & & - & - & & - & 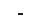 & Q & $\mathrm{v}$ & & $\mathrm{L}$ & - & - & $\mathrm{Y}$ & $\mathrm{E}$ & $\mathrm{R}$ & $\mathrm{H}$ \\
\hline & & Bat CoVs ZC45, ZXC21 & G & & & & & $\mathrm{E}$ & & & $\mathrm{N}^{*}$ & $\mathrm{G}^{*}$ & $\mathrm{~V}^{*}$ & & 6 & & $\mathrm{G}$ & $\mathrm{V}$ & $\mathrm{R}$ & $\mathrm{s}$ & $T$ & $\mathrm{~N}$ \\
\hline & & Human SARS-CoV & T & $\mathrm{s}$ & T & - & - & $\mathrm{P}$ & - & - & T & $P$ & & $\mathrm{P}$ & A & $\mathrm{L}$ & - & - & W & $\mathrm{N}$ & $\mathrm{D}$ & $\mathrm{Y}$ \\
\hline & m & Civet SARS-CoV & T & s & $\mathrm{T}$ & - & - & $\mathrm{P}$ & - & - & T & $\mathrm{P}$ & & $P$ & A & $\mathrm{P}$ & - & - & W & $\mathrm{R}$ & G & $\mathrm{Y}$ \\
\hline & 龸 & Raccoon dog SARS-CoV & T & $\mathrm{s}$ & $\mathrm{T}$ & - & - & $\mathrm{P}$ & - & - & T & $P$ & & $\mathrm{P}$ & A & $P$ & - & - & w & $\mathrm{R}$ & G & $\mathrm{Y}$ \\
\hline & & Bat CoV WIV16 & $\mathrm{T}$ & Q & $\mathrm{T}$ & - & - & $\mathrm{P}$ & - & - & T & $\mathrm{P}$ & & $\mathrm{P}$ & A & - & - & - & W & $\mathrm{N}$ & D & Y \\
\hline & & Bat CoV WIV1 & $\mathrm{T}$ & Q & $\mathrm{T}$ & - & - & $\mathrm{P}$ & - & - & $\mathrm{T}$ & $P$ & & $\mathrm{P}$ & A & - & - & - & W & $\mathrm{N}$ & D & Y \\
\hline & ¿ & Bat CoV BtKY72 & & & & & & $\mathrm{L}$ & T & - & $s$ & $\mathrm{~s}$ & & $\mathrm{~s}$ & Q & $\mathrm{L}$ & - & - & $\mathrm{E}$ & $\mathrm{K}$ & - & $\mathrm{T}$ \\
\hline & & Bat CoV BGR2008 & $\mathrm{s}$ & & & & & $\mathrm{L}$ & $\mathrm{T}$ & - & $\mathrm{s}$ & A & & - & - & L & - & - & $\mathrm{K}$ & A & - & $T$ \\
\hline
\end{tabular}

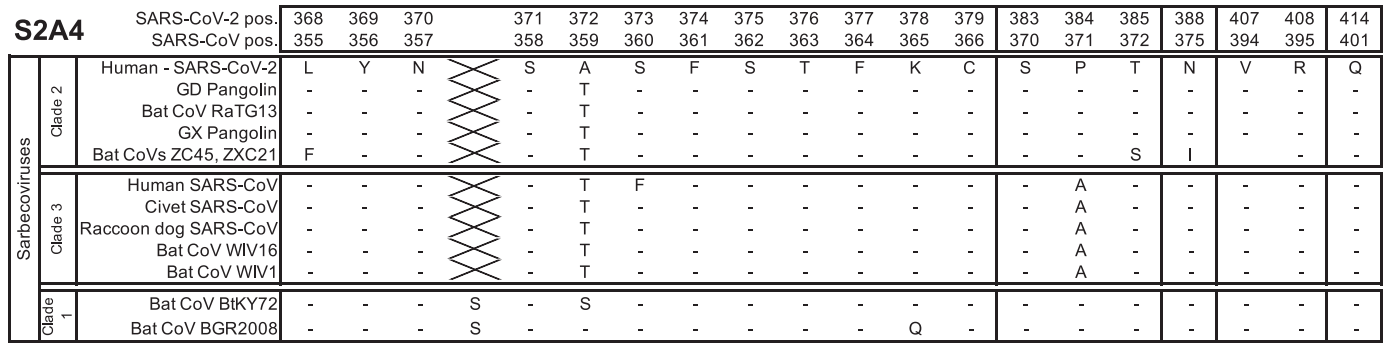

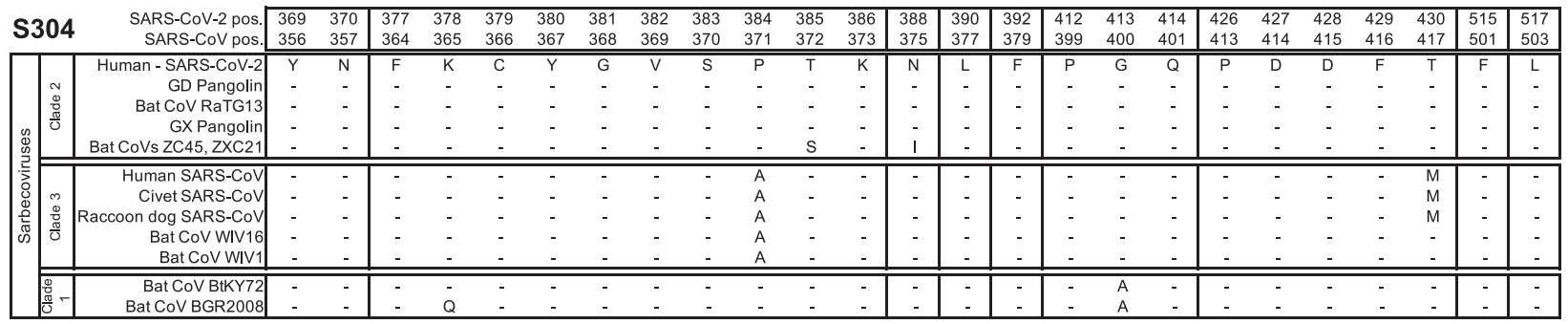

B

\begin{tabular}{|l|c|c|c|c|c|c|c|c|}
\hline & Spike & RBD & RBM & S309 & S304 & S2H13 & S2H14 & S2A4 \\
\hline $\begin{array}{l}\text { Bat CoV } \\
\text { BtKY72 } \\
\text { (Clade 1) }\end{array}$ & $72 / 84 \%$ & $74 / 85 \%$ & $46 / 54 \%$ & $62 / 86 \%$ & $96 / 96 \%$ & $30 / 50 \%$ & $57 / 70 \%$ & $95 / 100 \%$ \\
\hline $\begin{array}{l}\text { Bat CoV } \\
\text { RaTG13 } \\
\text { (Clade 2) }\end{array}$ & $97 / 98 \%$ & $90 / 94 \%$ & $67 / 83 \%$ & $86 / 95 \%$ & $100 / 100 \%$ & $55 / 65 \%$ & $65 / 78 \%$ & $95 / 95 \%$ \\
\hline $\begin{array}{l}\text { SARS-CoV } \\
\text { (Clade 3) }\end{array}$ & $76 / 87 \%$ & $74 / 82 \%$ & $42 / 50 \%$ & $81 / 95 \%$ & $92 / 92 \%$ & $30 / 35 \%$ & $44 / 48 \%$ & $84 / 84 \%$ \\
\hline
\end{tabular}

Figure S6. Conservation Analysis across Clades of Sarbecoviruses, Related to Figures 3, 4, 5, and 6 (A) S glycoprotein residues making contact with $\mathrm{S} 304, \mathrm{~S} 2 \mathrm{H} 13, \mathrm{~S} 2 \mathrm{H} 14$ or S2A4 across sarbecovirus clades. Residue numbers for both SARS-CoV-2 S and SARSCoV S are shown. Multiple sequence alignment was performed using MAFFT. A dash represents the same residue, a strikethrough represents a gap. Asterisk ( $\left.{ }^{*}\right)$ indicates manually aligned residues. Civet SARS-CoV is SARS-CoV HC/SZ/61/03 and raccoon dog SARS-CoV is SARS-CoV A031G.

(B) Identity and similarity of SARS-CoV-2 S, RBD, RBM and mAb epitopes across select sequences of the 3 sarbecovirus clades. Values were calculated using EMBOSS Needle. The insertion in the S2A4 epitope for the Clade 1 sarbecoviruses was not included in the calculation. 

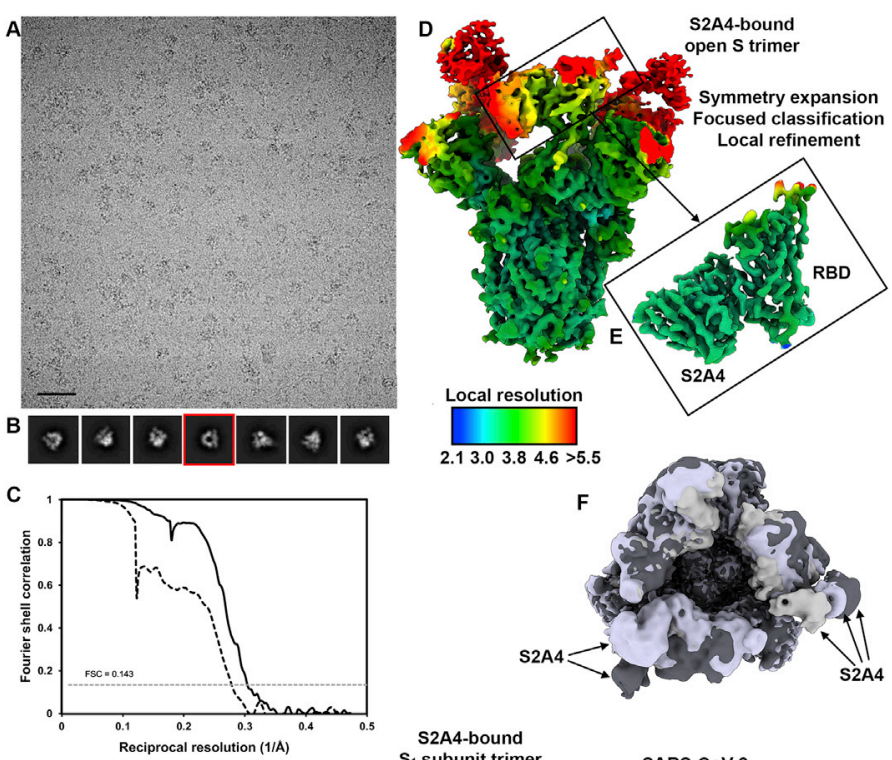

G

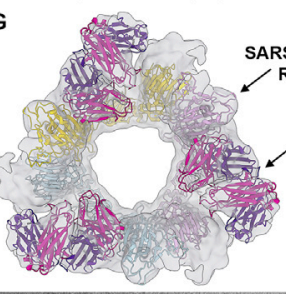

S2A4-bound

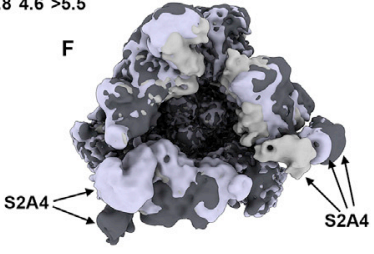

-2
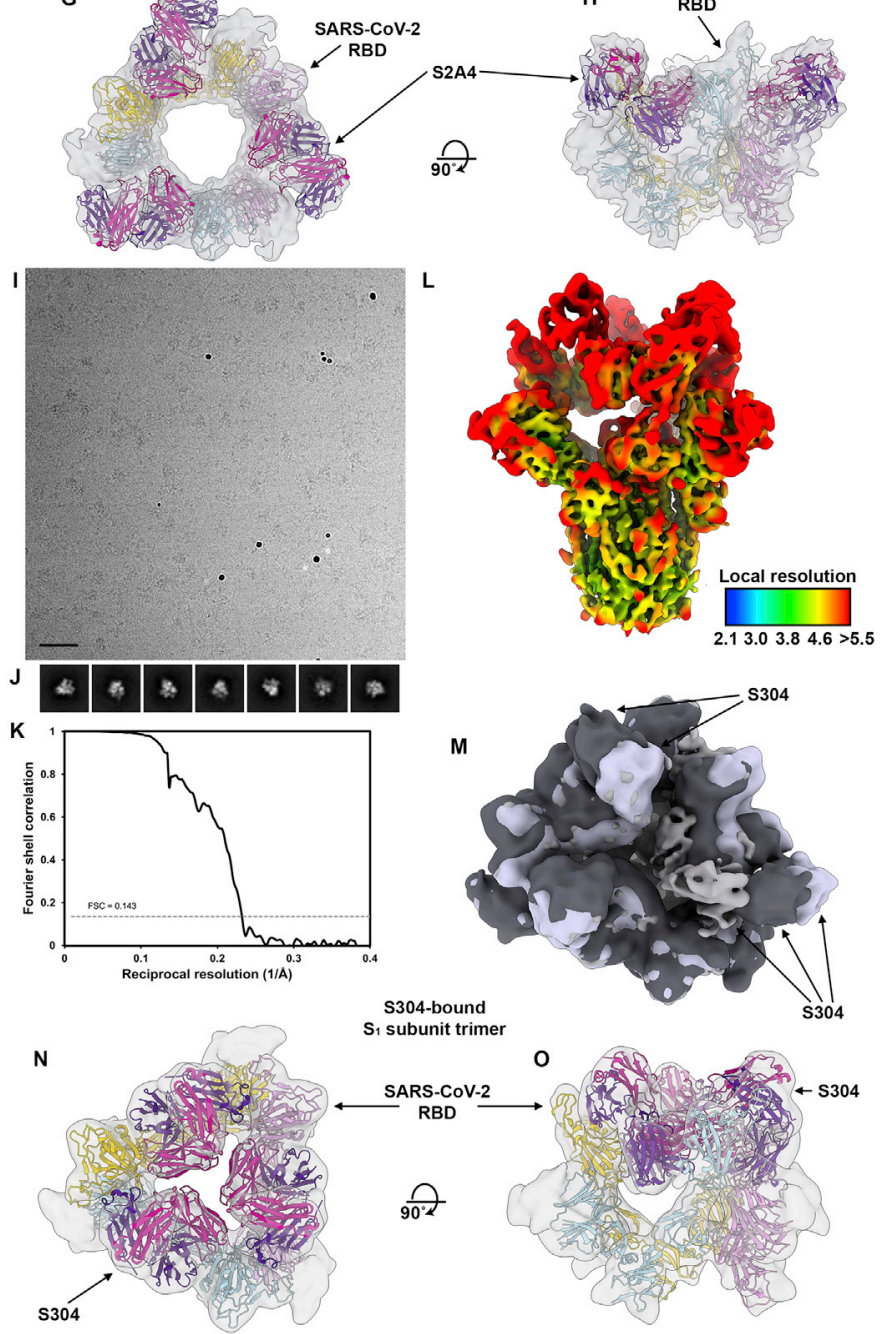
Figure S7. Cryo-EM Data Processing and Validation of the S/S2A4 and S/S304 Complex Datasets, Related to Figures 5 and 6 (A and B) Representative electron micrograph (A) and class averages (B) of SARS-CoV-2 S in complex with the S2A4 Fab embedded in vitreous ice. Scale bar: $400 \AA$. . A 2D class average corresponding to an $\mathrm{S}_{1}$ subunit trimer (with disordered $\mathrm{S}_{2}$ ) bound to three S2A4 Fabs is highlighted in red.

(C) Gold-standard Fourier shell correlation curves for the S2A4-bound trimer (black solid line) and locally refined RBD/S2A4 variable domains (black dashed line). The 0.143 cutoff is indicated by a horizontal dashed line.

( $D$ and E) Local resolution maps calculated using cryoSPARC for the whole reconstruction (D) as well as for the locally refined RBD/S2A4 variable domains (E). (F) Superimposition of the three distinct open conformations of the $S$ trimer, with three bound S2A4 Fabs and RBDs swung out to various extent. The arrows indicate the distinct positions of the Fabs in the maps.

$\left(\mathrm{G}\right.$ and $\mathrm{H}$ ) CryoEM reconstruction of the $\mathrm{S}_{1}$ subunit trimer (with disordered $\mathrm{S}_{2}$ ) bound to three S2A4 Fabs viewed along two orthogonal orientations and the corresponding atomic model fit in density. Each SARS-CoV-2 $S_{1}$ protomer is colored distinctly (cyan, pink and gold). The S2A4 light and heavy chains are colored magenta and purple, respectively.

(I and J) Representative electron micrograph (I) and class averages (J) of SARS-CoV-2 S in complex with the S304 Fab embedded in vitreous ice. Scale bar: $400 \AA$ (K) Gold-standard Fourier shell correlation curve for the S304-bound S trimer reconstruction. The 0.143 cutoff is indicated by a horizontal dashed line.

(L) Local resolution map calculated using cryoSPARC.

(M) Superimposition of the three distinct open conformations of the S trimer, with three bound S304 Fabs and RBDs swung out to various extent. The arrows indicate the distinct positions of the Fabs in the maps.

( $\mathrm{N}$ and $\mathrm{O}$ ) CryoEM reconstruction of the $\mathrm{S}_{1}$ subunit trimer (with disordered $\mathrm{S}_{2}$ ) bound to three $\mathrm{S} 304$ Fabs viewed along two orthogonal orientations and the corresponding atomic model fit in density. Each SARS-CoV-2 $\mathrm{S}_{1}$ protomer is colored distinctly (cyan, pink and gold). The S304 light and heavy chains are colored magenta and purple, respectively. 